University of Texas at El Paso

\title{
DigitalCommons@UTEP
}

Open Access Theses \& Dissertations

2014-01-01

\section{Evaluation of Static and Dynamic Postural Control in Athletes with Concussions During Recovery}

Nicholas Murray

University of Texas at El Paso, ngmurray@utep.edu

Follow this and additional works at: https://digitalcommons.utep.edu/open_etd

Part of the Biomechanics Commons

\section{Recommended Citation}

Murray, Nicholas, "Evaluation of Static and Dynamic Postural Control in Athletes with Concussions During Recovery" (2014). Open Access Theses \& Dissertations. 1308.

https://digitalcommons.utep.edu/open_etd/1308

This is brought to you for free and open access by DigitalCommons@UTEP. It has been accepted for inclusion in Open Access Theses \& Dissertations by an authorized administrator of DigitalCommons@UTEP. For more information, please contact lweber@utep.edu. 


\title{
EVALUATION OF STATIC AND DYNAMIC POSTURAL CONTROL IN ATHLETES WITH CONCUSSIONS DURING RECOVERY
}

\author{
NICHOLAS GERALD MURRAY \\ Interdisciplinary Health Sciences
}

APPROVED:

Anthony P. Salvatore, Ph.D., CCC-SLP, Chair

Rebecca J. Reed-Jones, Ph.D.

Deborah Diaz, PT, Ph.D.

Sandor Dorgo, Ph.D.

Douglas Powell, Ph.D.

Bess Sirmon-Taylor, Ph.D.

Interim Dean of the Graduate School 


\section{Copyright (C)}

by

Nicholas Gerald Murray

2014 


\section{DEDICATION}

To my wife, daughter, and family: Thank you for your patience and love during this challenging time. Without you all, I would be lost. 


\title{
EVALUATION OF STATIC AND DYNAMIC POSTURAL CONTROL IN ATHLETES WITH CONCUSSIONS DURING RECOVERY
}

\author{
by \\ NICHOLAS GERALD MURRAY, BS, MA
}

\author{
DISSERTATION \\ Presented to the Faculty of the Graduate School of \\ The University of Texas at El Paso \\ in Partial Fulfillment \\ of the Requirements \\ for the Degree of
}

DOCTOR OF PHILOSOPHY

Interdisciplinary Health Sciences Doctoral Program

THE UNIVERSITY OF TEXAS AT EL PASO

August 2014 


\section{ACKNOWLEDGEMENTS}

Through the high and low points of my ten year career in higher education, my savior walked with me every step of the way. With abounding grace and love from the Lord and my family, this portion of the journey is completed.

Thank you to each member of my committee for your time and efforts working with me to successfully complete this process. Special thanks to Dr. Reed-Jones and to Dr. Salvatore for investing in me and bringing me to the pinnacle of my academic career as a student. Without your patience and direction I would not be the individual I am today.

Lastly, to my loving wife and daughter who supported me during this difficult time. You are my joy and everything I do is for you both. Thank you for running this race called life with me. 


\begin{abstract}
Approximately seventy-six percent of individuals with a sport-related concussion present with reduced balance. Current Return-to-Play (RTP) protocols rely heavily on measures of static balance that are not environmentally relevant. However, due to the variable nature of symptom presentation, research has not clearly defined a requisite time for balance recovery following a sport-related concussion. The use of novel procedures such as monitoring postural control during an environmentally relevant dynamic balance tasks could refine the timeline for recovery of postural control and aid in mitigating the potential severe consequences of Second Impact Syndrome. Therefore, the purpose of this study was to investigate changes in postural control during a traditional quiet stance balance task and an environmentally relevant dynamic balance task during one week of recovery in athletes with concussions. Eleven collegiate athletes with concussions and twenty healthy collegiate athletes completed a static balance assessment and an experimental environmentally relevant balance assessment, the WiiFit Soccer Heading Game, during a one week of recovery period from a sport-related concussion. Peak CoP Velocity in the anteroposterior (A/P) and mediolateral (M/L) directions were calculated during quiet stance (eyes open and eyes closed) and the WiiFit Soccer Heading Game. Mixed model ANOVAs (2 groups x 2 assessment periods) ascertained significant differences in the quiet stance eyes closed $\mathrm{A} / \mathrm{P}$ direction, and in the environmentally relevant balance task in the $\mathrm{A} / \mathrm{P}$ and $\mathrm{M} / \mathrm{L}$ directions between the concussion and healthy athletes during eight days of recovery. As such, athletes with concussions are more unstable when compared to healthy athletes 24-48 hours post-injury and up to eight days of recovery. In addition, the WiiFit Soccer Heading game is a game-like balance assessment that is more challenging and more sensitive in observing balance impairment in athletes with concussions over time when compared to traditional measures. Further research is needed to ascertain if and when dynamic balance recovers alongside traditional static measures.
\end{abstract}




\section{TABLE OF CONTENTS}

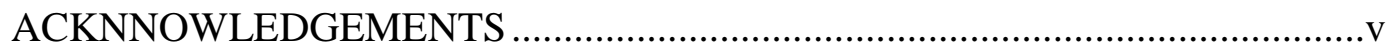

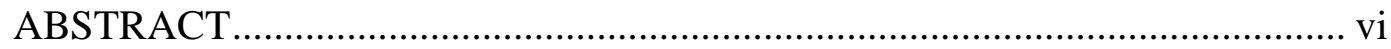

LIST OF TABLES ........................................................................... ix

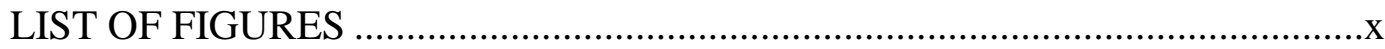

CHAPTER 1: INTRODUCTION, SPECIFIC AIMS, AND STUDY

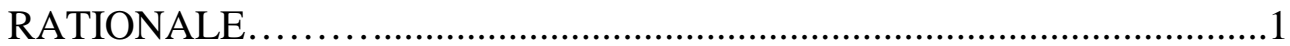

1.1 Introduction and background of the study ........................................ 1

1.2 Background and Context................................................................

1.3 Statement of Purpose .........................................................................

1.4 Specific Aims and Hypotheses .......................................................

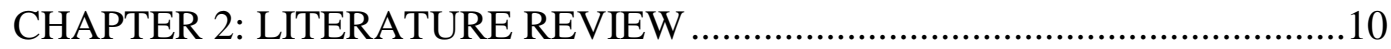

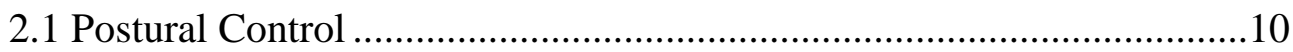

2.2 Suspected Brain Mechanisms Involved in Balance Dysfunction after a

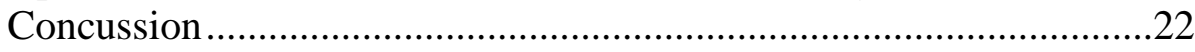

2.3 Return-to-Play Procedure...................................................................31

2.4 Timeline of Balance Recovery after a Concussion Injury ......................33

2.5 Reliability and Validity Evidence of Balance Assessments ..................44

2.6 Reliability and Validity Evidence of the ImPACT Test ........................50

CHAPTER 3: METHODOLOGY AND RESEARCH APPROACH ...................52

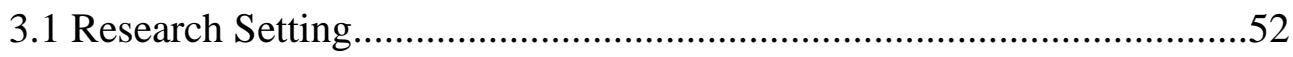

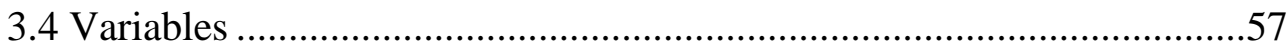

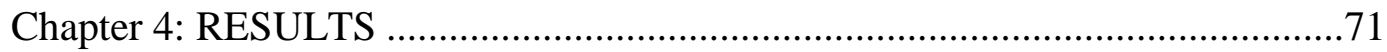

4.1 Static Balance Assessment...............................................................73

4.2 Dynamic Balance Assessment .........................................................76

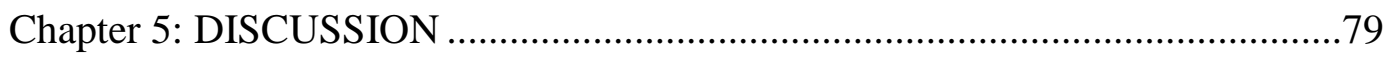

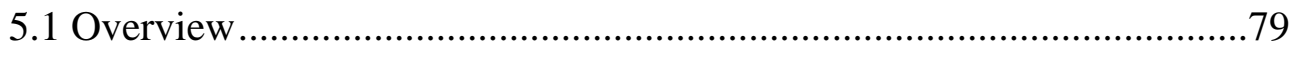

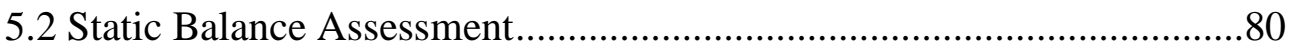

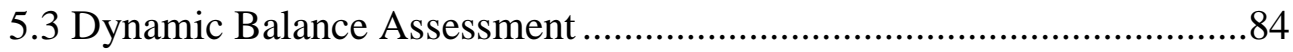

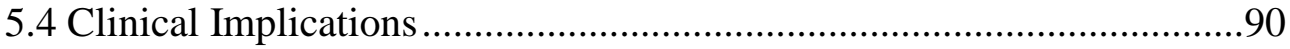


5.5 Study Limitations................................................................................91

5.6 Future Research Considerations ............................................................92

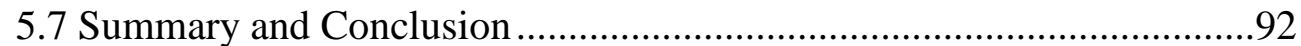

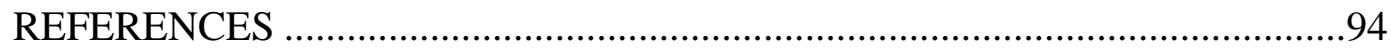

Appendix A: Informed Consent.......................................................................103

Appendix B: Health Status Questionnaire ........................................................108

Appendix C: History Form ………………………........................................112

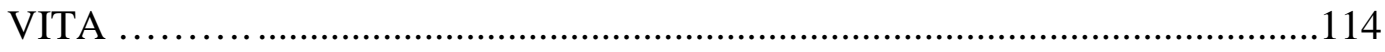




\section{LIST OF TABLES}

Table 1: Inclusion and Exclusion Criteria of Healthy Control Participants .............................. 53

Table 2: Inclusion and Exclusion Criteria for Athletes with Concussions ................................. 54

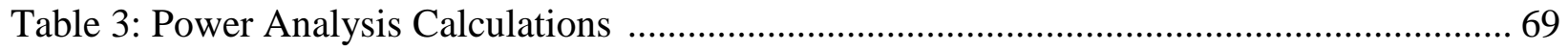

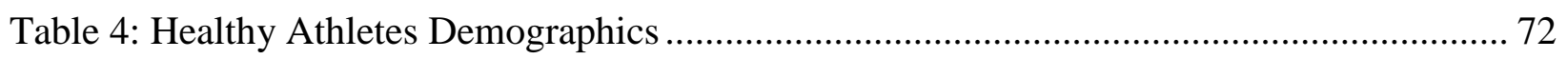

Table 5: Athletes with Concussions Demographics ............................................................. 72 


\section{LIST OF FIGURES}

Figure 1: The Direct Pathway of the Basal Ganglia. ............................................................... 23

Figure 2: The Indirect Pathway of the Basal Ganglia........................................................ 23

Figure 3: The Five Parallel Cerebral Brain Circuits. ............................................................. 25

Figure 4: Graduated Return-to-Play Protocol ....................................................................... 32

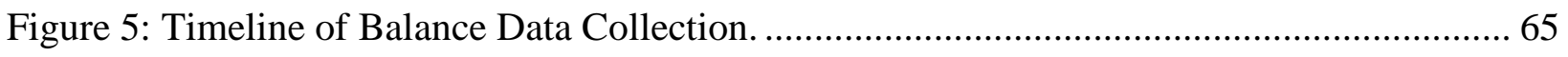

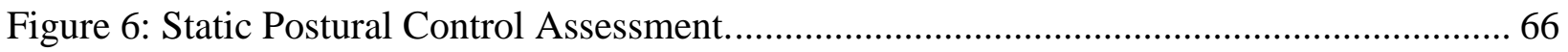

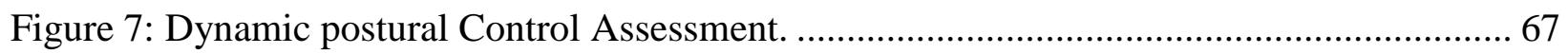

Figure 8: Peak CoP Velocity Means and Standard Deviations in the Eyes Open A/P Direction

During the Quiet Standing Balance Tasks..................................................................... 74

Figure 9: Peak CoP Velocity Means and Standard Deviations in the Eyes Closed A/P Direction

During the Quiet Standing Balance Tasks...................................................................... 75

Figure 10: Peak CoP Velocity Means and Standard Deviations in the Eyes Open M/L Direction

During the Quiet Standing Balance Tasks. .......................................................................... 75

Figure 11: Peak CoP Velocity Means and Standard Deviations in the Eyes Closed M/L Direction

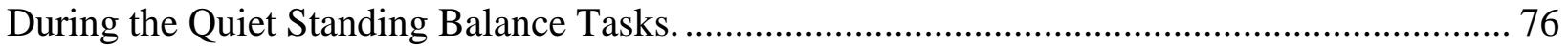

Figure 12: Peak CoP Velocity Means and Standard Deviations in the A/P Direction During the

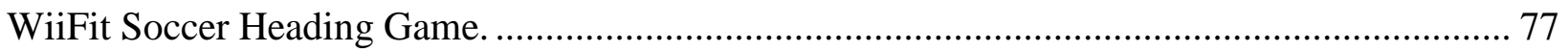

Figure 13: Peak CoP Velocity Means and Standard Deviations in the M/L Direction During the

WiiFit Soccer Heading Game. ................................................................................... 78 


\section{CHAPTER 1: INTRODUCTION, SPECIFIC AIMS, AND STUDY RATIONALE}

\subsection{INTRODUCTION AND BACKGROUND OF THE STUDY}

This study aims to explore two discrete methods of evaluating balance recovery and the underlying motor control mechanisms responsible for postural control (balance) recovery in athletes following a concussion. This research is of particular interest to health professionals given that the neurological mechanisms underlying diminished postural control and timeline of postural control recovery following a concussive closed head injury remain unknown. This research employed a common biomechanical assessment technique and a novel motor control balance assessment method. This biomechanical assessment method is commonly used to evaluate postural control and provide insight into potential mechanisms responsible for progressive recovery of postural control and balance in response to a sport-related concussion.

This chapter will begin with an overview of the epidemiology and background information on sports related concussion injuries, along with current postural control methods used in concussion assessment and research. In addition, the problem statement with a purpose statement, followed by a specific aims and hypotheses section will be presented.

\subsection{BACKGROUND AND CONTEXT}

\subsubsection{Epidemiology and Management of Concussion in Sports}

An estimated 1.6-3.8 million concussion injuries occur in the United States annually with more than 300,000 of those being sport-related (Center for Disease Control and Prevention;

Gessel, Fields, Collins, Dick, \& Comstock, 2007; Marar, McIlvain, Fields, \& Comstock, 2012). One third of all injuries in high school and collegiate athletics are classified concussions, with 
16\% being a secondary concussion (Marar et al., 2012; Ryan, \& Warden, 2003; Slobounov, Slobounov, Sebastianelli, Cao, \& Newell, 2007). Initial or secondary concussions could lead to Second Impact Syndrome, or delayed cerebral edema (Cantu, 1998; McCrory, Davis, \& Makdissi, 2012; Saunders \& Harbaugh, 1984; Weinstein, Turner, Kuzma, \& Feuer, 2013). Second Impact Syndrome is hypothesized as a catastrophic neurological outcome following a concussion injury (Cantu, 1998). The vulnerability exposes the athlete to severe neurological, cognitive, and physiological effects that could translate into lifelong impairments (Cantu, 1998). These impairments severely limit an athlete's overall health and wellness, and in turn reduce their ability to return to play As such, it is critical to ensure the athlete has recovered from a concussion before releasing to play in order to mitigate the chances of having an additional head injury that could lead to Second Impact Syndrome. Further, if an athlete is released too soon, it could increase the probability of the avoidable severe neurological consequences.

Unfortunately, a significant number of sport-related concussions are unreported (Harmon et al., 2013; McCrea, Hammeke, Olsen, Leo, \& Guskiewicz, 2004). Concussion assessment is restricted to athletes who present the signs and symptoms of a concussion and are referred for further assessment (Mulligan, Boland, \& Payette, 2012). The rates of concussions reported by the Center for Disease Control and Prevention (CDC) only include athletes who were admitted to the emergency room and classified as a concussion (Centers for Disease Control and Prevention (CDC), 2006). As such, many players may be participating in sporting activities with an unreported concussion. These undocumented concussions could place athletes at an increased risk of brain hemorrhage or death if they experience a subsequent concussion before the previous concussion has resolved (Gessel et al., 2007). Given that the rate of sport involvement is anticipated to increase at the high school and collegiate levels, a strong need exists for the 
reduction of the prevalence of sport-related concussion (Gessel et al., 2007). An essential component of concussion management is to reduce the number of secondary concussions through the development of evidence-based Return-to-Play (RTP) policies.

The management of a sport-related concussion requires a team of experts from multiple fields of medicine and rehabilitation sciences to accurately diagnose a concussion injury (McCrory et al., 2013). However, the availability of an interdisciplinary team is rare. Most athletic trainers are able to make moderately accurate decisions pertaining to the presence or absence of a concussion injury in an athlete. If properly diagnosed, the athletic trainers then remove the athlete from training or competition. However, the availability of athletic trainers at middle and high school level athletics in the United States is limited (McCrory et al., 2013). As such, the rates of unreported concussion have been reported to exceed $50 \%$ in athletic programs below the collegiate level (McCrea et al., 2004). These high rates can be attributed to limited availability of concussion education programs for parents and coaches of youth sport participants (Garrick, 2005; McCrea et al., 2004). A second potential mechanism for high rates of unreported concussions in athletics below the collegiate level is the absence of an objective assessment tool for sideline use by clinicians (Garrick, 2005; McCrea et al., 2004). Recent media attention to concussion injuries at the professional sporting level and educational programs set forth by the National Football League (NFL) and Federation Internationale de Football Association (FIFA) have expanded global awareness of the signs and symptoms of a concussion (McCrory et al., 2013). However, the need for reliable, cost-effective methods of concussion assessment and reporting is warranted. Furthermore, these assessments should be based upon valid and reliable measurements rather than the traditional clinical judgment. 


\subsubsection{Definition of a Concussion}

Concussion is defined as a complex pathophysiological process affecting the brain, induced by traumatic biomechanical forces (McCrory et al., 2013). Concussion symptoms vary and can include confusion, amnesia, loss of consciousness, loss of spatial and temporal awareness, headaches, migraines, speech impairment, dizziness, nausea, balance disturbances, oculomotor control reduction, impaired vision, gait unsteadiness, and poor coordination (Aubry et al., 2002; McCrory et al., 2005; McCrory et al., 2013). Due to the variety of the symptoms experienced and the multiple mechanisms of injury that can contribute to a concussive injury, no two concussions are the same (Aubry et al., 2002; McCrory et al., 2005; McCrory et al., 2013). This has created a challenge in the assessment and management of sport related concussion.

Presently, no single tool can fully quantify a concussion injury (McCrory et al., 2013). However, measures that focus on the most prevalent symptoms experienced by individuals after a concussion injury could aid in accurate diagnosis and RTP decision making. Dizziness and balance dysfunction have been reported to be some of the most common and debilitating symptoms of a concussion (Guskiewicz, 2011; McCrory et al., 2013). Of individuals suffering from sport-related concussion, 30\% experience balance dysfunction and 75.6\% report dizziness as a debilitating symptom (Guskiewicz, 2011; Marar et al., 2012). Balance dysfunction is the inability to stand with an upright posture without deviating outside the limits of stability or base of support (Guskiewicz, 2011; Horak, 1987). Balance dysfunctions are of particular concern as they may place the athlete at greater risk of falling and/or collisions during game play, leading to additional injury. Balance dysfunctions and dizziness are neurologically related and have been directly linked to physical damage to either the vestibular system or the central cortical areas of the brain (Mucha, Collins, \& French, 2012). Thus, examination of balance dysfunction after a 
concussion injury could provide a quantitative and objective measure of concussion injury and recovery in a large number of individuals with concussions.

\subsubsection{Balance Assessments and Concussion}

Over $75 \%$ of sport-related concussions present with reduced balance or dizziness as determined by current sideline and RTP protocol (Gessel et al., 2007; Marar et al., 2012). A limitation of the current sideline assessments and RTP protocol are the reliance on static and subjective balance measurements. These static assessments, such as the Romberg Test (RT) and the Balance Error Scoring System (BESS), emulate traditional quiet stance measures and lack an unpredictable environmental setting. These measures do not introduce external perturbations and lack the environmental relevance that is found during athletic performance. Accurate evaluations of balance (postural control) should emulate game-like parameters and use environmentally relevant dynamic balance assessments. Dynamic balance assessments seek to replicate an athletic sport match by introducing an unexpected ever-changing environment. Recent literature suggests that athletes who follow the current RTP protocol could be returning to play prematurely with unresolved balance dysfunction (Powers, Kalmar, \& Cinelli, 2013). In addition, the literature reporting on the requisite timeline of balance recovery post-concussion is conflicted. The inconsistencies of the timeline of balance recovery could be due to the lack of sensitive dynamic measures of balance (Bell, Guskiewicz, Clark, \& Padua, 2011; Cavanaugh, Guskiewicz, \& Stergiou, 2005; Guskiewicz, 2011; Powers et al., 2013; Slobounov et al., 2007). The use of novel procedures such as an environmentally relevant dynamic balance task could help refine the timeline for recovery of postural control and aid in mitigating secondary sportrelated concussions. 
The current assessments of balance have come under scrutiny recently by researchers following the NCAA's adoption of the non-traditional methods of examining balance, such as the WiiFit (NCAA sports medicine handbook.2009-2010; Guskiewicz, 2011). Current clinical assessments of balance supported by research are the RT and BESS (Bell et al., 2011; Guskiewicz, 2011). Both the RT and the BESS are low technology assessments of balance that rely upon subjective rater interpretations to evaluate balance and emphasize the maintenance of static balance (Bell et al., 2011; Guskiewicz, 2011; Khasnis \& Gokula, 2003). In both the RT and the BESS, individuals suspected of having a concussion attempt to stand in an upright position and not sway throughout the different conditions. If a sway is detected by the investigator, it counts as a positive RT sign and a higher score on the BESS. However, only the BESS balance test is considered reliable and valid for use in sport-related concussion injuries (Bell et al., 2011). Furthermore, the BESS balance test is the current balance assessment used in making RTP decisions (Bell et al., 2011; McCrory et al., 2013). Balance, measured by the RT and the BESS, post-concussion is postulated to return to normal baseline measurements within 72 hours (Bell et al., 2011; Guskiewicz, 2011). However, the use of laboratory grade force platforms along with environmentally relevant dynamic balance tasks has determined that residual balance dysfunction exists 1-4 weeks post-injury (Cavanaugh et al., 2005; Powers et al., 2013; Slobounov et al., 2007). No known literature to date has examined laboratory grade force platforms dynamic balance assessments and the traditional clinical static balance assessments in the same sample of athletes with concussions. Until this is accomplished the timeline of balance recovery reported by the literature is inconsistent and needs to be evaluated further using quantitative, reliable, and novel techniques. 
The WiiFit Soccer Heading Game is a low cost game system that uses lower body ground reaction force (GRF) and center of pressure (CoP) data to complete specific goals based upon the game’s requirements. In addition, it uses on screen visual stimuli with representative avatars and icons to direct one's center of pressure to meet dynamic changing environmental conditions. The central nervous system‘s (CNS) ability to regulate and weight incoming sensory information in response to rapidly changing tasks and environmental conditions is necessary for proper postural responses. Proper postural responses are exhibited through efficient and correct interaction of the three major sensory systems; the eyes, the vestibular system, and the somatosensory system. In the maintenance of dynamic postural control, the system relies upon all three sensory systems to properly control upright posture in the presence of expected and unexpected environmental conditions. For an athlete returning to competitive play, evaluation of postural control should include the dynamic unanticipated environmental condition that the athlete is expected to return to. Traditional balance assessments that seek to emulate quiet stance fall short in evaluating postural control in these environmental conditions. Instead, the WiiFit Soccer Heading Game may provide a simple and cost effective tool that closely resembles the functional demands of an athletic contest that stresses the three major sensory systems responsible for postural control.

\subsection{Statement OF PURPose}

Therefore, the purpose of this study was to investigate changes in postural control during a traditional quiet stance balance assessment and an environmentally relevant dynamic balance assessment twice during one week of recovery in the same sample of athletes with concussions. Furthermore, track performance on these two types of assessments post-concussion to determine their relationship and sensitivity to the recovery of a concussion. This research sought to quantify 
environmentally relevant dynamic balance tasks during recovery from a sport-related concussion. The aim of this research was to determine if a dynamic experimental procedure can assess balance impairment in athletes with concussions when compared to a common method of static balance assessment in athletes with concussions.

\subsection{SPECIFIC AIMS AND HYPOTHESES}

The specific aims of this study were:

Aim 1: To compare recovery of postural control during a traditional quiet stance balance assessment and an environmentally relevant dynamic balance assessment, the WiiFit Soccer Heading Game, twice during one week recovery period in athletes with concussions.

Hypothesis 1: We hypothesized that athletes with concussions recover static balance within one week post-injury whereas the dynamic balance assessment balance impairment would persist beyond one week.

Aim 2: To quantify the difference between healthy individuals and athletes with concussions postural control during the administration of a quiet stance balance assessment and an environmentally relevant balance task.

Hypothesis 2A: We hypothesized that if an environmentally relevant balance assessment, WiiFit Soccer Heading Game, is a more challenging motor control task for athletes with concussions when compared to healthy athletes. Then greater CoP Peak Velocity will be observed in both the mediolateral (M/L) and anteroposterior (A/P) directions, when compared to the static quiet stance balance assessment, in athletes with concussions. 
Hypothesis 2B: We hypothesized that the static balance assessment will be a simple motor control task, as measured by increased CoP velocity in the A/P direction only in athletes with concussions when compared to the environmentally relevant balance assessment. 


\section{CHAPTER 2: LITERATURE REVIEW}

The following is a review and discussion of the theory of postural control and its relationship to a concussion injury. In addition, potential brain mechanisms responsible for balance dysfunction, current RTP protocol, and the current timeline for the recovery of balance dysfunction is explored. Furthermore, reliability and validity evidence for traditional measures of quiet stance, the BESS and RT, along with the WiiFit, and the Immediate Post-Concussion Assessment and Cognitive Test (ImPACT Test) in individuals with concussions is reviewed.

\subsection{Postural Control}

Postural control in humans has been defined as the ability to maintain equilibrium and orientation in a gravitational environment (Horak, 1987). In an erect posture, two legged stance, humans are in a constant state of unstable equilibrium and must counter the effects of gravity continually through tonic muscular control (Horak, 1987; Shumway-Cook \& Horak, 1986; Shumway-Cook \& Horak, 2012). Postural control is a complicated process that involves an individual, a task, and the effect of the environment on the task (Horak, 1987; Shumway-Cook \& Horak, 2012). In order to maintain postural control, the visual, vestibular, and somatosensory systems actively make corrections of posture through reflexive and direct muscular contractions.

In an erect posture, postural stability or balance is controlled by the maintenance of the center of mass (COM) in relation to the base of support (BOS) (Guskiewicz, 2011; Horak, 1987;

Riley, Mann, \& Hodge, 1990; Shumway-Cook \& Horak, 1986; Shumway-Cook \& Horak, 2012). Center of mass is the central point of the total body mass, and its vertical projection is also known as the center of gravity (COG) (Shumway-Cook \& Horak, 2012). The BOS is defined as the area of the body that is in contact with the ground in standing (Horak, 1987; Shumway-Cook \& Horak, 2012). The theoretical projection of COM lies within the BOS during upright quiet 
standing (Horak, 1987; Riley et al., 1990). The control of the COM relative to the BOS is of great interest for researchers in motor control, posture, and balance as it provides insight into the motor control processes behind postural control (Horak, 1987; Riley et al., 1990; ShumwayCook \& Horak, 1986). More importantly, the relationship between COM and BOS has been used as a reliable and valid quantitative assessment in concussion research (Cavanaugh, Mercer, \& Stergiou, 2007; Guskiewicz, 2011; Slobounov et al., 2007).

\subsubsection{Models of Postural Control}

Multiple models were created to help researchers explain the control of the COM in relationship to the BOS during erect posture. The models of postural control further explain how the body accounts for its position in relationship to its internal environment and the external environment. Due to the ever changing external environment, the human body is in a constant state of adjusting and adapting with respect to the capacity and position of the structures of the body. The body must account for its position with respect to the environment in order to prevent an unexpected fall (Shumway-Cook \& Horak, 1986). The models range from simplistic integrations of feed-back control, also known as cause and effect control, of the sensory systems to more complicated feed-forward (anticipatory control) systems or a combination of both (Klous, Mikulic, \& Latash, 2011; Riley et al., 1990; Winter, 1995). Three major models have been used to explain the feed-backward and feed-forward mechanisms of postural control. They are the inverted pendulum model, sensorimotor model, and anticipatory postural adjustments model (Klous et al., 2011; Riley et al., 1990; Winter, 1995). 


\subsubsection{Inverted Pendulum Model}

The inverted pendulum model of postural control is the basis for understanding upright bipedal or two legged stance. The body's responses to small disturbances of stance posture and the ability to evenly distribute one's weight in an upright and steady position is the fundamental principle of the inverted pendulum model (Riley et al., 1990). An example of an inverted pendulum model can be illustrated by imagining a bowling ball (the head and upper body) placed directly on top of a chopstick (lower body). Minor fluctuations in either anterior or posterior position of the bowling ball will require effort on the part of the chopstick to maintain its equilibrium. Our human body is essentially this inverted pendulum since two-thirds of our body mass is located at two-thirds our body height (Winter, 1995). If our body sways anteriorly our COM moves outside of the BOS and forces a muscular postural response in order to prevent a fall. This response is observed as an increased activation of the plantar flexor muscles (e.g. the gastrocnemius, soleus, plantaris, flexor hallucis longus, flexor digitorum longus, and the tibilalis posterior). Increased muscle activation projects the CoP beyond the $\mathrm{COM}$ in order to move it back into the BOS (Horak, 1987; Winter, 1995). Winter (1995) observed this phenomenon while a subject swayed back and forth at a regular interval while standing on a force platform. As the COM moves during quiet stance, the CoP continuously moves around the COM in an attempt to keep the COM within the BOS (Guskiewicz, 2011; Horak, 1987; Shumway-Cook \& Horak, 2012). The muscular contractions by the plantar flexor muscles work synergistically in both the $\mathrm{A} / \mathrm{P}$ and $\mathrm{M} / \mathrm{L}$ direction to move the $\mathrm{CoP}$ in response to the COM. In a simple analogy, CoP is like a sheep dog that attempts to herd a group of sheep (COM) in a given area (BOS). This analogy provides the picture that as the body sways and attempts to maintain equilibrium, the CoP chases and maintains the COM within the BOS in order to prevent a fall (Winter, 1995). 
The inverted pendulum model is a simple approximation of postural control. It aids in the basic understanding of postural control because it portrays the muscles’ role in moderating balance. However, the model fails to incorporate how the CNS approximates the shift of the COG and moves the CoP to protect against a fall. Sensory systems that are regulated by the CNS, through direct muscle control, need to be incorporated when modeling the maintenance of postural control.

\subsubsection{Sensorimotor Model}

The sensorimotor model elaborates on the inverted pendulum model to incorporate the sensory systems contributions to postural control. Upright bipedal stance is achieved by the visual, vestibular, and somatosensory systems detection of sway and the provision of feed-back signals (Peterka, 2002). This provision produces the appropriate amount of muscular torque to adjust for the body motion sway in order to prevent a fall (Peterka, 2002). However, in certain situations, sensory information is inhibited or reduced due to environmental stimuli or sensory system degradation resulting from the natural aging process.

The sensorimotor model has been observed and explained in individuals with vestibular loss who have experienced an acoustic neuroma or age-related neuropathy. Because of the loss of vestibular feed-back, these individuals demonstrated an increase in muscular stiffness and a reduction of CoP excursion during quiet stance when compared to healthy individuals (Peterka, 2002). In the absence of vestibular feed-back cues to aid in the determining the bodies position, in the environment, the visual and somatosensory systems are solely relied upon to interpret environmental information. This reliance makes the postural control system less efficient and more prone to an unexpected fall. Integration of the visual, vestibular, and somatosensory 
sensory systems information drives the feed-back signals needed for proper postural control. As environmental conditions become more chaotic, such as during a sporting event, driving a car or walking, increased stress is applied to the sensory systems. The sensorimotor loading and integration between the systems are critical in determining body positioning in space in order to apply appropriate muscular responses (Peterka, 2002). Deficits in the integration of the sensory systems could increase the displacement of the COM outside of the BOS and induce a fall (Peterka, 2002).

The sensorimotor model expands upon the inverted pendulum model because it takes into account how the sensory systems, in conjunction with the actual sensation, aids in controlling erect posture. Furthermore, the sensorimotor model explains the higher brain centers ability to regulate postural control through direct interaction with the sensory systems. This explains how the CNS regulates postural control through the generation of muscular responses in the presence of environmental stimuli. A limitation of this model is that it fails to account for the body's ability to anticipate and prepare for an environmental stimulus. The CNS system can regulate postural control by direct feed-back and feed-forward (anticipatory) signals from the sensory systems based upon prior the learning of past experiences.

\subsubsection{Anticipatory Postural Adjustments Model}

Anticipatory postural adjustments (APA) model properly explains the feed-forward mechanism of postural control. In the presence of an expected perturbation, the body anticipates the event (Klous et al., 2011). The anticipation is characterized by voluntary movements of the body segments such as increased postural muscles activation prior to an event (Klous et al., 2011). For example, when an upright individual quickly raises one arm voluntarily, postural 
muscles activate, such as the gastrocnemius and tibialis anterior, approximately 100 ms prior to the movement of the arm (Klous et al., 2011; Massion, 1992). The anticipatory postural adjustment provides the necessary preparation of the body for an event in order to prevent a fall. Feed-forward control systematically alters postural control in order to meet the demands placed upon the body in accordance to the environment.

A neural controller is hypothesized to command and regulate the magnitude of the anticipatory reactions in a given context (Klous et al., 2011). This suggests that instead of a bottom up or a reflexive feed-back control of erect posture as observed in the inverted pendulum and sensorimotor model, top down organization is also present. This higher center neural organization is a learned process that requires extensive sensory information and prior knowledge of set situations. The APA modifies the magnitude of the signaling response given this foreknown knowledge. For example, if an athlete is throwing a ball to a moving target a postural muscle response in the lower limbs and the core is expected in order to brace and anticipate the release of the ball from the arm. This anticipated muscular response prevents an unexpected fall due to the sudden change in the position of the COM due to motion of the arm.

The combination of feed-forward and feed-backward signals are necessary for preparing the appropriate muscular responses of the body for unexpected and expected environmental conditions. Feed-forward control anticipates a given event, while feed-backward control modifies and adjusts the control system as the event occurs. As the system becomes more refined and learned, simple environmental cues create APA responses. These responses's aid in the reduction of fall risks in the presence of an unexpected perturbation.

The combination of an inverted pendulum, sensorimotor, and the anticipatory postural adjustments models explain the body's ability to regulate postural muscle responses 
during a given task in a certain environment. The ability to regulate these muscular responses relies heavily on the visual, vestibular, and somatosensory systems cues. Proper integration and weighting of the sensory systems should provide the CNS with the needed information to execute the proper postural control response. In individuals who have sensory system dysfunction or degradation from a traumatic neurological event, a reduction in the feed-back and feed-forward mechanisms is expected (Klous et al., 2011). This interruption will influence how accurately the body responds to environmental stimuli. Thus, a proper examination of postural control is performed by stressing the feed-back and feed-forward capabilities of the CNS. Only through this stress in a functional setting, such as the replication of game-like scenarios, will one’s postural control system dysfunction be exposed and documented.

\subsubsection{Calculations of Postural Control}

Comprehension of postural control models should focus on not only the model, but the forces deriving the model. The maintenance of COM by the CNS is through the generation of muscular forces of the postural muscles (Horak, 1987; Shumway-Cook \& Horak, 1986; Shumway-Cook \& Horak, 2012). The generation of muscular forces can be directly measured through multiple parameters, yet the most common way of an assessment is through center of pressure (CoP). Center of pressure is the center of distribution of the total forces applied to a supporting surface (Shumway-Cook \& Horak, 2012). As the COM moves during quiet stance, the CoP continuously moves around the COM in an attempt to keep the COM within the BOS (Guskiewicz, 2011; Horak, 1987; Shumway-Cook \& Horak, 2012). In a simple analogy, CoP is like a sheep dog that attempts to herd a group of sheep (COM) in a given area (BOS). This analogy provides the picture that as the body sways and attempts to maintain equilibrium, the CoP chases and maintains the COM within the BOS in order to prevent a fall. 
Center of Pressure calculations are based upon a central idea that motor control processes are hierarchical and contain two levels or orders (Masani, Popovic, Nakazawa, Kouzaki, \& Nozaki, 2003; Prieto, Myklebust, Hoffmann, Lovett, \& Myklebust, 1996; Zatsiorsky \& Duarte, 2000). The highest order determines the reference location of the system (the body) with respect to how equilibrium is maintained (Zatsiorsky \& Duarte, 2000). Lower order is the muscular maintainer of balance (Zatsiorsky \& Duarte, 2000). In other terms, higher order calculations of $\mathrm{CoP}$ are postulated to approximate the neural control mechanisms that are involved in the maintenance of postural control. While lower order $\mathrm{CoP}$ calculations measure the muscular responses, or the direct controller, of postural control.

The computation of CoP is complicated but is derived from the ground reaction force (GRF) during bipedal stance. The GRF is the force exerted by the ground pushing against the foot. This force vector is three-dimensional and consists of a vertical component $\left(\mathrm{F}_{\mathrm{Z}}\right)$ and two shear components $\left(\mathrm{F}_{\mathrm{X}}, \mathrm{F}_{\mathrm{Y}}\right)$ (Winter, 1995). The shear components are typically referred to as anterior-posterior $\left(\mathrm{F}_{\mathrm{X}}\right)$ and medial-lateral $\left(\mathrm{F}_{\mathrm{Y}}\right)$ directions (Winter, 1995). In addition to the force vectors, three-dimensional force moments (the force acting about a point or axis) are given by the force platform in the anterior-posterior $\left(\mathrm{M}_{\mathrm{X}}\right)$, medial-lateral $\left(\mathrm{M}_{\mathrm{Y}}\right)$, and vertical $\left(\mathrm{M}_{\mathrm{Z}}\right)$ directions (Winter, 1995). The common calculation of CoP in the anterior-posterior (AP) direction is a time varying calculation based upon the CoP coordinates returned from the force platform. Center of Pressure in the anterior-posterior direction is calculated using the following equation:

$C o P_{A P}=\frac{-M_{Y}+\left(F_{X^{*}} d z\right)}{F_{Z}}$, where $\mathrm{dz}$ pertains to the distance from the surface to the force platform origin (Klous et al., 2011). The CoP in the anterior-posterior direction refers to the amount of movement or sway in the anterior-posterior direction. The calculation of CoP in the medial- 
lateral (ML) direction is time varying and is calculated by the same equation, albeit the substitution of the $\mathrm{F}_{\mathrm{X}}$ and $\mathrm{M}_{\mathrm{Y}}$ is necessary. The equation for the calculation of $\mathrm{CoP}$ in the $\mathrm{ML}$ direction is as follows:

$C_{o} P_{M L}=\frac{-M_{X}+\left(F_{Y} * d z\right)}{F_{Z}}$, where $\mathrm{dz}$ pertains to the distance from the surface to the force platform origin (Klous et al., 2011).

Multiple calculations exist for processing the CoP coordinates. However, three main post-processing computations of CoP coordinates are commonly reported for the analyses of postural control in concussion; displacement, velocity, acceleration (Cavanaugh et al., 2005; Guskiewicz, 2011; Powers et al., 2013; Slobounov, Tutwiler, Sebastianelli, \& Slobounov, 2006). Displacement is a time series dependent calculation $\left(d=v_{i} * t+\frac{1}{2} * a * t^{2}\right.$ or $\left.d=\frac{v_{i}+v_{f}}{2} * t\right)$ that approximates the entire distance the CoP traveled during a given time period. The displacement of CoP in either the AP or ML direction is usually computed by subtracting the average CoP (AP or ML) coordinate during quiet stance from the CoP (AP or ML) coordinate in the other desired trials (Klous et al., 2011). Displacement is a lower order CoP calculation because it represents the muscular forces that are responsible for controlling the position of human body (Menegoni et al., 2011; Powers et al., 2013). Whereas, Velocity $(v=\Delta d / \Delta t)$ and acceleration $(\mathrm{a}=\Delta \mathrm{v} / \Delta \mathrm{t})$ are a higher order CoP calculations that estimate the variables coding direction and intensity of movement (Nashner, Black, \& Wall, 1982; Powers et al., 2013; Winter, 1995). Velocity and acceleration are higher frequency movements that require greater sensory integration from the visual and vestibular systems, whereas displacement is controlled principally by the somatosensory system (Masani et al., 2003; Prieto et al., 1996). As such, sway velocity has been denoted as a more sensitive measure when assessing postural control, specifically in neurologically impaired individuals (Guskiewicz, 2011). In addition, CoP 
acceleration is typically used to determine the onset of sway because it is more susceptible to changes in velocity over time (Winter, 1995). Center of Pressure acceleration is not as widely used as velocity in concussion research. This is because it is of interest for researchers to quantify the amount of sway, rather than the point at which sway occurs. In addition, no pathology currently exists to link the dysfunction in the onset of sway (acceleration), but the amount of sway (displacement) and the control of sway (velocity) have been observed in athletes with concussions.

The final computed CoP in both the AP and/or medial-lateral (ML) CoP directions can be summated and graphed. This summated CoP produces a two dimensional "spaghetti" diagram of CoP that demonstrates the control of ones COM (Horak, 1987; Winter, Patla, Ishac, \& Gage, 2003). This spaghetti plot can be either measured in CoP AP or ML displacement or velocity. The distribution of CoP spaghetti plots provides information as to how the COM is controlled during a movement (Horak, 1987; Winter, 1995). The greater the displacement of the plot, along with higher CoP velocity, could indicate decreased steadiness during quiet stance (Ruhe, Fejer, \& Walker, 2011). Conversely, the tighter the plot and the lower the CoP sway velocity could indicate greater steadiness during quiet stance (Ruhe et al., 2011). However, it should be noted that underlying movement patterns in the AP and ML directions separately on the spaghetti plot could provide additional information that is not expressed in the summated CoP spaghetti plots. Thus, examination of the summated CoP spaghetti plot and the dichotomized AP and ML CoP spaghetti plots is warranted. 


\subsubsection{Model of Postural Control Recovery in Concussion}

The goal of the commonly used clinical balance assessments in concussion research is to remain in a quiet standing position (Guskiewicz, 2011). For example, the RT and the BESS require that the person remain as still as possible in an upright position during evaluation in an unmoving environment. Similar to static CoP, these tests attempt to describe postural control based upon little to no external stimuli (Cavanaugh et al., 2005). The lack of environmental stimuli cannot predict postural control mechanisms adaptations in multi-sensory unpredicted environments (Cavanaugh et al., 2005). Simplistic measurements and models of postural control do not adequately describe the functional or dynamic based balance problems exhibited after a concussion injury.

Current lack of proof to show that individual sensory systems are impaired after a concussion is well noted, but not well accepted (Guskiewicz, 2011). Furthermore, recent evidence demonstrates that disruption in the interconnectedness of all the sensory systems points to sensory integration errors of the cortical brain after a concussion injury (Cavanaugh et al., 2005; Lovell, 2009; Murray, Ambati, Salvatore, \& Reed-Jones, 2014; Slobounov et al., 2007; Slobounov, Gay, Johnson, \& Zhang, 2012). The sensory integration and interaction errors are only observed in postural control maintenance during functional environmentally relevant dynamic assessments. Recent research postulates that damage from a cerebral concussion is functional rather than structural (Lovell, 2009; Shaw, 2002). This functional disruption is observed in the interruptions of neurometabolic functions, known as the metabolic cascade, such as abrupt neuronal depolarization and changes in glucose metabolism (Blennow, Hardy, \& Zetterberg, 2012). These changes can be directly measured via the leak of metabolic proteins into the peripheral blood circulation that are known as biomarkers. These disruptions in 
neurometabolic function could adversely affect the brain's ability to further excite or inhibit certain structures of the brain involved in the maintenance of postural control. More specifically, the feed-back and feed-forward signals to the areas of the brain responsible for postural control could be interrupted. During a challenging task in a rapidly changing environment, the CNS has the ability to weigh incoming sensory information and apply muscular contractions in order to regulate postural control (Guskiewicz, 2011; Klous et al., 2011; Shumway-Cook \& Horak, 1986). Although dynamic situations are physically and cognitively demanding, feed-back and feed-forward mechanisms of postural control are simultaneously stressed. This stress could expose functional deficits that are not present during static postural control. For an athlete returning to competitive play, it is necessary to assess postural control during an environmentally relevant dynamic task.

Quiet stance during an athletic event is not a desired goal. Most athletics involves a series of simultaneous tasks in a dynamic changing environment. As such, models that measure concussion recovery with static quiet stance as the desired goal are inaccurate. This is due to the multiple interconnected processes and interactions of the neuromuscular, musculoskeletal, and cognitive systems during dynamic tasks (Cavanaugh et al., 2005). The goal of an environmentally relevant dynamic model of predictive postural control is to maintain an upright posture during multiple sensory perturbations with specific goals. Thus, a dynamic model of predictive postural control as evaluated during dynamic game-like parameters is essential in the evaluation of balance dysfunction after a suspected concussion injury. Based upon the known reductions of feed-back and feed-forward control after a concussion injury, but without methods to directly measure this reduction, a need exists to indirectly measure higher order sensory integration. Through direct measurement of second order (velocity) CoP coordinate calculations 
and gaze behavior during a dynamic assessment, the underlying motor control pattern of postural control recovery post-concussion could be more accurately examined.

\subsection{SUSPECTED BRAIN MECHANISMS INVOLVED IN BALANCE DYSFUNCTION AFTER A CONCUSSION}

It is unknown what mechanisms of postural control are damaged in athletes with concussions. Two major mechanisms are suspected to be interrupted during a concussion injury: cortical (basal ganglia loops) and the vestibular system (vestibular nuclei or semicircular canals).

\subsubsection{Basal Ganglia Contributions to Concussion Balance Dysfunction}

The basal ganglia are a group of nuclear masses that reside at the base of and deep within the cerebral hemispheres (Alexander \& Crutcher, 1990; Kandel, Schwartz, \& Jessell, 2000; Lezak, Howieson, \& Loring, 2004). The basal ganglia has two major pathways; direct and indirect (Alexander, DeLong, \& Strick, 1986; Alexander \& Crutcher, 1990; Knierim, 2012). The primary function of the direct pathway is excitation of thalamic neurons (Figure 1), and the indirect pathway’s primary function is the inhibition of the thalamic neurons (Figure 2) (Alexander \& Crutcher, 1990). Beyond the pathways, the basal ganglia projections extend to target zones within the ventrolateral thalamus; along with influences of cortical association and sensorimotor regions through segregated re-entrant pathways that link cortex, basal ganglia, and thalamus (Alexander \& Crutcher, 1990). This link provides the evidence to suggest that a number of cortical circuits pass through and can be influenced by the basal ganglia (Knierim, 2012). 


\section{Cerebral Cortex}

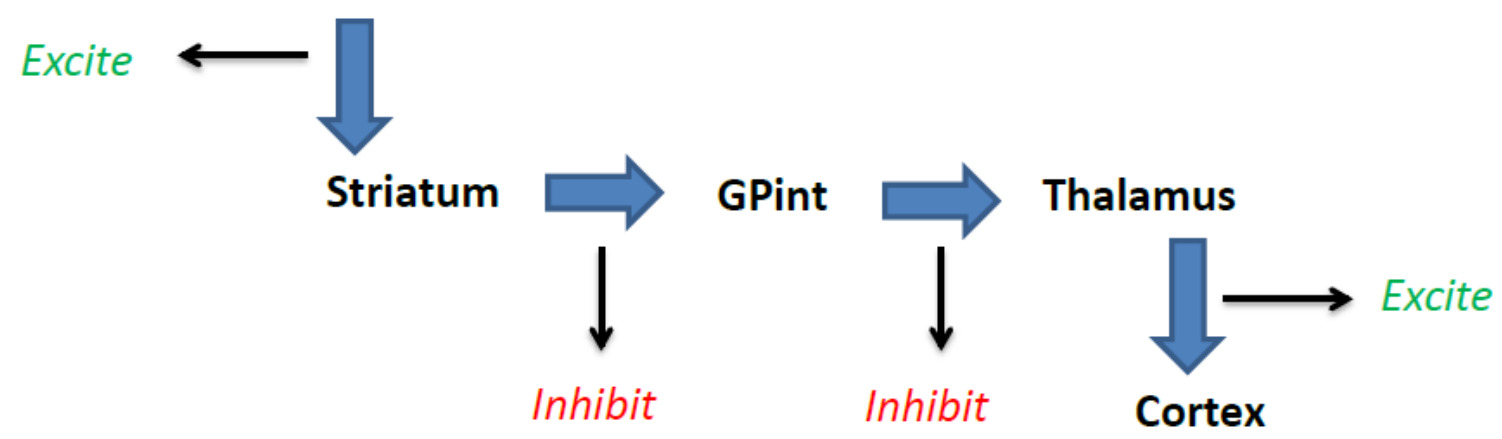

Figure 1: The direct pathway of the basal ganglia.

Notes: Notice that the final result of the pathway is to further excite the motor cortex, thus creating a positive feed-back loop between the basal ganglia and the motor cortex. Note: the large blue arrows are the neurons connecting each structure of the brain in the pathway. Corresponding arrows indicate the function of the neurons such as inhibition or excitation. . Note: GPint = Globus pallidus internal segment . Adapted from Alexander et al., 1990.

\section{Cerebral Cortex}

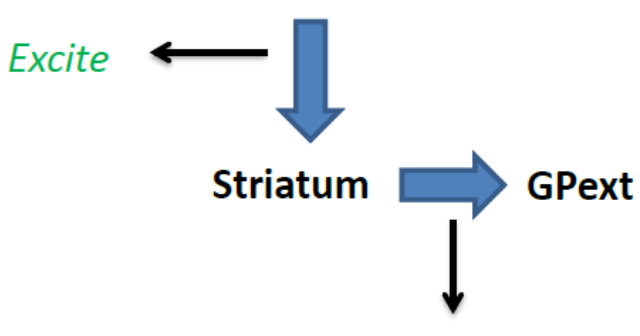

Inhibit

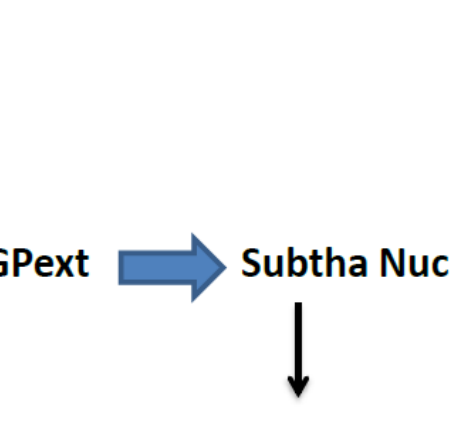

Inhibit
Excite Inhibit

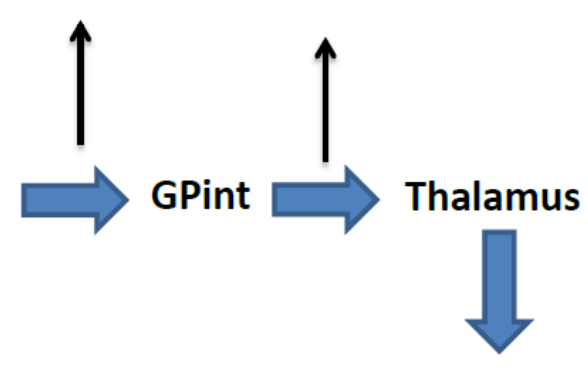

Inhibit

\section{Cortex}

Figure 2: The indirect pathway of the basal ganglia.

Notes: Notice the final result of the pathway is to inhibit the motor cortex thus creating a negative feedback loop between the basal ganglia and the motor cortex. Note: the large blue arrows are the neurons connecting each structure of the brain in the pathway. Corresponding arrows indicate the function of the neurons such as inhibition or excitation. Note: GPext $=$ Globus pallidus external segment, Subtha Nuc = Subthalamic Nucleus, GPint = Globus pallidus internal segment. Adapted from Alexander et al., 1990. 
The five parallel segregated circuits involve motor and non-motor regions of the cerebral cortex (Alexander, DeLong, \& Strick, 1986) (Figure 3). The motor components are the skeletomotor and oculomotor areas of the cortex, while the non-motor areas in the frontal lobe are cognitively related (Alexander \& Crutcher, 1990). The targets of the frontal lobe and the corresponding cortical areas that receive output from the basal ganglia-thalamocortical circuit are limbic, motor, oculomotor, prefrontal, and sensorimotor. The limbic system projections consist of the anterior cingulate area and the medial orbitofrontal cortex (Alexander \& Crutcher, 1990). The motor system projections consist of primary motor area and the supplementary motor area (Alexander \& Crutcher, 1990). The oculomotor system projections consist of supplementary eye field and the frontal eye field (Alexander \& Crutcher, 1990). The prefrontal system projections consist of the dorsolateral prefrontal cortex and the lateral orbitofrontal cortex (Alexander \& Crutcher, 1990). Lastly, the sensorimotor projections consist of the vestibular, somatosensory, and visual (occipital included) sensory segments of the cortex (Alexander \& Crutcher, 1990). Injury of the basal ganglia or the area of the circuits of the cerebral cortex that receive output from the basal ganglia could greatly influence motor and non-motor functions of the body (Alexander \& Crutcher, 1990). 


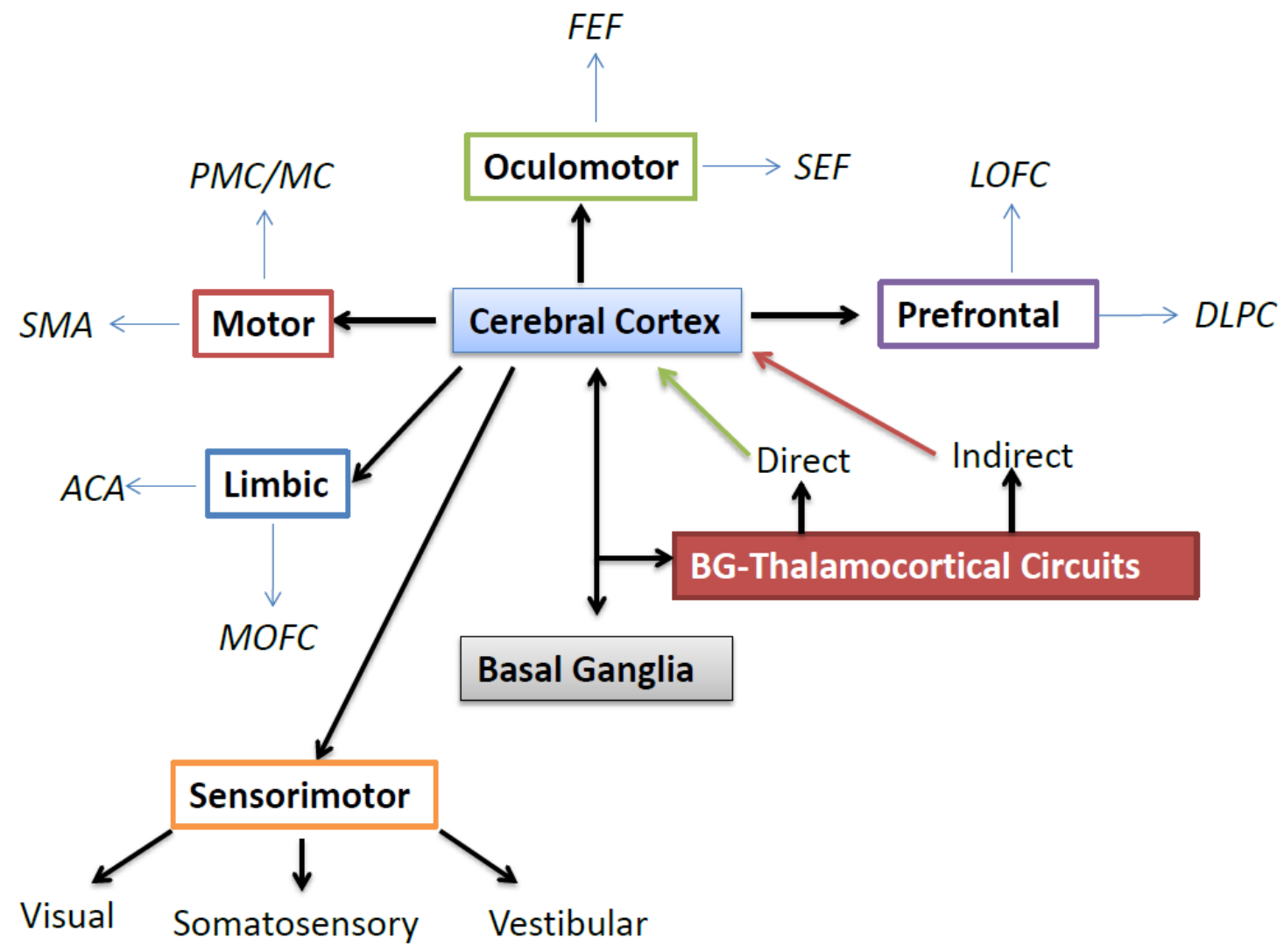

Figure 3: The five parallel cerebral brain circuits that receive direct output from the Basal Ganglia via the Basal Ganglia-Thalamocortical circuits.

Notes: The abbreviations are (starting with the limbic category): $\mathrm{MOFC}=$ medial orbitofrontal cortex; ACA = anterior cingulate area; SMA = supplementary motor area; $\mathrm{PMC} / \mathrm{MC}=$ premotor cortex/motor cortex; FEF = frontal eye field; SEF = supplementary eye field; LOFC = lateral orbitofrontal cortex; DLPC $=$ dorsolateral prefrontal cortex. Note: the green lines indicate excitation and the red line indicates inhibition. Thus, showing the ability of the basal ganglia to either excite or inhibit cerebral cortex neurons. The two way black arrows indicate a feed-back and feed-forward mechanisms of the basal ganglia with the cerebral cortex.

As the brain shifts within the skull, it comes into contact with the skull. This direct contact will produce damage to the brain cells which in turn can cause swelling, axonal shearing, or a neurological lesion (Signoretti, Lazzarino, Tavazzi, \& Vagnozzi, 2011; Slobounov, Gay, Johnson, \& Zhang, 2012). Brain swelling or cerebral edema can be a life threatening condition that is a direct result of cerebral trauma or hemorrhages (Adukaskiene, Bivainyte, \& Radaviciute, 
2007). This swelling will constrict areas of brain tissue and thus result in decreased brain activity in the given area (Adukaskiene, Bivainyte, \& Radaviciute, 2007). Axonal shearing is direct trauma to neurons that result in swelling of the axon and disconnection from the cell body (Alder, Fujioka, Lifshitz, Crockett, \& Thakker-Varia, 2011; Kandel, Schwartz, \& Jessell, 2000). This disconnect will also result in an uncontrolled release of neurotransmitters into the synapse. In high concentrations these neurotransmitters are considered to be toxic to the surrounding neurons (Alder, Fujioka, Lifshitz, Crockett, \& Thakker-Varia, 2011). Thus, direct damage to the neurons via a coup-countercoup injury, such as a concussion, can cause axonal sheering and apoptosis of surrounding neurons (Alder, Fujioka, Lifshitz, Crockett, \& Thakker-Varia, 2011). Lastly, a neurological brain lesion, cell death, could develop after a concussion, due to the impact of the brain with the skull (Signoretti, Lazzarino, Tavazzi, \& Vagnozzi, 2011). All of the above mentioned brain conditions will influence brain functioning in one manner, or another. However, the extent and localization of the damage will result in different observed symptoms. Concussion injuries are variable across and within individuals (McCrory et al., 2013). Thus, damage is not typically seen in the same area of the brain when examining multiple concussion injuries. However, given the extensive reach across the frontal region of the cerebral cortex and throughout the occipital and temporal regions, the basal ganglia loops that influence cortical processing through the parallel cerebral brain circuits could be damaged. Temporary or permanent damage to any of the cerebral brain circuits that receive output from the basal ganglia-thalamocortical circuit will interrupt feed-back control by the basal ganglia. This interruption can influence many different motor and non-motor processes.

Non-motor cortical damage from a concussion injury will result from prefrontal or limbic cerebral basal ganglia loops damage. Damage to the prefrontal loop includes the dorsolateral 
prefrontal cortex (DLPC) or the lateral orbitofrontal cortex (LOFC), and it will influence executive functioning tasks, such as cognition and problem solving (Alexander, DeLong, \& Strick, 1986; Alexander \& Crutcher, 1990). Damage to the limbic brain loop includes anterior cingulate area (ACA) and the medial orbitofrontal cortex (MOFC) (Alexander, DeLong, \& Strick, 1986; Alexander \& Crutcher, 1990). The limbic system is critical in memory, planning, and higher order concept formation (Kandel, Schwartz, \& Jessell, 2000). If damaged, any or all of the aforementioned brain areas will interrupt cognitive brain functioning tasks. Furthermore, it is conjectured that the basal ganglia output will be inhibited due to this damage. Thus, interruption of cortical brain areas will inhibit the excitation of the direct or indirect pathways of the basal ganglia to moderate cognition.

Motor cortex damage from a concussion injury will result in damage to the motor, oculomotor, and sensorimotor cerebral basal ganglia loops. Motor cortex damage includes cell damage to the supplementary motor area (SMA) and/or the premotor cortex/motor cortex (PMC/MC) (Alexander \& Crutcher, 1990; DeLong, Alexander, Mitchell, \& Richardson, 1984). The resultant damage is best observed in pyramidal tract disorders like cerebral palsy (DeLong, Alexander, Mitchell, \& Richardson, 1984). Abnormal motor function such as difficulty with voluntary movements with an increase in spastic involuntary muscle behavior is typical of motor cortex damage. Oculomotor loop damage will include the frontal eye field (FEF) and the supplementary eye field (SEF) (Alexander \& Crutcher, 1990). Abnormal saccades and smooth pursuit will be affected along inputs from the vestibular ocular reflex (VOR) (MacAvoy, Gottlieb, \& Bruce, 1991). Thus, inaccurate motion detection and balance impairment are symptoms that could occur from this damage. Lastly, damage to the sensorimotor area of the brain will influence motor function. The sensorimotor area includes a vast amount of processes, 
but the primary function is to process and weight afferent sensory data that is then transferred to the basal ganglia and motor cortex to produce the proper efferent response (Murray, et al., 2014). Damage to this area of the brain could influence somatosensory, vestibular, and visual contributions to the maintenance of postural control and locomotion (Alexander \& Crutcher, 1990; Murray, et al., 2014).

Overall, it is unlikely that a concussion injury will influence or damage basal ganglia structure because of the deep central location of the basal ganglia, yet the basal gangliathalamocortical circuits could be interrupted. This interruption, whether it was caused by cerebral edema, axonal shearing, or a brain lesion of frontal/posterior or lateral/medial brain regions, will influence basal ganglia function (directly or indirectly). The inability to excite or inhibit certain cortical structures by the basal ganglia pathways or loops could result in inappropriate motor and non-motor responses (Alexander, DeLong, \& Strick, 1986; Alexander \& Crutcher, 1990). It should be noted that common concussion signs and symptoms typically resolve within a week to two weeks after injury (McCrory et al., 2013). Given the timeline of recovery from a concussion injury, it is speculated that cerebral edema from brain cell bruising is a common problem that contributes to the expressed post-concussion symptoms (McCrory, Davis, \& Makdissi, 2012; McCrory et al., 2013). However, the knowledge regarding basal ganglia function and potential interruption after a closed head injury have not been documented. Further research is needed to indirectly and directly measure the basal ganglia-thalamocortical circuit interruption in the presence of a concussion injury. 


\subsubsection{Vestibular Contributions to Concussion Balance Dysfunction}

Multiple studies state that the dysfunction of the vestibular sensory organs is the primary contributor to observed balance deficits after a concussion injury (Cavanaugh et al., 2005; Cavanaugh et al., 2006; Guskiewicz, 2011; Kiely, 2011; Leddy, Sandhu, Sodhi, Baker, \& Willer, 2012). However, these claims are made using simple static assessments of postural control. In addition, direct measurements of the vestibular sensory organs' damage are not possible. Even with the advancements of brain imaging, direct vestibular organ damage after a concussion injury has not been observed (Slobounov et al., 2012). Thus, indirect assessment of vestibular organ damage is necessary.

The vestibular organs are made up of two major components; semicircular canals and the saccule. The semicircular canals (horizontal, anterior, and posterior) detect angular acceleration changes of the head, while the saccule detects linear acceleration changes (Shumway-Cook \& Horak, 1986). Both components provide spatial location information to vestibular nuclei (Kandel, Schwartz, \& Jessel, 2000). The vestibular nuclei are responsible for the integration of signals from the vestibular organs, spinal cord, cerebellum, and visual system (Kandel et al., 2000). In addition, the vestibular nuclei projects to oculomotor nuclei, reticular and spatial centers, the vestibular regions of the cerebellum, and the thalamus (Kandel et al., 2000). The relationship between the basal ganglia pathways and the contributions from the vestibular nuclei influences on the thalamus cannot be over looked. If damaged, the vestibular organs could provide inaccurate spatial orientation data to the vestibular nuclei, which in turn could provide inaccurate signaling to the thalamus. If inaccurate sensory information is applied to the thalamus via the vestibular organ or nuclei damage after a concussion injury, abnormal postural control could be exhibited. No literature to date has examined or observed direct damage to either the 
vestibular organs or vestibular nuclei. Until direct measurements can occur, indirect measurements must suffice in order to rule out the contributions of the vestibular system to balance dysfunction after a concussion episode.

Benign paroxysmal positional vertigo (BBPV) is a common diagnosis of vertigo, dizziness, and balance dysfunction (Isaradisaikul, Navacharoen, Hanprasertpong, Kangsanarak, \& Panyathong, 2010). No literature exists examining the use of BBPV testing in concussion individuals. Only through simple balance assessments such as the RT or the BESS along the presence of tinnitus, ringing in the ears, have given rise to the belief that vestibular organ damage is the main cause of observed balance dysfunction after a concussion injury (Guskiewicz, 2011). Through the use of BBPV testing such as the Dix-Hallpike maneuver and the Supine Roll test, nystagmus or fast, uncontrollable movements of the eyes could indicate potential damage to the vestibular organs. This test has not been used to assess vestibular contributions to balance dysfunction after a concussion injury. Thus, it is of interest to evaluate whether acute BBPV caused by direct damage to vestibular organs is a significant contributing factor to balance problems after a concussion injury.

Given the lack of data to support the diagnosis of vestibular organ damage after a concussion injury, it is postulated that other mechanisms such as damage to the basal ganglia loops could be the proposed brain mechanism responsible for balance dysfunction after a concussion injury. However, no current methods of postural control assessment are known to directly measure cortical brain function after a concussion injury. It is of interest to evaluate indirect methods, such as gaze behavior and dynamic postural control, along with cognitive assessments of cortical brain function longitudinally. This longitudinal evaluation could provide 
insight into the links between a closed head injury and sensory integration issues following the injury.

\subsection{Return-to-Play Procedure}

The RTP protocol following an athletic concussion is a minimum rest period of 24-48 hours, followed by a graduated return to play protocol once the athlete returns to baseline measures and no symptoms are reported (McCrory et al., 2013). This graduated RTP protocol is a step-wise progression that is dependent upon the self-report of symptoms by the athlete. The first step is no physical or cognitive activity, followed by the second step of light aerobic exercise (walking, swimming, and biking) (McCrory et al., 2005; McCrory et al., 2013). Third is sport-specific exercise such as more complicated drills and skills (McCrory et al., 2005;

McCrory et al., 2013). Fourth, non-contact training drills begin that involve increased coordination and cognitive load (McCrory et al., 2005; McCrory et al., 2013). Fifth, full contact practice can resume with the final sixth step as full return to play (McCrory et al., 2005;

McCrory et al., 2013). Ideally, each step in the graduated RTP protocol should take 24 hours, thus releasing the athlete back to playing in five days. If any post-concussion symptoms or signs are present during the recovery period at any rehabilitation stage, the athlete must return to the prior asymptomatic step, and then attempt to progress from that point (See Figure 4). 


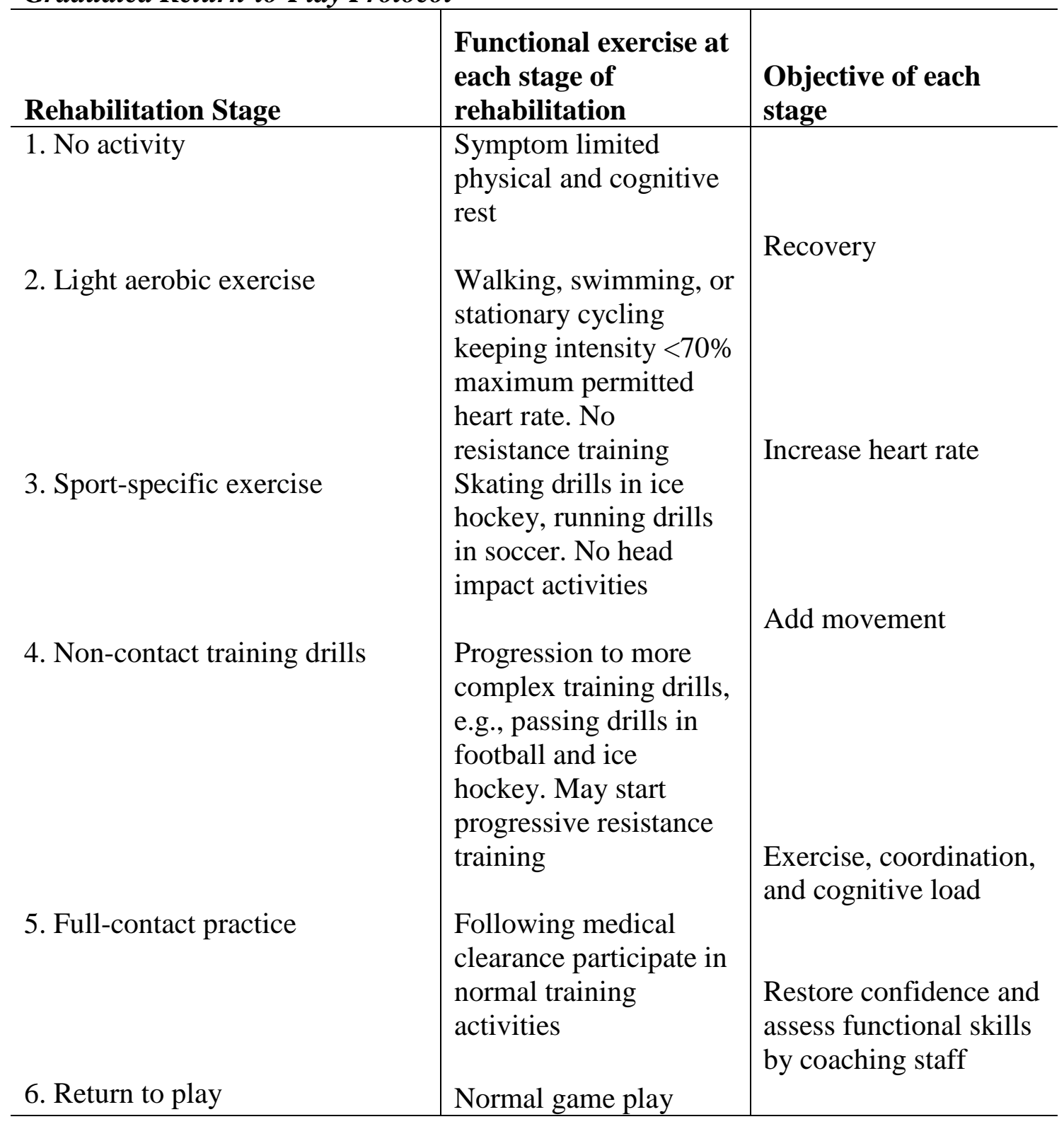

Figure 4: Graduated Return-to-Play Protocol

Note: Protocol set forth by the 4th international concussion consensus statement. Notice the slow rehabilitation process that occurs over time with the given objective of each stage of recovery. Adapted from McCrory et al. 2013. 
No consensus exists on which exercise modalities, beyond the advised functional exercises (Figure 4), are the most effective in the rehabilitation process of RTP following a sportrelated concussion (McCrory et al., 2013). This creates a level of flexibility for each athletic trainer and doctor involved in the management of a sport-related concussion. However, this freedom introduces a lack of consensus and subjectivity on the assessments used to evaluate the progress of recovery. Although the RTP protocol is designed to place the athletes through a series of sport specific drills, the goal of the assessment is to monitor concussion signs and symptoms rather than balance performance. As previously stated, the current balance assessments commonly used to evaluate athlete balance performance and recovery is the RT and the BESS (Guskiewicz, 2011; McCrory et al., 2013). Both the RT and the BESS are low technology assessments of balance that rely upon subjective rater interpretations to evaluate balance and emphasize the maintenance of static balance (Bell et al., 2011; Guskiewicz, 2011; Khasnis \& Gokula, 2003). However, only the BESS balance test is considered reliable and valid for use in sport-related concussion injuries (Bell et al., 2011).

\subsection{TIMELINE OF BALANCE RECOVERY AFTER A CONCUSSION INJURY}

The timeline of balance recovery using the BESS is reported to be 72 hours (Bell et al., 2011; Guskiewicz, Ross, \& Marshall, 2001; Guskiewicz, 2011; McCrea et al., 2005; Riemann \& Guskiewicz, 2000). However, the use of sophisticated force-platforms and visually evoked environmentally relevant dynamic balance assessments report lingering balance dysfunction 1-4 weeks post-concussion (Cavanaugh et al., 2005; Powers et al., 2013; Slobounov et al., 2006; Slobounov et al., 2007). Again, to date no current literature exists examining direct comparisons between static and dynamic balance assessment in the same sample of athletes with concussions. 
Thus given the current gap in the literature, the timeline of balance recovery is inconsistent in the reported literature and needs to be evaluated further in terms of static and dynamic balance assessment methods in the same sample.

\subsubsection{Static Balance Assessment}

Multiple studies have examined the recovery of static balance assessment following a concussion (Bell et al., 2011; Guskiewicz et al., 2001; Guskiewicz, 2011; McCrea et al., 2005; Riemann \& Guskiewicz, 2000). One such study, conducted by McCrea and colleagues (2005), examined a group of 94 football players with concussions and 56 matched controls over one week (7 days) of recovery. Each participant was evaluated with the Graded Symptom Checklist (GSC), Standardized Assessment of Concussion (SAC), and the BESS prior to the respective athletic season beginning and at the same time each day over 7 days of concussion recovery. The results of the study indicated that $36 \%$ of the athletes with concussions had balance impairment, as defined by the BESS. These results were compared to baseline and matched controls, within 24 hours of the initial concussion injury. On day 2 of concussion recovery, only 24\% of injured participants remained impaired on the BESS. After day 3 of concussion recovery, BESS scores for the athletes with concussions group fell within baseline levels. Thus, from this sample the BESS results indicated balance impairment up to 72 hours post-concussion.

An earlier study by Guskiewicz and colleagues (2001) studied 36 collegiate athletes who experienced a concussion and 36 matched controls over five days of recovery. Postural stability assessments were taken prior to the respective athlete's season and at day 1, 3, and 5 of concussion recovery on the Sensory Organization Test (SOT) and the BESS. The SOT systematically disrupts afferent sensory information by reducing spatial awareness cues via 
somatosensory or visual components. Individuals must attempt to maintain a steady, quiet standing posture during six different sensory combination trials. One example of the different sensory conditions is when an individual whose eyes are closed will attempt to remain stable as the force platform translates in the anterior-posterior directions. Another condition is when the environmental cage moves where the force platform does not move. The scoring is a 100 point scale based upon a CoP coordinates, whereas higher scores correlate with better balance as reported by Guskiewicz et al. (2001).

The results from Guskiewicz and colleagues (2001) repeated-measures ANOVA were that the SOT composite scores and BESS scores reported significant differences from one another on days 1,3 , and 5 . In addition, days 1,3 , and 5 were significantly different when compared to matched controls. However, pre-season baseline measurements were not different from the athletes with concussions measurements at day 3 of recovery. These results coincide with McCrea et al., (2005) that postural stability recovered within day 3 as measured by the BESS and the SOT.

Riemann and Guskiewicz (2000) evaluated 16 athletes with concussions and 16 matched control subjects over 10 days of concussion recovery. Each participant was evaluated with the SOT and the BESS to determine recovery of balance post-concussion over the 10 day period. The results of this study indicated that the SOT and the BESS scores showed significant differences on day 3 of recovery when compared to day 1 . However, no differences were observed on day 5 or 10 post-concussion. This study suggests that the balance recovered within day 3 of post-injury based on the results of the SOT and the BESS.

Based on the studies reviewed above, balance dysfunction and impairment as measured by the BESS and the SOT in athletes with concussions subside within 72 hours (3 days) post- 
concussion injury. However, a major limitation of the BESS and the SOT is the purpose of each assessment. Both the BESS and the SOT ask participants to maintain quiet stance regardless of environmental constraints. The emulation of static balance stability is not a sport-specific behavior. Therefore, regardless of the BESS's high reliability (see section 2.1.2) and diagnostic validity evidence (see section 2.1.2) balance dysfunction is not observed after three days postconcussion. Lastly, limitations of the aforementioned studies measured non-replicable methods of CoP and relied on subjective rater interpretations and generated composite scores. Therefore, it is of interest to compare static and dynamic balance assessments in the same sample of athletes with concussions through the use of scientific, reliable force platform data to provide the first direct comparison of two models of assessment during the recovery of concussion.

A recent study by Powers and colleagues (2013) measured static balance deficits in athletes with concussions over the timeline of RTP using a force platform. Nine collegiate football players and nine matched controls (age and position) were examined using a force platform (sampled at $60 \mathrm{~Hz}$ ) to calculate center of pressure measurements during the RTP process. Root Mean Square (RMS) calculations for CoP displacement and velocity were performed for each participant over sixty seconds of two quiet upright stance conditions (eyes open and eyes closed). One evaluation was performed for the matched controls while two evaluation periods were performed for athletes with concussions, acute (24-48 hours postconcussion) and RTP. Return-to-play times differed by each athlete with a concussion, but they ranged from 15-48 days post-concussion. The results of separate mixed model ANOVAs suggest balance dysfunction at the acute phase of recovery in the anterior-posterior RMS CoP displacement. Furthermore, lingering balance dysfunction in the anterior-posterior (acute and RTP time-period) and medial-lateral (acute only time-period) RMS CoP velocity in the eyes 
closed conditions were observed when compared to controls. No differences were determined in any of the eyes open conditions. These results indicate that in the maintenance of quiet stance balance dysfunction was not fully recovered at the RTP measurement period as evidenced by increased velocity of CoP. However, these results were only observed in eyes closed conditions. These results potentially support vestibular system dysfunction as a result of the reported postural instability. By removing the visual system, the vestibular system is than primarily responsible for postural control adjustments (Nashner et al., 1982; Winter, 1995; Winter, Patla, Ishac, \& Gage, 2003). Given the lack of findings in the eyes open conditions, vestibular system dysfunction is suspected. The major limitation of this study is the lack of comparable dynamic game-like conditions in the same sample.

\subsubsection{Dynamic Balance Assessment}

Advances in the science of balance assessment, such as dynamic assessments, may contribute to an understanding of the functional deficits associated with a concussion, which cannot be assessed with traditional static balance assessments. Evidence suggests that damage from a cerebral concussion be functional rather than structural (Lovell, 2009; Shaw, 2002). This functional disruption is observed in the interruptions of neurometabolic functions, known as the metabolic cascade, such as abrupt neuronal depolarization and changes in glucose metabolism (Blennow et al., 2012). These disruptions in neurometabolic function could adversely affect the brain's ability to further excite or inhibit certain structures of the brain involved in the maintenance of postural control. More specifically, the feed-back and feed-forward signals to the areas of the brain responsible for postural control could be interrupted. The manifestation of these postural control errors, as a result of interruptions in neurometabolic brain function, could 
be measured in greater while during challenging functional situations. These situations employ a greater amount of neurological stress. As such, greater functional deficits of the brain could be observed in the evaluation of functional game-like dynamic balance assessments. Thus, it is the natural progression of research in balance assessment after a concussion injury to examine the implications of functional brain deficits through dynamic or functional balance post-concussion.

Functional assessment of postural control should stress all three sensory systems; the visual system, the vestibular system, and the somatosensory system (Horak, 1987). Virtual reality is used as safe and effective tool for recreating functional balance activities. Researchers in concussion management have demonstrated that with the use of virtual reality simulations and dynamic gait assessments, lingering residual balance dysfunction is reported beyond the traditional 72 hour mark (Catena, van Donkelaar, \& Chou, 2009; Parker, Osternig, van Donkelaar, \& Chou, 2007; Slobounov et al., 2006; Slobounov et al., 2007; Slobounov, Cao, Sebastianelli, Slobounov, \& Newell, 2008).

One virtual reality study by Slobounov and colleagues (2006) examined postural responses in a moving room in athletes with concussions. A sample of 48 healthy control subjects and 8 athletes with concussions was assessed on day 3, 10, and 30 post-injury. All participants were evaluated with neurocognitive assessments to confirm the diagnosis of a concussion. Furthermore, participants attempted to maintain quiet stance on a force platform (sampled at 150Hz) while viewing a computer generated moving room. The room moved in a forward-backward, whole room, front wall only, side wall only, and whole room roll motion. The results indicate that CoP displacement increased with respect to viewing the stationary room (maintenance of quiet stance) in the athletes with concussions group; however, it was not significant from days 3-30 of the evaluation when compared to baseline measures. Differences 
were observed in the CoP displacement at day 10 and day 30 of evaluation in the presence of the front moving room only and the roll moving room when compared to baseline measurements. The results suggest that residual balance dysfunction is still present until day 30 of postconcussion recovery in visual field motion.

An expansion of this work in Slobounov and colleagues (2007) compared the differential rate of recovery in athletes after first and second concussion episodes. One hundred and sixty collegiate athletes were tested pre-season in a virtual reality setting similar to the SOT. Of these baselines, thirty-eight experienced a concussion injury and were evaluated with the same methods over days 10,15, and 30 post-concussion. A center of pressure time series was conducted and measured for thirty seconds using a force platform (sampled at 150Hz). Additionally, whole body motion was measured using Flock of Birds motion tracking sensors while participants attempted to maintain quiet stance during the different visual conditions. From this data, a significance and magnitude of coherence (measure of linear dependency or coupling of two signals at a specific frequency) of postural control was calculated and analyzed further. The higher the magnitude of coherence indicates a greater ability of oneself to adjust ones visualkinesthetic (perception and modification of self-motion or postural control) integration in response to a visually evoking environments. The results of this study report that a lower magnitude of coherence at day 10 and 17 of concussion recovery when compared to baseline measurements. This study indicates that athletes with concussions had a lingering balance dysfunction, inability to properly modify postural control in response to the perception of selfmotion, at days 10 and 17 of recovery. However, a limitation of this study is the goal of maintaining quiet stance. As previously stated, maintenance of quiet stance is not a common replicable game-like behavior. Whereas dynamic goal oriented tasks in a constantly changing 
environment emulate sport-specific skills. Expansions of this work should emphasize the use of dynamic balance assessments in order to emulate sport-specific skills.

Gait stability has long been used to evaluate dynamic motor performance in varying clinical populations (Catena et al., 2009; Parker et al., 2007). Dynamic motor performance is the ability to maintain bipedal upright stance or gait during a dynamic task. Gait stability requires one to maintain upright forward progression of one's center of mass (COM) within the limits of stability during forward progression (gait). If one’s COM falls outside the limits of stability a potential fall could occur. Gait stability is a functional daily activity of living and represents an environmentally relevant dynamic motor performance task.

Earlier work by Parker and colleagues (2007) measured 29 athletes with concussions and 29 healthy matched controls over twenty-eight days of recovery. They measured whole-body $\mathrm{COM}$ and CoP directions during a single task and dual task gait cycle. The dual task that participants performed was counting backwards or spelling five-letter words while walking in a straight direction. The influence of dual tasking, such as counting backwards or spelling fiveletter words, places additional cognitive load on participants. This additional load is postulated to interfere with proper sensory integration. In addition, by giving a secondary task during gait it emulates divided attention (gait and cognitive task combined) that could be required of an athlete during a sporting event. For example, a quarterback in American football during a play must know who and when to throw the football while dodging other players.

The results of the study indicate that significant differences were noted in the dual-task gait assessment (slower COM velocity, smaller COM-CoP separation distance, and greater COM sway) in the athletes with concussion group until day 28 of the assessment when compared to the control group. These results indicate that when athletes with concussions are given dynamic 
dual-task and goal oriented behaviors, residual motor dysfunctions are present until four weeks of concussion recovery.

A similar study by Catena and colleagues (2009) measured gait with a concurrent cognitive task in 30 athletes with concussions and 30 matched controls. Using the same methods as reported in Parker et al., (2007) athletes with concussions were evaluated within at days 2, 6, 14, and 28 of post-injury recovery with three walking tasks; (1) single-task level walking, (2) attention divided walking, and (3) obstacle-crossing task. Balance control during gait was measured with whole-body COM and CoP motion. The findings of this study suggest that a divided attention task is able to differentiate between the matched controls and the athletes with concussions until day 6 of recovery. However, obstacle avoidance did not demonstrate significant reductions in gait parameters between the groups until day 14 of recovery. At day 14 of recovery a more conservative approach to stepping over obstacles (reduced medial-lateral velocity) was observed. These results further support that in the presence of a secondary task during a dynamic movement a reduction in motor control function in athletes with concussions is present.

Returning to environmentally relevant dynamic postural control, a recent study by Murray and colleagues (2014) examined the differences in oculomotor control between 9 athletes with concussions and 9 healthy athletes during an active, dynamic balance task. This study measured the amount of eye deviations and the game score on the WiiFit Soccer Heading Game during concussion recovery. The WiiFit Soccer Heading Game provides a unique dynamic virtual environment that introduces a secondary task, obstacle avoidance and interaction, along with the proper maintenance of upright stance. Although researchers often speculate about the 
validity of the WiiFit, it can provide a safe cost effective dynamic game-like game that emphasizes the maintenance of dynamic postural control.

The results of this study report that within 24-48 hours post-injury gaze deviations were twice the amount of the control participants. Furthermore, the WiiFit Soccer Heading Game score was not significantly different over two weeks of recovery in the athletes with concussions. However, control participants demonstrated a significant learning effect on the game. These results suggest that residual postural control dysfunction is still present until two weeks of recovery post-injury with an interruption in the vestibular-ocular reflex of athletes with concussions within 48 hours of recovery. The major limitation of the study was the lack of gaze measurements over the two weeks of recovery. Yet, this study supports that, in the presence of an environmentally relevant dynamic balance task, residual balance dysfunction is observed during concussion recovery.

\subsubsection{Refinement of the Timeline of Balance Recovery}

The reported timeline of balance recovery is inconsistent in the literature. The timeline of balance recovery changes with either static or dynamic balance assessments. These changes could be due to the lack of replicable methods between studies or the variable nature of a concussion injury. In static balance assessments or the maintenance of quiet stance without the presence of visually evoked stimuli, balance recovery return to baseline function within 72 hours post-injury (Guskiewicz et al., 2001; McCrea et al., 2003; Riemann \& Guskiewicz, 2000). However, using sophisticated biomechanical assessments of postural stability in the absence of visual information or eyes closed, residual balance dysfunction is observed until 30 days postinjury (Powers et al., 2013). Further, an environmentally relevant dynamic assessment of 
postural control observes dysfunction in athletes with concussions that is present until 30 days post-injury (Slobounov et al., 2006; Slobounov et al., 2007). Using gait analysis and dual task cognitive loading postural control dysfunction was observed in athletes with concussion until 28 days post-injury (Catena et al., 2009; Parker et al., 2007). Following a dynamic functional gamelike balance assessment, the WiiFit Soccer Heading Game, residual balance dysfunction is observed at two weeks post-injury (Murray et al., 2014).

The range of concussion recovery stretches across 3 to 30 days with no clear consensus reported in the literature. However, the use of environmentally relevant dynamic balance assessments, a true measure of postural control, could be more effective in accurately measuring balance recovery over time. The use of sophisticated scientific measurements that can evaluate the sensory systems involved in controlling postural control, such as the movement of center of pressure, could aid in refining the timeline of balance recovery. Furthermore, it is warranted to evaluate and compare static and dynamic measures of postural control in the same sample of athletes with concussions and controls over the timeline of balance recovery. However, prior to a longitudinal study, further investigation is warranted to investigate changes in postural control during a traditional quiet stance balance task and an environmentally relevant dynamic balance task during one week of recovery in athletes with concussions. A preliminary evaluation of recovery could aid in the quantification of environmentally relevant dynamic balance tasks when compared to traditional quiet stance techniques within the same sample. As previously noted, this has not been performed in the literature to date. Therefore, it is important to compare the sensitivity of a common method of static balance assessment to a dynamic experimental procedure in athletes with concussions prior to longitudinal evaluation. This information is 
critical in refining the RTP guidelines and opening new lines of research in balance assessments that could potentially lead to mitigating the presence of a secondary concussion.

\subsection{Reliability ANd VAlidity EVIDENCE of BaLANCE AsSESSMENTS}

\subsubsection{Reliability and Validity Definitions}

Reliability and validity are critical aspects that must be considered when examining if a measurement tool or test can be used during the evaluation of a particular population. For the purpose of this literature review, reliability and validity operational definitions need to be defined. Reliability is considered the ability of a test to repeat the results of that test over time (Looney, 2006). Reliability coefficients, specifically inter rater and intra rater, that range from 0$1(0=$ no reliability, $1=$ perfect reliability $)$ are the most common methods of reliability reported in allied health science research (Looney, 2006). Thus, reliability coefficients that follow the aforementioned conditions were examined to explore each test or measurement tools ability to reliably be used in concussion management.

Validity reflects the ability of a test to be accurate and to determine if the test measures what it is supposed to measure (Looney, 2006). There are many forms of validity; however, diagnosis validity is critical in the evaluation of injury or disease. Correct clinical diagnosis relies upon the ability of a test to indicate if one has or doesn't have the condition (Looney, 2006). Sensitivity (true positive) and Specificity (true negative) are the statistical indexes commonly used to describe the diagnostic ability of a test or tool within the allied health sciences (Looney, 2006). Therefore, for the purpose of this literature review, diagnostic validity of each test or measurement tool will be examined to determine the likelihood that one has or does not have a balance or cognition impairment after a concussion injury. 


\subsubsection{Balance Error Scoring System}

The BESS was developed by individuals at the University of North Carolina Chapel Hill to provide a low technology, cost effective, sport-related concussion assessment for balance (Guskiewicz, 2011). This test uses a foam platform and a stopwatch and is easily usable as both a sideline and a clinical test. Individuals perform a series of stances that include double, single, and tandem (legs in a heel to toe formation) leg support. The starting and reference position for grading of balance errors is where individuals place their hands on the iliac crest and close their

eyes. A twenty-second clinical observation period begins once the subject's eyes are closed. The subject's performance is assessed by the number of deviations or errors from the original position with a possible maximum of ten errors per trial. Two trials are assessed for each foot position, thus resulting in six total trials.

The BESS has been widely accepted by researchers as an applicable test for assessing balance deficiencies in athletes with concussions (Bell et al., 2011). The BESS has been thoroughly examined and systematically reviewed in many populations, including young adults, old adults, athletes of different sports, and concussion; furthermore, it is emerging as the current gold standard in non-laboratory settings to evaluate balance deficits (Bell et al., 2011). Its reported ICC of the inter-rater reliability between the BESS and more complicated measurements such as the SOT were found to range between 0.78 to 0.96 (Riemann \& Guskiewicz, 2000). Furthermore, when evaluating balance in individuals with a concussion, the sensitivity values for the BESS assessed at baseline are 0.34 , while the specificity values ranged from 0.91 to 0.96 across the first through seventh days of the initial concussion injury (McCrea et al., 2003). Though the BESS has wide popularity and has been clinically validated, limitations remain. Due to the low sensitivity statistics (0.34) and inability to detect balance issues after day seven of the 
initial concussion injury, the BESS is best used as a pre-screening test to help sideline measurements of a suspected concussion (Guskiewicz, 2011). Lastly, the BESS is not an environmentally relevant dynamic balance test that simulates a game like behavior.

\subsubsection{The Romberg Test}

The RT was initially developed, in the late $19^{\text {th }}$ century, to detect sensory impairments in individuals with tabes dorsalis (Lanska \& Goetz, 2000). The purpose of the RT is to assess balance when individuals experience reduced visual sensory input. With reduced visual input, reliance on the vestibular and somatosensory systems for balance and postural modulations will be exaggerated. Commonly used by physicians and other healthcare professionals, the RT evaluates potential balance impairments after a neurological incident such as a concussion (Guskiewicz, 2011; Khasnis \& Gokula, 2003). Postural control can be examined via the RT by the individual standing as quietly as possible without deviating from the standing position. This standing position is where the feet are placed together (medial malleolus touching medial malleolus of each foot) or heel to toe position, with differing visual sensory conditions (Guskiewicz et al., 2001; Ingersoll \& Armstrong, 1992; Riemann \& Guskiewicz, 2000). The visual sensory conditions include eyes open, and eyes closed (Guskiewicz et al., 2001; Ingersoll \& Armstrong, 1992; Riemann \& Guskiewicz, 2000). A positive Romberg sign is given if the individual being tested sways involuntarily during the reduced vision condition (Khasnis \& Gokula, 2003). If abnormal movements or sway are detected during the RT, then it is deduced that a neurological deficit exists within the higher functioning sensory systems (Lanska \& Goetz, 2000). It has been suggested that the sensory dysfunction (associated with tabes dorsalis or concussion) can be related to myelopathies or neuropathies of the sensory system (Khasnis \& 
Gokula, 2003; Lanska \& Goetz, 2000). If myelopathies, neuropathies, or short term reduction in the flow of sensory information to higher brain centers exist, a reduction in balance or postural control will be demonstrated.

Reliability and validity data of the RT is limited. No known published reliability or validity data currently exists for individuals who have experienced a concussion injury. However, reliability evidence does exist for the RT in other neurological dysfunctions. The Testretest Intra-Class Correlation (ICC) for individuals with Parkinson's disease was 0.84 in the reduced vision condition and 0.86 in the normal vision condition (Steffen \& Seney, 2008). These ICC values quantify the reliability of the RT as excellent in individuals with Parkinson's disease.

A potential relationship exists between the sensory deficits observed in a concussion injury and Parkinson's disease. Specifically, both concussion and Parkinson's disease are associated with a reduction in the capacity of the basal ganglia to process sensory input, resulting in insufficient sensory integration and aberrant motor control for postural maintenance (Kandel et al., 2000). However, the comparison between a concussion injury and Parkinson’s disease is highly speculative. Regardless of the similarities in the mechanism underlying postural instability, no reliability or validity data currently exists for the use of the RT in the assessment of concussion injuries (Guskiewicz et al., 2007).

Over the past years, the RT has evolved due to its subjectivity (Jacobson et al., 2011). The Romberg Test of standing balance on firm and compliant support surfaces (RTSBFCSS) is more commonly used for the identification of vestibular dysfunction (Jacobson et al., 2011). The RTSBFCSS expands the traditional standing RT with the addition of a compliant support surface similar to the BESS (Guskiewicz, 2011; Jacobson et al., 2011). The addition of the compliant 
surface emphasizes reliance upon the proprioceptive or vestibular sensory systems, effectively testing the sensory systems most commonly responsible for balance dysfunction.

The sensitivity and specificity values of the RTSBFCSS reported in individuals with vestibular dysfunction were 0.55 and 0.64 , respectively. In older individuals (over the age of 40 years) the sensitivity and specificity values were 0.61 and 0.58 respectively (Jacobson et al.,

2011). Thus, the RT accurately detects balance impairments in approximately $60 \%$ of cases and should not be used as a screening measure for vestibular impairment (Jacobson et al., 2011).

Biomechanical researchers attempt to replicate the RT with more sophisticated force platforms. By having the subjects attempt to maintain quiet stance on different surfaces, researchers can ascertain with greater sensitivity the muscular and motor control contributions to the RT. It should be noted that although researchers are attempting to replicate the RT, the term quiet stance is used more frequently. In addition, no reliability or validity data currently exist for the use of the modified RT or quiet stance measurements in athletes with concussions. However, quiet stance measurements on a force platform are considered the cold standard for postural control evaluations, which include athletes with concussions (Guskiewicz, 2011).

\subsubsection{The WiiFit}

The WiiFit balance game uses visual stimuli to transpose lower body ground reaction force data to meet a certain criteria of goals based upon the game's alternative requirements. The WiiFit has been demonstrated to be a reliable measure for static standing balance assessments of center of pressure when compared to the gold standard laboratory grade force platform (Clark et al., 2010) The ICC was determined to be 0.94 for within the device and 0.89 between the WiiFit and an AMTI force platform (Clark et al., 2010). Furthermore, the WiiFit balance game has been 
demonstrated to improve anterior-posterior sway during quiet stance in older adults (Clark et al., 2010).

The WiiFit is used to assess balance because it was recently adopted by the NCAA to assess balance recovery during a concussion episode in athletes (NCAA sports medicine handbook.2009-2010). Furthermore, WiiFit games such as the basic balance test and the Soccer Heading Game have been reported to relate to functional measures of health (Yamada et al., 2011), and the Wii Balance Board has been determined as a comparable measure of static CoP (Clark et al., 2010; Holmes, Jenkins, Johnson, Hunt, \& Clark, 2012), and is currently used throughout rehabilitation clinics as a rehabilitation and measurement tool. Though the video game has its measurement limitations (limited evidence of reliability and validity for use in concussion assessment), it does represent a comprehensive balance activity involving directing center of pressure in response to visual stimuli.

Examining the WiiFit in Parkinson's disease could provide additional insight into the use of reliability and validity data for athletes with concussions. In a comparable static standing condition, assessments of center of pressure revealed ICCs ranging between 0.92 and 0.98, when comparing a force platform with the WiiFit (Holmes et al., 2012). This evidence further supports the suggestion that the WiiFit can be used as an effective measure of static balance and is comparable to the gold standard of a force platform (Clark et al., 2010; Holmes et al., 2012). However, the WiiFit may be less accurate during active balance tasks that involve sudden dynamic movements.

In participants with lower extremity injury, the WiiFit had poor concurrent validity relative to center of pressure $(\mathrm{CoP})$ and Star Excursion Balance Test (SEBT) measurements (Wikstrom, 2012). During active movements, the Inter-Session Correlation Coefficient (ISCC) 
ranged from 0.39 to 0.80 , while the intra-session reliability scores ranged from 0.29 to 0.74 (Wikstrom, 2012). Given the large ranges of reliability scores during active movements, it can be suggested that WiiFit exhibits poor reliability and validity in individuals with lower extremity injury and in dynamic movements. However, a major limitation of this study was the use of lower extremity impaired individuals. This limitation limits the generalizability of this study.

There is a wide range of WiiFit games. Each game requires and challenges a variety of motor control strategies to accomplish the pre-determined goals of the game (Michalski et al., 2012). Therefore, it is essential for researchers to identify which type of WiiFit game would appropriately assess balance. In community dwelling older adults, dual task walking and dual task timed up and go have been significantly correlated to the WiiFit basic step test (Yamada et al., 2011). The WiiFit basic step test is a replication of the basic aerobic step test, which is considered a functional health assessment for submaximal and maximal cardiorespiratory fitness (Howley \& Thompson, 2012). Thus, the WiiFit is currently being used in fitness and rehabilitation clinics to asses and aid in recovery from injury. However, the validity and reliability measures of balance assessment in individuals with concussion have not been established for the WiiFit. Additionally, prior research has presented that no relationship exists between the WiiFit and the other standard clinical assessments of balance in older adult populations (Reed-Jones, Dorgo, Hitchings, \& Bader, 2012). However, this has yet to be documented during postural control assessments after a concussion.

\subsection{RELIABILITY AND VALIDITY EVIDENCE OF THE IMPACT TEST}

The Immediate Post-Concussion Assessment and Cognitive Testing (ImPACT test) is currently the main tool to aid in the assessment of RTP decisions for athletes who experience a 
concussion (Covassin, Elbin III, Stiller-Ostrowski, \& Kontos, 2009; Salvatore \& Fjordbak, 2011). Given the cost of a functional Magnetic Resonance Imaging (fMRI) or Computed Tomography (CT) scans, along with the insensitivity to detect and identify cognitive impairment after a concussion episode, the ImPACT test is the clinical tool of choice in the mediation of the RTP process (Covassin et al., 2009; Lovell, 2006; Salvatore \& Fjordbak, 2011). The ImPACT Test is a computer based neuropsychological test that measures different components of cognitive functioning (Lovell, 2006). The ImPACT test has high reliability (Interclass Correlation of 0.96 ) and high validity, Sensitivity of 0.65 and Specificity of 0.81 in predicting recovery from a concussion episode (Lau, Collins, \& Lovell, 2011; Lovell, 2006).

However, the ImPACT test is a computer based non-functional assessment. Thus, the relationship between the ImPACT test and other functional measures of health, specifically balance, is a research consideration. It can be postulated that the ImPACT test could be used to point to the potential brain mechanisms involved in the motor processes of specific body functions, such as balance. To date it is unclear whether cognition or motor control is related neurologically. However, through the use of the ImPACT test, cognitive interruption and recovery can be reliably measured after a concussion injury (Lovell, 2009). Yet the ImPACT test's major flaw is its lack of concurrent validity to functional measures of health, such as balance assessments like the BESS. This area of concussion research, using the ImPACT test to point to potential motor processes, has yet to be fully investigated. 


\section{CHAPTER 3: METHODOLOGY AND RESEARCH APPROACH}

\subsection{RESEARCH SETTING}

Two groups of athletes were evaluated over time in this cohort study design (Park \& Schutz, 2006; Shadish, Cook, \& Campbell, 2002; Sullivan, 2012). This design allows one to evaluate the gathered data within and between each cohort over time prospectively. The cohorts in this study were athletes with concussions and athletes without concussions. The time aspect of the study design was to monitor recovery of balance in athletes with concussions compared to healthy controls during recovery.

All participants were assessed at the CMC and the Virtual Reality and Motor Control Research Lab located within the Campbell Building on the campus of UTEP. Athletes with concussions reported to the $\mathrm{CMC}$ for initial evaluation and completion of the assessments weekly. Healthy participants reported to the Virtual Reality and Motor Control Research Lab for testing weekly. All balance assessments were conducted in the Virtual Reality and Motor Control Research Lab for all participants over the entire evaluation period.

\subsection{PARTicipants}

Twenty non-active (10 males; 10 females; mean age $23.8 \pm 3.76$ ), or athletes not currently participating in an NCAA division I sport season, and eleven (8 males; 3 females; mean age $20.08 \pm 1.44$ ) athletes with concussions participated in this study. Non-active or not currently participating in a sporting season athletes were chosen due to the inability to mitigate subconcussive hits during the respective season. A total sample size of 22 (11 in each group) was deemed sufficient in order to meet the desired power of this study (see section 3.5.6 Power Analysis). 
Control participants and athletes with concussions were recruited by the principal investigator at the Concussion Management Clinic (CMC) at the University of Texas at El Paso (UTEP). All participants satisfied the inclusion and exclusion criteria for this study (see Table 1 and Table 2).

Table 1: Inclusion and Exclusion Criteria of Healthy Control Participants

\begin{tabular}{|c|c|}
\hline Inclusion & Exclusion \\
\hline Ages 18-30 & $<18,>30$ years of age \\
\hline $\begin{array}{l}\text { Physically active ( }>10 \text { hours a } \\
\text { week) }\end{array}$ & $\begin{array}{l}\text { Involved in a current NCAA sanctioned } \\
\text { athletic sporting season }\end{array}$ \\
\hline Free of Musculoskeletal Injury & Prior head trauma within the past 12 months \\
\hline Free of Neuromuscular injury & $\begin{array}{l}\text { Documented concussion within the past } 12 \\
\text { months }\end{array}$ \\
\hline $\begin{array}{l}\text { No traumatic brain injury within } \\
\text { the past } 12 \text { months }\end{array}$ & $\begin{array}{l}\text { Loss of consciousness within the past } 6 \\
\text { months (related or not related to a head } \\
\text { injury) }\end{array}$ \\
\hline No history of psychiatric illness & History of Seizures \\
\hline $\begin{array}{l}\text { No diagnosis of learning } \\
\text { disorder or ADHD }\end{array}$ & $\begin{array}{l}\text { Admitted to the Hospital on suspicion of a } \\
\text { head injury }\end{array}$ \\
\hline No history of seizures & $\begin{array}{l}\text { Seven or more total symptoms in any } \\
\text { symptom clusters PCSS of the ImPACT test }\end{array}$ \\
\hline $\begin{array}{l}\text { Less than seven total symptoms } \\
\text { in any symptom clusters of the } \\
\text { Post-Concussion Symptom } \\
\text { Scale (PCSS) of the ImPACT } \\
\text { test }\end{array}$ & $\begin{array}{l}\text { Reporting of any symptoms in the general } \\
\text { healthy questionnaire and interview }\end{array}$ \\
\hline $\begin{array}{l}\text { No symptoms reported in the } \\
\text { general healthy questionnaire } \\
\text { and interview }\end{array}$ & \\
\hline
\end{tabular}


Table 2: Inclusion and Exclusion Criteria for Athletes with Concussions

\begin{tabular}{l|l}
\hline \multicolumn{1}{c|}{ Inclusion } & \multicolumn{1}{c}{ Exclusion } \\
\hline Ages 18-30 & $<18,>30$ years of age \\
$\begin{array}{l}\text { Physically active (>10 hours a } \\
\text { week) prior to injury }\end{array}$ & Prior head trauma within the past 12 months \\
$\begin{array}{l}\text { Free of Musculoskeletal Injury } \\
\text { mocumented concussion within the past } 12\end{array}$ \\
$\begin{array}{l}\text { Diagnosed Concussion by a } \\
\text { medical doctor or athletic } \\
\text { trainer }\end{array}$ & $\begin{array}{l}\text { Loss of consciousness within the past } 6 \\
\text { months (not related to the concussion injury) }\end{array}$ \\
$\begin{array}{l}\text { Free of Neuromuscular injury } \\
\text { other than a diagnosed disease }\end{array}$ & $\begin{array}{l}\text { History of seizures (prior to or since the } \\
\text { concussion injury) }\end{array}$ \\
$\begin{array}{l}\text { No history of psychiatric illness } \\
\text { Reporting of any symptoms in } \\
\text { the general healthy } \\
\text { questionnaire and interview } \\
\text { test }\end{array}$ & $\begin{array}{l}\text { No symptoms in the migraine or } \\
\text { neuropsychiatric PCSS cluster of the } \\
\text { ImPACT test }\end{array}$ \\
$\begin{array}{l}\text { No diagnosis of learning } \\
\text { disorder or ADHD } \\
\text { or since the concussion injury) }\end{array}$ & $\begin{array}{l}\text { No symptoms reported in the general healthy } \\
\text { questionnaire and interview }\end{array}$ \\
\end{tabular}

All participants were physically active but considered non-active or not currently participating in a sport season activity (e.g. football, hockey, strength and conditioning, volleyball, track and field, and the like) for a minimum of 10 hours per week. Healthy control participants were chosen at random from all individuals who responded, and meet the inclusion 
criteria, to an email sent out to the student body at UTEP, classroom visits, and face-to-face interactions. The classroom visits were to eight different Kinesiology courses at UTEP. All healthy participants were free of any musculoskeletal, neuromuscular, or prior head trauma as determined by the health status questionnaire and patient history form. Prior head trauma is defined in Table 1 and Table 2.

Athletes with concussions had a confirmed concussion by the head athletic trainer at their school of residence and/or medical doctor on staff. Athletes with concussions were referred to the CMC and participated in the study within 48 hours post-concussion. No athletes with concussions reported experiencing an additional concussion injury during the time of testing. In addition, nine athletes with concussions reported a single symptom in the migraine or neuropsychiatric symptom cluster of the Post-Concussion Symptom Scale (PCSS) reported by the ImPACT test (see section 3.4.5 Cognitive and Neurological Assessment: ImPACT Test for further justification). Subsequently, athletes are becoming familiar with the symptoms of a concussion and have a tendency to underreport their symptoms. As such, if any athletes with concussions did not report any symptoms on the PCSS, but reported a concussion symptom in the clinical interview and/or the health status questionnaire and history, they were included in the study. Two athletes with concussions reported no symptoms on the PCSS, however, disclosed multiple symptoms on the general health questionnaire and history. Healthy controls had less than seven total symptoms on the PCSS reported by the participant on the ImPACT test. The PCSS on the ImPACT Test was used as a screening device to ensure that athletes with concussions presented neurological signs and symptoms of a concussion (Iverson, 2007; Kontos et al., 2012; Lau, Collins, \& Lovell, 2012). After the principal investigator explained the procedures of the study, all participants gave their informed written consent to participate in the 
study. The Institutional Review Board for UTEP approved the use of human participant involvement prior to any data collection (Appendix A). Subsequently, all participants completed a health status questionnaire (Appendix B) and a patient health history at the first week of testing (Appendix C).

\subsection{STUDY DESIGN}

As previously stated, two groups of athletes were evaluated over time in this cohort study design (Park \& Schutz, 2006; Shadish et al., 2002; Sullivan, 2012). Healthy participants performed the balance tests twice with 7 days in between measures, thus, resulting in two evaluation periods (Time 1 and Time 2). Healthy participants completed the ImPACT Test at the end of Week 2 testing. Athletes with concussions performed the assigned tasks $24-48$ hours postinjury (Time 1), and at a follow up measurement (Time 2). The follow up measurement days ranged from 4 to 14 days (mean days $=8.19 \pm 3.34$ ). No pre-injury baseline or pretest scores were collected for the athletes with concussions group. No intervention, beyond total cognitive and physical rest for a minimum of 24-48 hours post-injury for the athletes with concussions was employed in this study.

Two independent variables and three dependent variables (see section 3.4.1 Dependent variables and section 3.4.2 Independent variables) were assessed in this study. Random assignment was not conducted in the athletes with concussions group or the control group due to a concussion being an unanticipated event. Thus, the concussion group and control group resulted in a convenience sample. All study participants received an incentive (\$40 gift card to Target, BestBuy, or Applebee’s) after successful completion of all data collections. 


\subsection{VARIABLES}

\subsubsection{Dependent Variables}

- Center of Pressure - normality and order of underlying human movement pattern of balance

o Static - Sixty seconds of quiet standing in eyes open and eyes closed

- Eyes open Peak CoP Velocity (A/P and M/L) directions

- Eyes closed Peak CoP Velocity (A/P and M/L) directions

o Dynamic- Seventy seconds of play on the WiiFit Soccer Heading Game

- Peak CoP Velocity (A/P and M/L) directions

\subsubsection{Independent Variables}

- Time (Two assessment periods -- 1 week of recovery)

- Groups (Control vs. Athletes with Concussions)

\subsection{Procedures}

\subsubsection{Control Group}

Participants were assessed twice with 7 days between all measures, resulting in 2 data collection sessions (Time 1 and Time 2) for each control participant. On each day of testing, balance assessments were randomized and administered from the following list: 1) Quiet stance static eyes closed 2) Quiet stance eyes open and 3) WiiFit Soccer Heading Game. In addition, the participants took the ImPACT test once at Time 2 only. The static balance conditions consisted of three trials of eyes open and eyes closed quiet standing, feet together, on the force platform for 60 seconds. The WiiFit Soccer Heading Game consisted of three trials, each trial lasts 
approximately 70 seconds, of the game. During the first visit, each participant played 4 trials (1 trial of familiarization, with 3 trials of measurement) of the WiiFit Soccer Heading Game. After the first visit, the participants completed 3 rounds of the WiiFit Soccer Heading Game. Simultaneously, CoP coordinates were collected during the WiiFit Soccer Heading Game (see section 3.6.1 Postural Control). All athletes were evaluated independently of one another to remove data bias due to potential competition.

\subsubsection{Athletes with Concussions Group}

Athletes with concussions were evaluated in the same manner as the control group with minor changes. Each athlete with a concussion was evaluated within 24-48 hours of the injury and at a follow up appointment (approximately 8 days between all measurements). The time between follow up appointments varied due to requests made by athletic training staff to evaluate the athletes sooner or later in order to return the athlete to a particular athletic event. In addition, each athlete with a concussion was provided with two minutes of rest between all evaluations. The procedures and order of data collection for the athletes with concussions group were randomized in the same manner as the control group. Lastly, the athletes with concussions took the ImPACT test at each testing session. All athletes were evaluated independently of one another to remove data bias from competition.

\subsection{MATERIALS, MEASURES, AND INSTRUMENTATION}

\subsubsection{Patient history, health status questionnaire, and screening tests}

Participants completed a questionnaire that examined past injuries and overall health of the participants during the first assessment period (see Appendix B: Health Status Questionnaire and Appendix C: History Questionnaire) (Stewart, Hays, \& Ware, 1988). This questionnaire 
evaluated if the athlete was free of any musculoskeletal or neuromuscular disorders. The questionnaire is considered to have high reliability $(0.88)$ and good validity evidence for use in clinical populations including concussion (Stewart et al., 1988). The three page document was filled out by the participant and reviewed by the principal investigator prior to data collection. In addition, participants completed the Dix-Hallpike maneuver and the Supine Roll test to report the existence or absence of benign paroxysmal positional vertigo (BPPV) (see section 3.6.4 DixHallpike Test of Benign Paroxysmal Positional Vertigo). Participants were not eliminated from the study if a positive Dix-Hallpike maneuver or Suprine Roll test sign were identified. Lastly, participant's self-reported neurological symptoms, from the PCSS of the ImPACT test, were collected in order to screen for neurological deficits present or absent after the concussion injury (see section 3.6.5 Cognitive and Neurological Assessment: ImPACT Test). Both the DixHallpike maneuver, the Supine Roll Test, and the ImPACT test were used as screening tests. The results of these tests are documented and reported in the results of this study.

\subsubsection{Postural Control}

Static and dynamic center of pressure (CoP) data were collected by placing the WiiFit Balance Board (WBB) directly over the top of a force platform (AMTI, Watertown, MA, USA) (Figures 6 and 7). Prior to any data collection, the force platform, with the WBB on top of it, was zeroed in order to account for the weight of the WBB and for normalization purposes. Static postural control and steadiness were measured during static quiet feet together standing eyes open and eyes closed conditions over sixty seconds at a sampling frequency of $50 \mathrm{~Hz}$ without shoes. Static postural control calculations investigated Peak CoP Velocity in the A/P and M/L directions and processed by using a custom CoP calculation in MATLAB (2010, MathWorks, 
Inc., Natick, MA, USA). During the static trials, participants were instructed to gaze directly into a white projector screen, located approximately 55 inches in front of the WBB. Dynamic postural control was measured during the WiiFit Soccer Heading Game at a sampling frequency of $100 \mathrm{~Hz}$ without shoes. Dynamic calculations of postural control were Peak CoP Velocity in the A/P and M/L directions and processed by a custom CoP calculation in MATLAB (version 2010, Natick, MA, USA). These methods were employed because they are the common methods for measuring posture in concussion research. Furthermore, force platforms are the gold standard for postural control evaluation (Guskiewicz, 2011).

Any abnormal trials of either static or dynamic CoP were not included in the study. A trial was considered abnormal if the participants took a step, sneezed, or were suddenly distracted by the external environment. The participants were either asked to repeat the abnormal trial. If they declined due to stated or unstated reasons, the abnormal trial was removed at the primary investigators discretion post hoc. Only twelve trials, out of five hundred and eighty, were considered abnormal and were removed from data analysis.

\subsubsection{WiiFit}

Participants performed an environmentally relevant dynamic balance assessment using the Nintendo WiiFit ${ }^{\circledR}$ Soccer Heading Game. The Soccer Heading Game used the WBB to measure CoP and allowed the participant to direct an on-screen avatar via lower body CoP postural adjustments. Participants were awarded points by successfully intercepting and heading a soccer ball while dodging distraction objects. Contact with distracter objects (shoes and/or

panda heads) reduced the participant's score. During the test, participants stood on the WBB (18 inches X 10 1/2 inches) without their shoes, directly in front of a 50 x 50 inches screen, 
approximately 55 inches away from WBB. The room was darkened in order to maintain the projection screen resolution and to remove potential distractions during play. Due to its lack of reliability, validity, and the presence of a learning effect, the WiiFit Soccer Heading Game score was not used in this study. Only CoP directions were recorded and further analyzed. All participants were given a pre-test practice that simulates the balance assessments for familiarization during the first assessment period. Subsequently, on each follow up appointment, each participant completed three trials of the Soccer Heading Game without any practice or warm-up.

\subsubsection{Dix-Hallpike Test and the Supine Roll Test of Benign Paroxysmal Positional Vertigo}

Two assessments of abnormal saccades and smooth pursuit of the eyes were performed using the Dix-Hallpike benign paroxysmal positional vertigo (BPPV) test and the Supine Roll test. These tests are commonly used to determine peripheral vestibular organ damage (Fife et al., 2008). The Dix-Hallpike maneuver is primarily used to assess issues within the anterior and posterior semicircular canals (Fife et al., 2008). The Supine Roll test is primarily a test to assess issues within the lateral/horizontal semicircular canal (Fife et al., 2008). These tests were not included in any statistical analysis nor are they dependent variables; they were employed as a screening test only to report the existence or absence of BPPV for demographic purposes. Participants were not excluded from the study if they demonstrated a positive nystagmus sign on either test.

Following the procedures described by Fift et al. (2008), the Dix-Hallpike test was performed by the primary researcher with the participant sitting on a treatment table. The primary researcher manually turned the participants head to a 45 degree position, in the frontal 
plane, relative to the sternum towards the side of the head being evaluated. Maintaining this position, the primary researcher laid the participant down onto the treatment table to the point where the participant's head is hanging approximately 5 inches below the level of the table. Maintaining the head position, the participant was then returned to the upright position in the same manner as described above. During this entire maneuver, the participants head and trunk position are maintained. In addition, the primary researcher assessed for nystagmus and asked if the participant experienced any dizziness or similar symptoms. A positive nystagmus sign resulted in the eyes rolling from side to side suddenly and without purpose. A negative nystagmus sign resulted in the eyes staying stationary or moving with purpose. This process was repeated for both left and right sides of the head in order to assess left or right side nystagmus. This test is considered a reliable measure of determining vestibular damage; however the test did rely upon rater judgments as to the presence or absence of a positive or negative nystagmus sign (Isaradisaikul et al., 2010). The raters were trained via online tutorials and hands-on demonstrations.

Again, following the procedures described by Fift et al. (2008), the Supine Roll test was performed by the primary researcher with the participant lying supine (face up) with the head in a neutral anatomical position. The head was then manually manipulated by the primary researcher to 45 degree position, in the frontal plane, relative to the sternum. The primary researcher observed the eyes and evaluated the presence of nystagmus while they manipulated the head. A positive nystagmus sign resulted in the eyes rolling from side to side suddenly and without purpose. A negative nystagmus sign resulted in the eyes staying stationary or moving with purpose. This was then repeated twice more to confirm the primary researcher's observations for both the left and right sides of the body. This test is considered a reliable 
measure of determining vestibular damage; however the test did rely upon rater judgments as to the presence or absence of a positive or negative nystagmus sign (Fife et al., 2008).

The reliability of the primary researcher's reports was performed to ensure consistency of the reports. As such, the eyes during each Supine Roll test were visually recorded by a video camera. These recordings were analyzed the following day by two additional trained raters. Each evaluation, including the original rater, by the trained raters was independent of one another. The point-to-point reliability was conducted between the original trained rater's interpretation and the second and third trained raters' interpretation, of the video recording, for the presence of nystagmus. The point-to-point reliability was calculated and is reported in the results of this study. The reliability of the Dix-Hallpike maneuver was not performed given the difficulties of recording the eyes during this complex maneuver without the aid of Frenzel glasses.

\subsubsection{Cognitive and Neurological Assessment: ImPACT Test}

The Immediate Post-Concussion Assessment and Cognitive Testing (ImPACT) was used to detect and quantify cognitive impairment and the symptoms the athletes with concussions experienced after the injury (Covassin et al., 2009). The ImPACT test is a computer based neuropsychological test that measures different components of cognitive functioning (Covassin et al., 2009; Lovell, 2006; Salvatore \& Fjordbak, 2011). Furthermore, participants documented their experienced concussion symptoms during the past 24 hours while taking the test. For the purposes of our study, the ImPACT was a screening test to report the demographic symptoms experienced by the participant. The reported PCSS from the ImPACT test was not a dependent variable. The PCSS that were reported by the participant was used to determine the presence or absence of neurological deficit after a concussion injury. As such the cognitive aspects of the 
ImPACT test were not reported. This is because not all athletes with concussions have cognitive impairments after a concussion injury (Lovell, 2009). In addition, the PCSS reported by participants on the ImPACT test are considered highly reliable and are directly linked to neurological impairments after a concussion injury (Iverson, 2007; Kontos et al., 2012; Lau et al., 2012).

The PCSS is the individual's perception of how they felt within the past 24 hours. The participant reported PCSS were broken down into four clusters: (1) migraine, (2) cognitive, (3) sleep, (4) neuropsychiatric (Iverson, 2007; Kontos et al., 2012; Lau et al., 2012). The migraine cluster includes headaches, visual problems, dizziness, noise/light sensitivity, nausea/vomiting, balance problems, and numbness/tingling. The cognitive cluster includes fatigue, fogginess, drowsiness, difficulty concentrating/remembering, and cognitive slowing. The sleep cluster includes difficulty sleeping, sleeping less than usual, and sleeping more than usual. The neuropsychiatric cluster includes sadness, more emotional, nervousness, and irritability. Participants rated the severity of each symptom using a 7 point Likert scale where 0 indicates the absence of that symptom and 6 indicates the highest severity of that symptom (Lau et al., 2012). The migraine and neuropsychiatric clusters are directly linked to one another and to neurological deficits experienced after a concussion injury (Lau et al., 2012). Furthermore, as our study examined the recovery of balance, it is critical to include any participants that report balance problems, visual problems, headaches, and dizziness. Thus, if any athletes with concussions had a single symptom in either the migraine or neuropsychiatric symptom clusters, as a result of the concussion injury, they were considered to have a concussion injury. These participants were included in our study. Furthermore, the total PCSS symptoms were not necessarily related to a concussion injury. For example, a healthy individual could be experiencing a headache/migraine 
at the time of taking the ImPACT test. As such, from the reported literature our healthy control participants must not exceed a total PCSS of six in any of the symptom clusters (Iverson, 2007). If a healthy control participant reported greater than six total symptoms on the PCSS, they were not included in the study. Lastly, these symptoms further support the concussion diagnosis by the doctor or the athletic trainer.

\subsubsection{Timeline of Balance Data Collection}

The total time that it took participants to complete the balance testing is found in Figure 5. The balance testing did not exceed approximately forty minutes per participant per assessment period.

\section{Static Balance Assessment}

6 total trials@60 seconds with 2

minutes rest $=18$ minutes total

\section{WiiFit Soccer Heading Game}

3 total trials@70 seconds with 2

minutes rest $=9.5$ minutes total

\section{Dix-Hallpike Maneuver and Supine Roll Test \\ 6 total trials@20 seconds with2 minutes rest $=7$ minutes total}

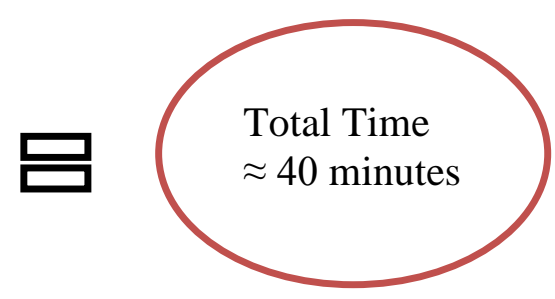

Figure 5: Timeline of balance data collection.

Notes: The total time of the balance assessment, including the rest periods, did not exceed more than 40 minutes. Participants were given additional rest periods as requested or if deemed necessary by the principal investigator. Note: all balance tests were randomized, the boxes for each assessment are not in a specific procedural sequence.

\subsubsection{Experimental Setup}

Figure 6 demonstrates the experimental setup of the static postural control quiet stance assessment. As depicted, individuals attempted to stand in an upright position directly on top of 
the WBB, which was on the force platform. The conditions included three trials of eyes open and eyes closed quiet stance for sixty seconds, resulting in six trials of quiet standing for each assessment period. The participants were evaluated during the two different trial conditions (eyes open and eyes closed) where individuals attempted to maintain quiet stance while standing with their feet together, hands at the side for 60 seconds. During the eyes open conditions, participants were instructed to focus on the white projection surface, while during the eyes closed conditions participants were instructed to simply keep their eyes closed. Figure 7 demonstrates the experimental setup of the dynamic postural control assessments. As depicted, individuals attempted to play the Soccer Heading Game while maintaining upright posture on the WBB with the feet spread at shoulder width apart. Three trials of the WiiFit Soccer Heading Game were conducted during each assessment period.

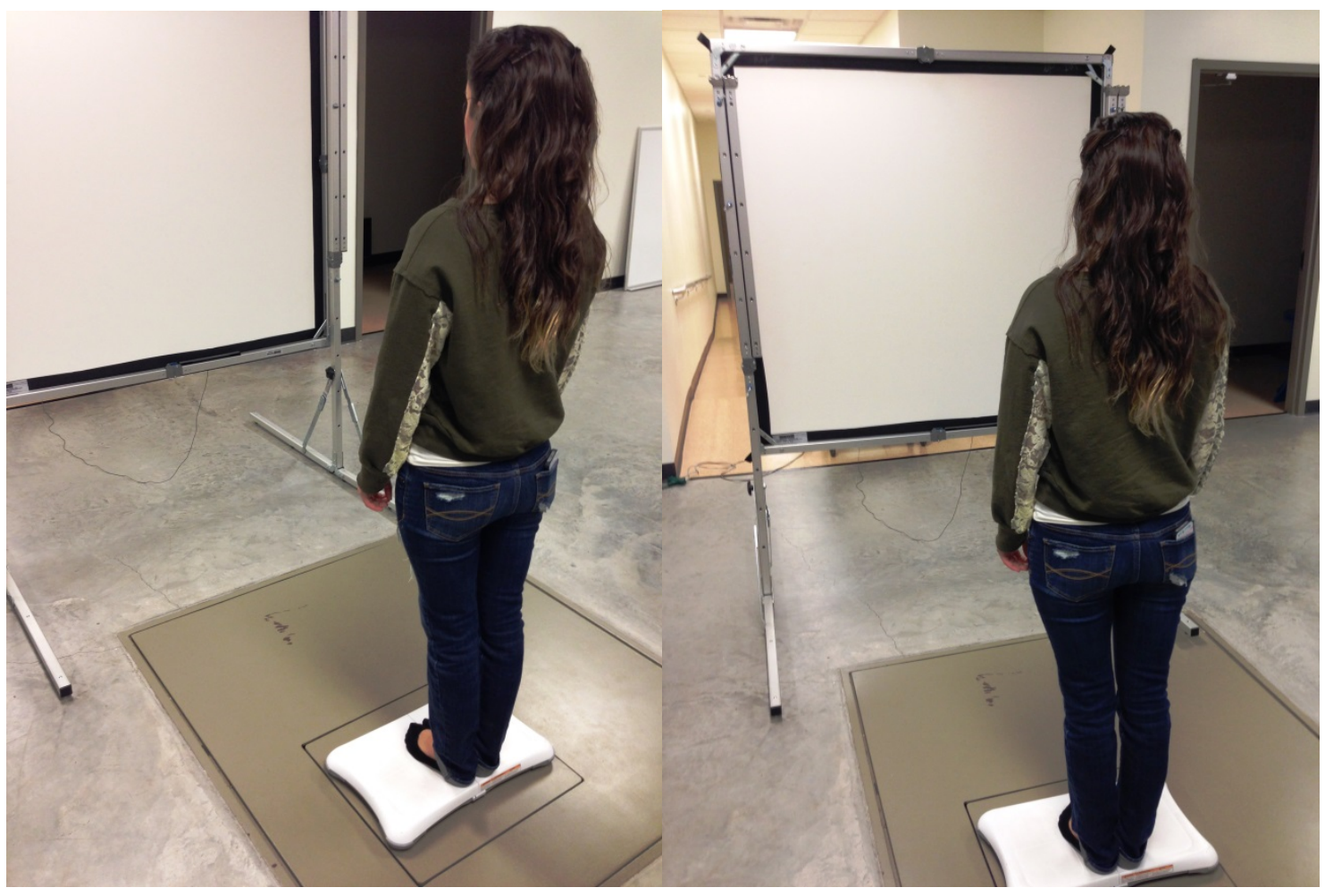

Figure 6: Static postural control assessment. 
Notes: During two different trial conditions (eyes open and eyes closed) where individuals attempted to maintain quiet stance while standing with their feet together, hands at the side for 60 seconds. During the eyes open conditions, participants were instructed to focus on the white projection surface, while during the eyes closed conditions participants were instructed to simply keep their eyes closed. Please note the white Wii Balance Board that is placed directly on top of the force platform.

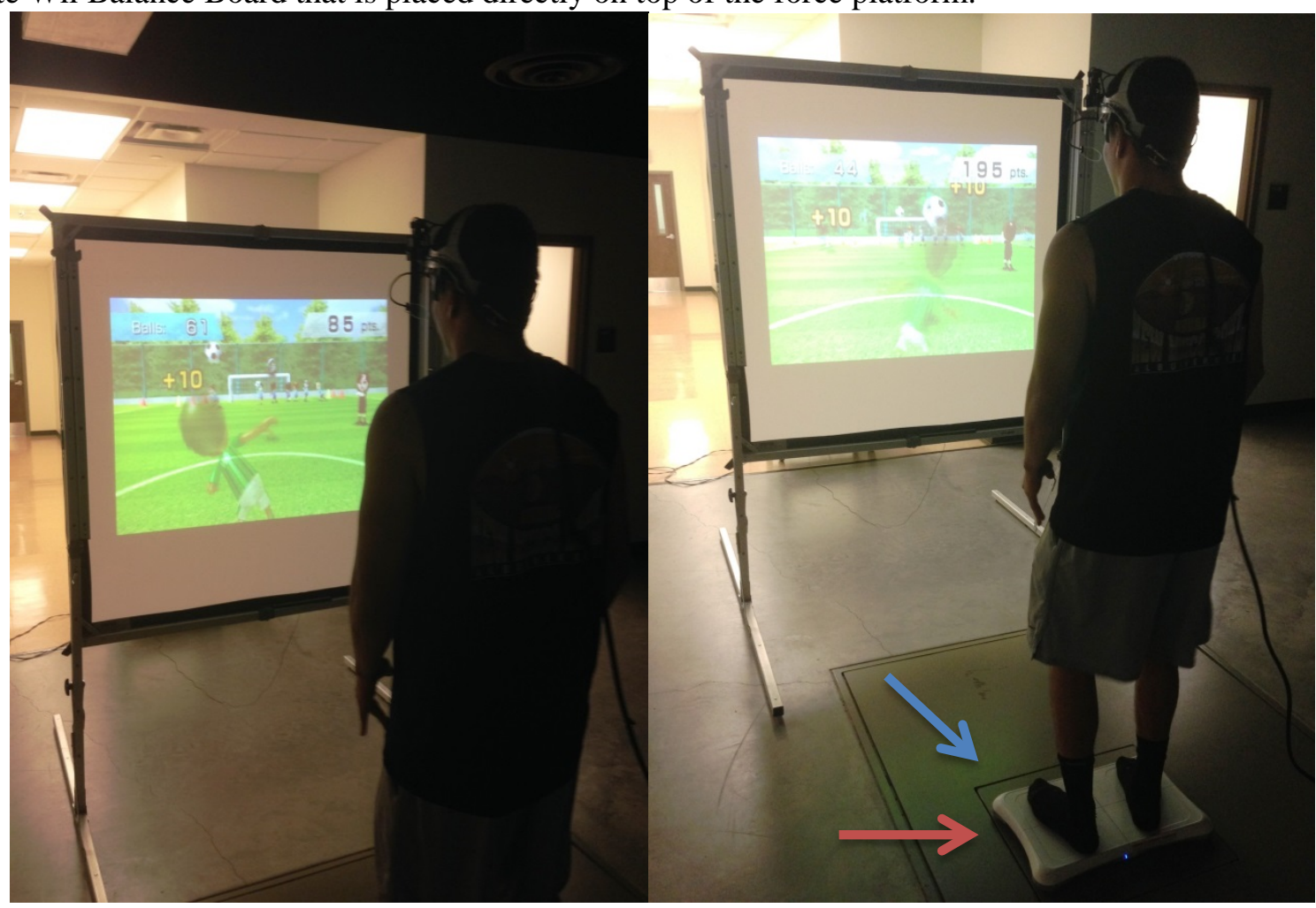

Figure 7: Dynamic postural control assessment the WiiFit Soccer Heading Game.

Notes: Observe that the participant stands (shoulder width apart) on top of the Wii Balance Board that is placed on top of the force platform. The red line is pointing to the Wii Balance Board, while the blue line is pointing to the force platform. As the participant shifted ones center of mass, the avatar moved in the direction of travel. Please note that the attachment on head of the player is a monocular eye tracker that was not included in this study but part of larger ongoing study. The monocular eye tracker does not affect the athletes score on the game (Murray et al 2014).

\subsubsection{Intervention}

In this study, no direct intervention was provided beyond total cognitive and physical rest for a minimum of 24-48 hours following the initial concussion injury. In addition, further total rest could be prescribed by the concussion management clinicians as needed. Of the athletes with 
concussions, a total of 8 athletes with concussions were instructed to maintain total rest until the next evaluation period.

\subsubsection{Statistical Design}

Six mixed model ANOVAs (2 groups x 2 assessment periods) assessed differences for each dependent measure (eyes open Peak CoP Velocity A/P, eyes open Peak CoP Velocity M/L, eyes closed Peak CoP Velocity A/P, eyes closed Peak CoP Velocity M/L, WiiFit Soccer Heading Game Peak CoP Velocity A/P, WiiFit Soccer Heading Game Peak CoP Velocity M/L) to compare post-concussion group to controls during approximately 8 days of recovery. Significant interactions between group and time were further investigated by simple effects tests to compare the group difference at each time, or the time effect for each group. For a significant time effect for the healthy athletes (control group) or athletes with concussions group, follow up one-way ANOVAs were used to test differences in means between the two times. An alpha level of 0.05 was set a priori.

\subsubsection{Power Analysis}

In order to reduce the chances of committing a Type I error, rejecting the null hypothesis when it is true (false negative), prior research means and standard deviations (SD) were consulted in order to determine the appropriate effect size needed for the proposed study. Each dependent variable to be included in this study (see 3.4.1 Dependent Variables) means and SD were collected from prior research. From prior research means, SD, correlations, desired power, and a set alpha level, the effect sizes were calculated in order to determine the appropriate sample size for the study. From Table 3, the effect sizes calculated ranged from 0.49 to 0.97 for all of the dependent variables using a Cohen's $d$ and an effect size correlation calculator (Becker, 
University of Colorado Colorado Springs, 2013). Within the acute phase of concussion recovery (24-48 hours post-concussion) eyes open static CoP effect sizes ranged from 0.54 to 0.97 , and eyes closed static CoP effect sizes ranged from 0.54 to 0.86 (see Table 3). Whereas, at 10 days post-concussion eyes open static CoP effect sizes ranged from 0.53 to 0.93 , and eyes closed static CoP effect sizes ranged from 0.49 to 0.86 (see Table 3). Furthermore, the dynamic CoP time series effect size was determined to be 0.97 at 10 days post-concussion when compared to baseline measures. As such, using the lowest and more conservative effect size calculated from the literature $(0.50)$ the sample size for this study was determined. Using G*Power with a set $\alpha$ level of 0.05 , with a medium effect size (0.50) as determined from prior research findings (Cavanaugh et al., 2007; Murray et al., 2014; Powers et al., 2013; Slobounov et al., 2007), and with a desired $\beta$ of 0.20 . A total of 11 athletes per group were deemed sufficient to determine the significance at the desired power level (1- $\beta=0.80)$.

Table 3: Power analysis calculations including the means and standard deviations reported in Powers et al, and Slobounov et al, to determine the effect size needed for the proposed research sample size

\begin{tabular}{|c|c|c|c|c|}
\hline Article & Dependent Variable & $\begin{array}{l}\text { Control Group X } \\
\text { (SD) }\end{array}$ & $\begin{array}{l}\text { PC Group X } \\
\text { (SD) }\end{array}$ & $\begin{array}{l}\text { Calculated } \\
\text { ES }\end{array}$ \\
\hline \multirow[t]{7}{*}{ Powers et al } & \multirow{3}{*}{$\begin{array}{l}\text { Static Eyes Closed } \\
\text { CoP Velocities ML } \\
(\mathrm{mm}) \\
\text { CoP Velocities AP } \\
(\mathrm{mm})\end{array}$} & $\begin{array}{l}\text { Control Group } X \\
\text { (SD) }\end{array}$ & $\begin{array}{l}\text { PC Acute Group } X \\
\text { (SD) }\end{array}$ & $\begin{array}{l}\text { Calculated } \\
\text { ES }\end{array}$ \\
\hline & & $13(2)$ & $21(7)$ & 0.61 \\
\hline & & $12(2)$ & $25(5)$ & 0.86 \\
\hline & \multirow{3}{*}{$\begin{array}{l}\text { Static Eyes Closed } \\
\text { CoP Velocities ML } \\
(\mathrm{mm}) \\
\text { CoP Velocities AP } \\
(\mathrm{mm})\end{array}$} & $\begin{array}{l}\text { Control Group X } \\
\text { (SD) }\end{array}$ & $\begin{array}{l}P C R T P^{*} \text { Group } X \\
\text { (SD) }\end{array}$ & $\begin{array}{l}\text { Calculated } \\
\text { ES }\end{array}$ \\
\hline & & $13(2)$ & $19(2)$ & 0.83 \\
\hline & & $12(2)$ & $20(2)$ & 0.86 \\
\hline & Static Eyes Open & Control Group X & PC Acute Group $X^{\ominus}$ & Calculated \\
\hline
\end{tabular}


Slobounov et

CoP Velocities ML

(mm)

CoP Velocities AP

(mm)

(SD)

(SD)

ES

10 (1)

$13(3)$

0.56

$9(0.5)$

$13(0.5)$

0.97

Static Eyes Open

CoP Velocities ML

Control Group X

(SD)

$10(1)$

CoP Velocities AP

(mm)

$9(0.5)$

Baseline X

(SD)

Dynamic

CoP Time Series AP

(mm)

$0.012(0.0021)$
PC RTP* Group $X$

(SD)

Calculated

ES

$13(1)$

0.83

$13(1)$

0.93

$P C^{* *} X$

(SD)

Calculated

ES

0.97

Note: ${ }^{\ominus}=24-48$ hours post-concussion, $*=$ Return-to-play ranged from 15-48 days postconcussion, ${ }^{* *}=10$ days post-concussion. $\mathrm{SD}=$ standard deviations, $\mathrm{X}=$ mean, $\mathrm{CoP}=$ center of pressure, $\mathrm{AP}=$ anterior-posterior direction, $\mathrm{ML}=$ medial-lateral direction, $\mathrm{ES}=$ effect size. The values from Powers et al were approximated based on graphical representations of the results. 


\section{Chapter 4: RESULTS}

This cohort study examined balance performance over one week of recovery during a traditional quiet stance balance task and an environmentally relevant dynamic balance task in athletes with concussions. This research attempted to quantify the differences between an environmentally relevant dynamic balance task and a static balance task, when compared to healthy athletes, during recovery from a sport-related concussion. Data collection occurred from October 1 $1^{\text {st }}, 2013$ until April 1 ${ }^{\text {st }}, 2014$ and was analyzed using SPSS Software Package 2.0. The aims of this research were:

Aim 1: To compare recovery of postural control during a traditional quiet stance balance assessment and an environmentally relevant dynamic balance assessment, the WiiFit Soccer Heading Game, twice during one week recovery period in athletes with concussions. Aim 2: To quantify the difference between healthy individuals and athletes with concussions postural control during the administration of a quiet stance balance assessment and an environmentally relevant balance task.

Thirty one participants (Healthy Athletes $=20$; Athletes with Concussions $=11$ ) enrolled in this study and completed all assessment periods. All data analyses used a total sample size of 31. All data were considered normally distributed and as such no corrections were applied for unequal sample size. Tables 4 and 5 describe the demographics for the healthy athletes and athletes with concussions. The healthy athletes reported a total of 29 symptoms on the ImPACT Post Concussion Symptom Score (PCSS) during the second assessment period (Time 2). Furthermore, a total of 8 symptoms in the migraine cluster, 5 total symptoms in the cognitive cluster, 6 symptoms in the sleep cluster, and 10 total symptoms in the neuropsychiatric cluster 
were reported by healthy athletes on the PCSS. As reported, no healthy athletes reported more than 6 total symptoms in any of the symptom clusters on the PCSS.

Table 4: Healthy Athletes Demographics (based on $\mathrm{N}=20$ healthy athletes)

\begin{tabular}{|c|c|}
\hline & Mean (SD) \\
\hline Age (yrs) & $23.8(3.76)$ \\
\hline Weight (lbs) & $162.25(36.14)$ \\
\hline Height (in) & $67.35(3.80)$ \\
\hline Sport: & \# of Sport \\
\hline Martial Arts & 2 \\
\hline Golf & 1 \\
\hline Squash & 1 \\
\hline Soccer & 3 \\
\hline Football & 2 \\
\hline Basketball & 2 \\
\hline Volleyball & 3 \\
\hline Track \& Field & 3 \\
\hline Cross Fit & 1 \\
\hline Tennis & 1 \\
\hline Baseball & 1 \\
\hline Total & 20 \\
\hline
\end{tabular}

Note: \# = number of athletes who reported participating in that sport

Table 5: Athletes with Concussions Demographics (based on $\mathrm{N}=11$ athletes with concussions)

\begin{tabular}{|c|c|}
\hline & Mean (SD) \\
\hline Age (yrs) & 20.08 (1.44) \\
\hline Weight (lbs) & 177.41 (37.95) \\
\hline Height (in) & 71.17 (4.28) \\
\hline Sport: & \# of Sport \\
\hline Hockey & 4 \\
\hline Football & 3 \\
\hline Soccer & 1 \\
\hline Basketball & 2 \\
\hline Softball & 1 \\
\hline Total & 11 \\
\hline
\end{tabular}

Note: \# = number of athletes who reported participating in that sport. 
Athletes with concussion reported a total 168 symptoms on the PCSS during the initial assessment period (Time 1). At Time 1, a total of 92 symptoms in the migraine cluster, 46 symptoms in the cognitive cluster, 12 total symptoms in the sleep cluster, and 18 total symptoms were reported in the neuropsychiatric cluster. A total of 27 symptoms were reported on the PCSS during the second assessment period (Time 2). At Time 2, a total of 12 symptoms in the migraine cluster, 9 total symptoms in the cognitive cluster, 3 total symptoms in the sleep cluster, and 3 total symptoms were reported in the neuropsychiatric cluster. Overall, at Time 1 and Time 2 athletes with concussions reported more total symptoms on the PCSS when compared to healthy athletes. As expected, a decrease in symptoms experienced by the athletes with concussions from Time 1 to Time 2 suggests that athletes reported decreased symptoms from Time 1 to Time 2 of testing.

Across Time 1 and Time 2, no athletes with concussions or healthy athletes demonstrated a positive presence of nystagmus sign as determined by the Dix-Hallpike maneuver or the Supine Roll test. The Point-to-Point Reliability (McReynolds \& Kearns, 1983) of the Supine Roll test was determined to be 0.98 across three independent trained raters. As previously stated, The Point-to-Point Reliability of the Dix-Hallpike was not performed given the complexity of the maneuver.

\subsection{Static BaLAnCe Assessment}

In the Static A/P eyes open position, no significant main effects for time $(F(1,29)=.091$, $\mathrm{p}=.765)$ or group $(\mathrm{F}(1,29)=2.05, \mathrm{p}=.162)$ were determined (see Figure 8). Yet, in the Static A/P eyes closed position, a significant main effect of group was observed $(F(1,29)=19.065, p<$ $.001, \eta=0.988)$, yet no significant main effect of time was observed $(F(1,29)=2.39, p=.133)$ 
(see Figure 9). Follow up simple main effect of group for each time determined significant group differences at Time $1(p=.021, \eta=0.654)$ and Time $2(p<.001, \eta=.980)$. Athletes with concussions demonstrated greater Peak CoP Velocity (less stability) with eyes closed at Time 1 and Time 2 when compared to healthy individuals.

In the Static $\mathrm{M} / \mathrm{L}$ eyes open position, no significant main effects for time $(\mathrm{F}(1,29)=1.43$, $\mathrm{p}=.240)$ or group $(\mathrm{F}(1,29)=.117, \mathrm{p}=.735)$ were observed (see Figure 10). Similarly, in the Static M/L eyes closed position, no significant main effects for time $(F(1,29)=.209, \mathrm{p}=.651)$ or group $(F(1,29)=.436, \mathrm{p}=.515)$ were determined (see Figure 11$)$.

Figure 8: Peak CoP Velocity Means and Standard Deviations in the Eyes Open A/P direction during the quiet standing balance tasks.

Notes: $\mathrm{NC}=$ Healthy Athletes, and AC $=$ Athletes with Concussions, Time $1=$ the first assessment period (24-48 hours post-injury for the AC group), Time 2 = second assessment period (approximately 8 days after the first assessment period for the AC group and 7 days for the NC group). 


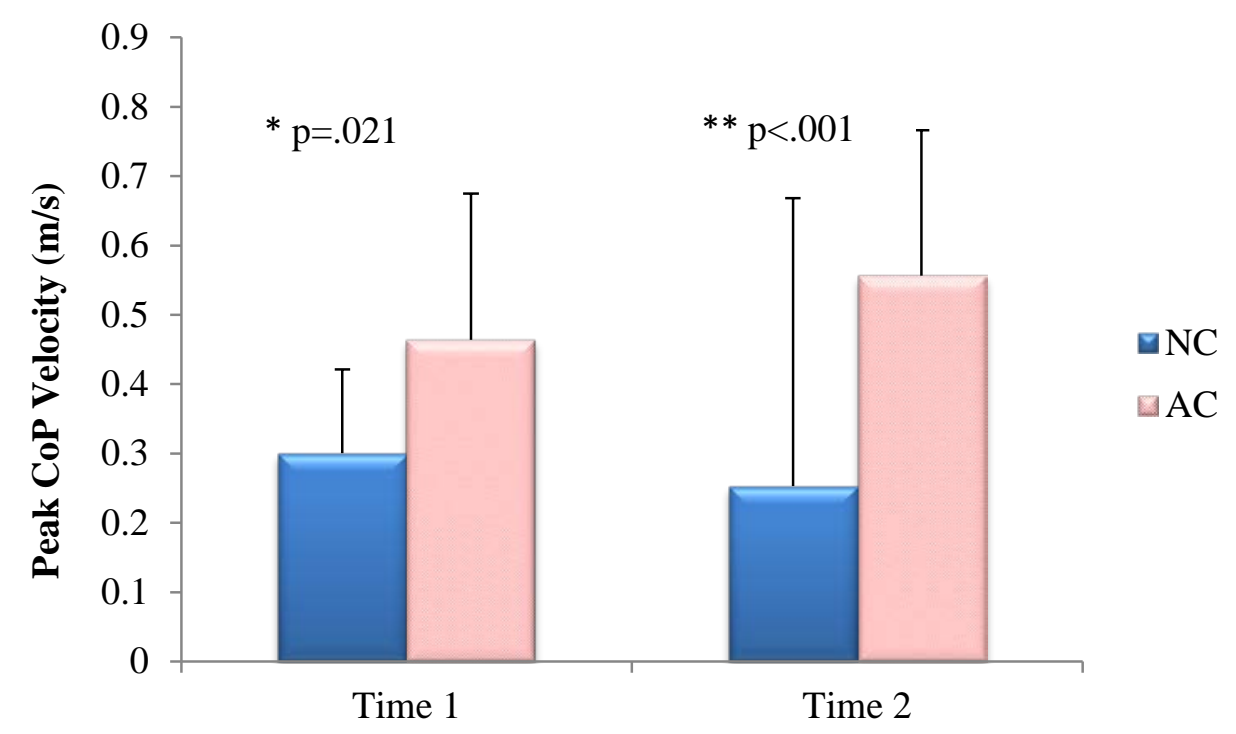

Figure 9: Peak CoP Velocity Means and Standard Deviations in the Eyes Closed A/P direction during the quiet standing balance tasks.

Notes: $\mathrm{NC}=$ Healthy Athletes, and $\mathrm{AC}=$ Athletes with Concussions, $*=$ statistical significance from between NC and AC at Time 1, ${ }^{* *}=$ statistical significance from between NC and AC at Time 2. Time 1 $=$ the first assessment period (24-48 hours post-injury for the AC group), Time 2 = second assessment period (approximately 8 days after the first assessment period for the AC group and 7 days for the $\mathrm{NC}$ group).

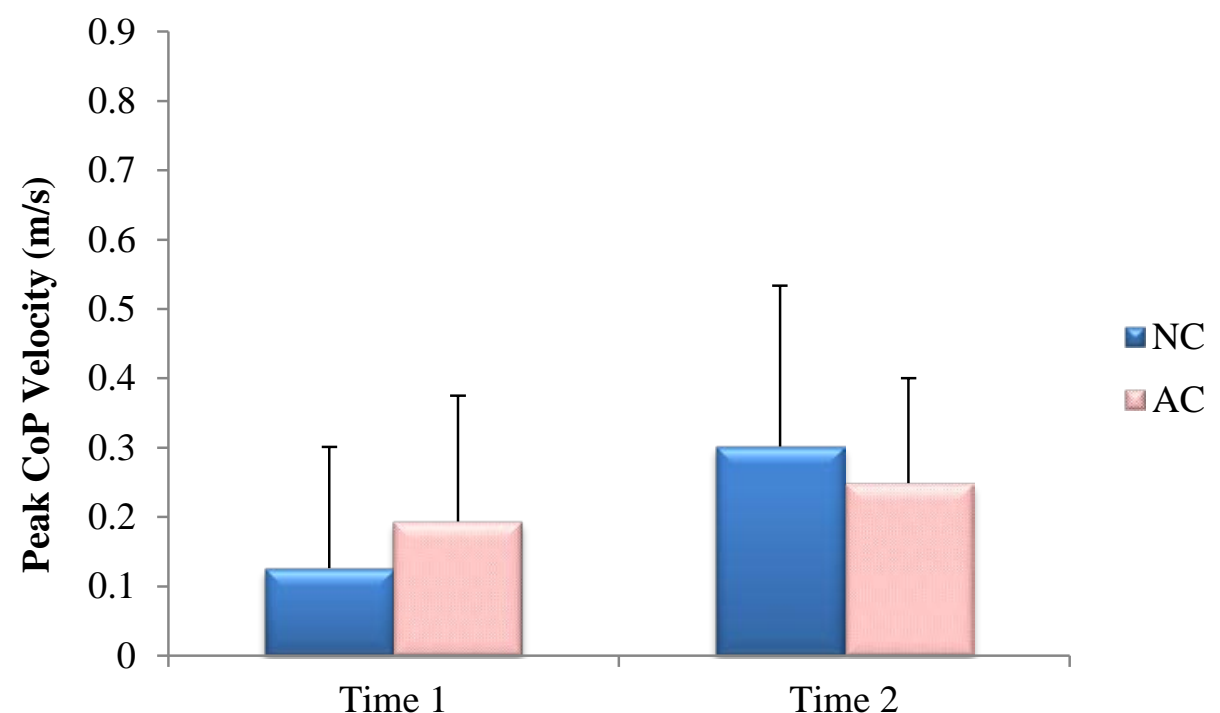

Figure 10: Peak CoP Velocity Means and Standard Deviations in the Eyes Open M/L direction during the quiet standing balance tasks.

Notes: $\mathrm{NC}=$ Healthy Athletes, and AC $=$ Athletes with Concussions. Time $1=$ the first assessment period (24-48 hours post-injury for the AC group), Time 2 = second assessment period (approximately 8 days after the first assessment period for the AC group and 7 days for the NC group). 


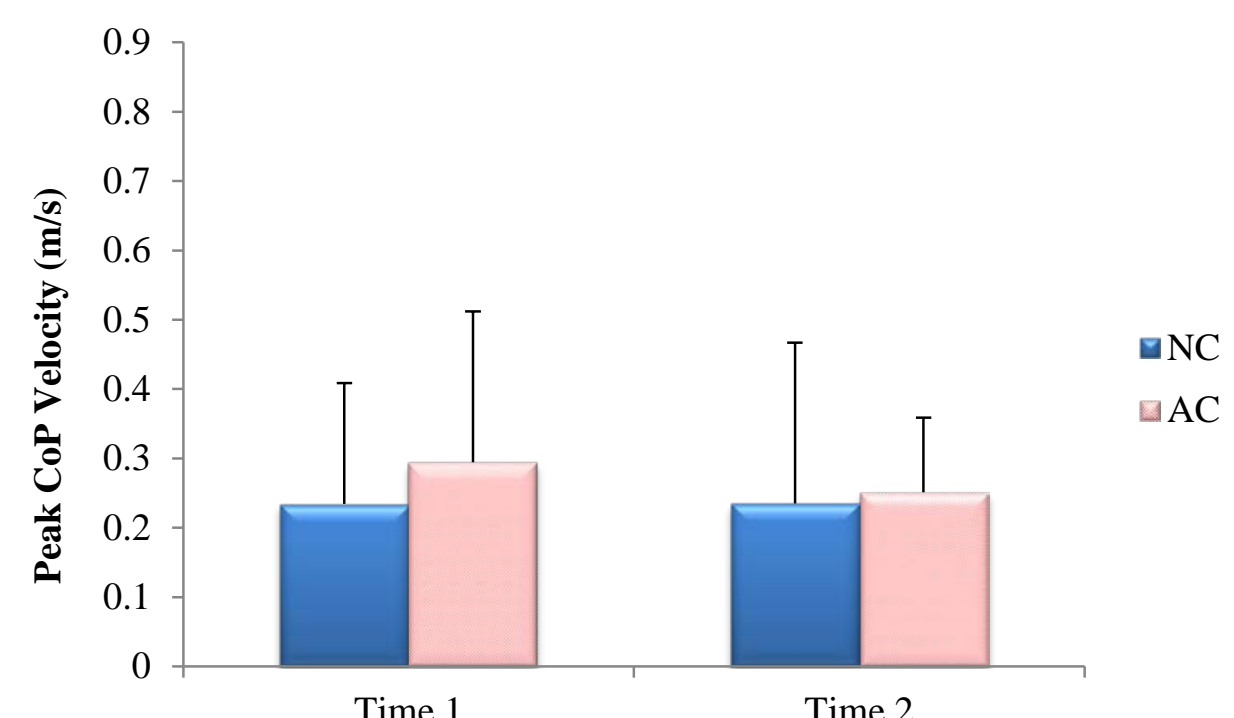

Figure 11: Peak CoP Velocity Means and Standard Deviations in the Eyes Closed M/L direction during the quiet standing balance tasks.

Notes: NC $=$ Healthy Athletes, and AC $=$ Athletes with Concussions. Time $1=$ the first assessment period (24-48 hours post-injury for the AC group), Time 2 = second assessment period (approximately 8 days after the first assessment period for the AC group and 7 days for the NC group).

\subsection{Dynamic BALANCe Assessment}

During the dynamic balance test in the A/P direction, a significant main effect of group was observed $(F(1,29)=7.20, p=.012, \eta=.737)$, however no significant main effects of time were observed $(\mathrm{F}(1,29)=.002, \mathrm{p}=.963)$ (see Figure 12). Follow up simple main effect of group for each time determined a significant difference at Time $1(\mathrm{p}=.013, \eta=.722)$ and Time $2(\mathrm{p}=$ $.041, \eta=.543)$

In the $\mathrm{M} / \mathrm{L}$ direction during the dynamic balance test, a significant main effect of group was determined $(\mathrm{F}(1,29)=10.58, \mathrm{p}=.003, \eta=.882)$, and a significant main effect of time was determined $(F(1,29)=6.28, p=.018, \eta=.678)$ (see Figure 13). Follow up simple main effects of group for each time determined significant group differences at Time $1(p=.005, \eta=0.840)$ and Time $2(p=.029, \eta=.605)$. In addition, follow up main effects of time for each group 
determined no significant group differences in Athletes with Concussion $(\mathrm{p}=.093)$ and the healthy athletes $(\mathrm{p}=.412)$. As such, athletes with concussions had significantly greater Peak CoP Velocity (less stability) in both the A/P and the M/L directions at Time 1 and Time 2 when compared to healthy individuals.

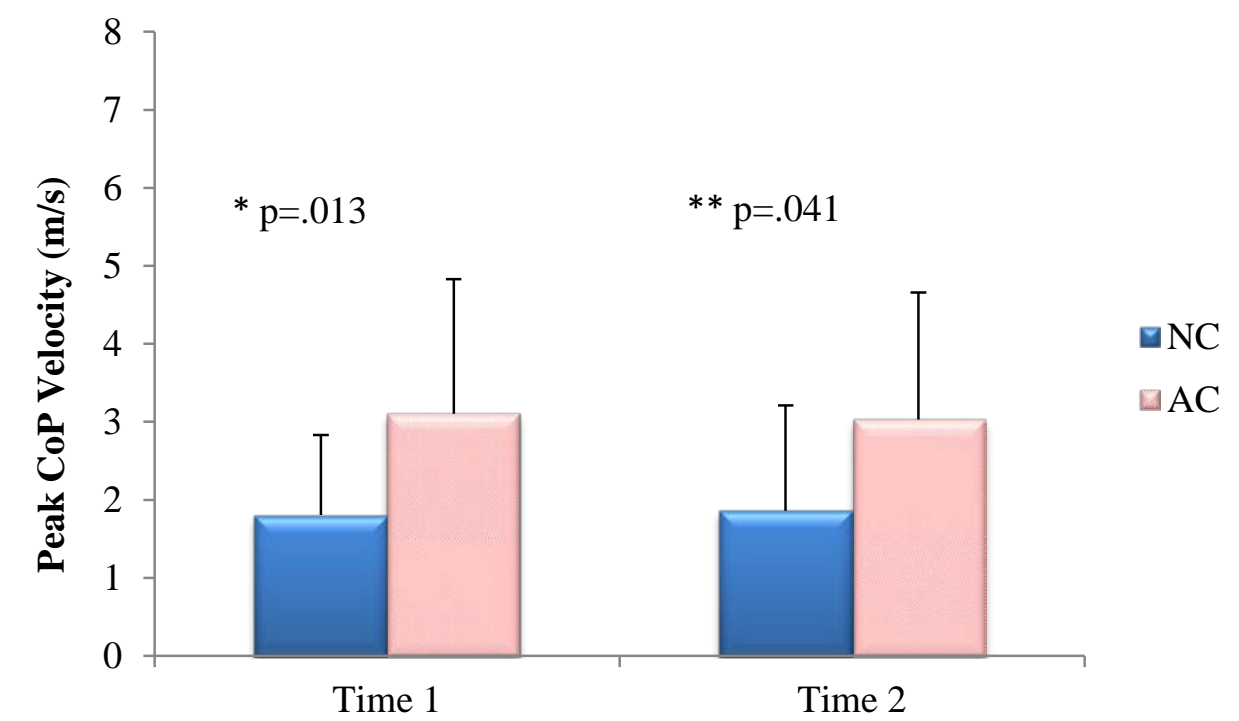

Figure 12: Peak CoP Velocity Means and Standard Deviations in the A/P direction during the WiiFit Soccer Heading Game.

Notes: $\mathrm{NC}=$ Healthy Athletes, and AC $=$ Athletes with Concussions, $*=$ statistical significance from between NC and AC at Time 1, ** = statistical significance from between NC and AC at Time 2. Time 1 $=$ the first assessment period (24-48 hours post-injury for the AC group), Time $2=$ second assessment period (approximately 8 days after the first assessment period for the AC group and 7 days for the NC group). 


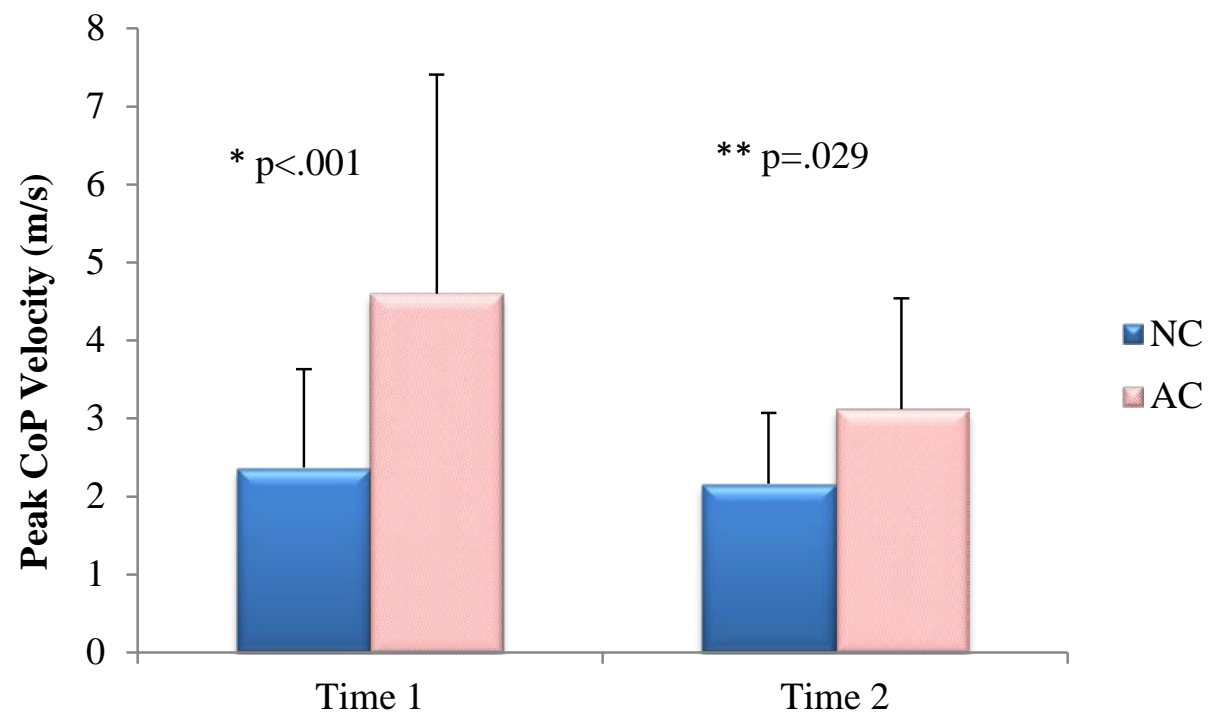

Figure 13: Peak CoP Velocity Means and Standard Deviations in the M/L direction during the WiiFit Soccer Heading Game.

Notes: $\mathrm{NC}=$ Healthy Athletes, and AC $=$ Athletes with Concussions, $*=$ statistical significance from between NC and AC at Time $1, * *=$ statistical significance from between NC and AC at Time 2. Time 1 $=$ the first assessment period (24-48 hours post-injury for the AC group), Time $2=$ second assessment period (approximately 8 days after the first assessment period for the AC group and 7 days for the NC group). 


\section{Chapter 5: DISCUSSION}

\subsection{OVERVIEW}

The purpose of this study is to compare and contrast changes in postural control during a traditional quiet stance balance assessment and an environmentally relevant dynamic balance assessment during one week of recovery in athletes with concussions compared to healthy controls, and to track performance on these two assessments post-concussion to determine their relationship and sensitivity to the recovery of a concussion. The aims of this research were (1) to compare recovery of postural control during a traditional quiet stance balance assessment and an environmentally relevant dynamic balance assessment, the WiiFit Soccer Heading Game, twice during a one week recovery period in athletes with concussions; and (2) to quantify the difference between healthy individuals and athletes with concussions postural control during the administration of a quiet stance balance assessment and an environmentally relevant balance task. It was hypothesized that (1) athletes with concussions would recover static balance within one week post-injury whereas the dynamic balance assessment balance impairment would persist beyond one week when compared to healthy athletes; and (2) the dynamic balance assessment would be a more challenging motor control task, as demonstrated by greater postural instability in multiple planes of motion, when compared to the static balance assessment.

The hypotheses of this study were partially met. During the static and dynamic balance assessments at Time 1 and Time 2 athletes with concussions demonstrated significantly less stability when compared to healthy athletes. Moreover, the control of balance in athletes with concussions did not recover within the prior specified and proposed timeline as determined by either static or dynamic test. Prior research using static tests of balance, such as the BESS and the SOT, reported recovery of postural stability within 72 hours (3 days) post-injury (Bell et al., 
2011; Guskiewicz et al., 2001; McCrea et al., 2003; Riemann \& Guskiewicz, 2000). The results of this study do not support these findings. The results indicate that the evaluation of static balance through traditional measurements, such as the BESS and the SOT, may be less sensitive in detecting postural control deficits after a concussion injury. Powers and colleagues (2013) determined that through the use of force platforms, CoP RMS velocity, in the eyes' closed conditions did not return to normal at the time of 15-48 days post-injury. Powers et al. (2013) findings and the current study's findings support the use of dynamic measurements and the use of CoP over time to track balance dysfunction in athletes with concussions.

\subsection{Static Balance Assessment}

The results of this study show that athletes with concussions were less stable in the A/P direction with the eyes closed during the static balance assessment at eight days post-concussion when compared to healthy athletes. Furthermore, from Time 1 to Time 2 athletes with concussions demonstrated poorer stability in the eyes closed A/P condition. In contrast, no significant differences were observed between the Peak CoP Velocity within the athletes with concussions group over the two assessment periods.

One explanation is that, during the static balance task, the base of support is reduced as the participants stand with their ankles touching (medial malleolus touching medial malleolus of each ankle). This unstable position forces most individuals to adopt a feed-back reflexive strategy controlled by the ankle extensors in order to maintain an upright stance (Powers et al., 2013; Winter, 1995). The feed-back model of postural control relies heavily on a succinct coordination between all three sensory systems (Winter, 1995). The reduction of visual sensory information during the eyes closed condition increases the reliance upon the vestibular and 
somatosensory systems to interpret environmental afferent cues. In the absence of visual information, the vestibular system is the main source of spatial orientation information (bodies location in space) to prevent an unexpected fall. During the assessment, somatosensory information was available to participants along with higher weighted vestibular information. It can be postulated that inaccurate sensory information was applied to the postural control system as a result of potential tissue damage. This tissue damage could result in an inflammatory response of the damaged and/or subsequent supporting tissue which could delay or impair neurotransmitter function. The vestibular system damage could be either physical damage to the vestibular organs, or the vestibulospinal tract, or the vestibular nuclei, or a downstream effect of tissue inflammation, or a combination of all four.

The potential contribution of vestibular system damage is also supported by the lack of differences in the eyes open static balance assessment condition between groups at either Time 1 and Time 2. In the eyes open condition, the eyes are the most heavily weighted source of afferent spatial information (Horak, Nashner, \& Diener, 1990). During the assessment, the participants were instructed to gaze into a solid white projection screen with medium room lighting (Figure 6). The goal of the task was to maintain the static upright position and to minimize movement. The field of view of the participants was not occluded with the white projector screen in order to facilitate the use of both central and peripheral visual cues to maintain upright stance. However, any distractors, such as investigators and the control room, were removed from the field of view in order to mitigate participant errors. In the presence of visual afferent information, the maintenance of upright stance became easier, and participants were more stable as evidenced by lower Peak CoP A/P Velocity. Potentially peripheral and central visual field cues provided enough information that made the task easier to accomplish when compared to the absence of 
visual sensory information in the eyes closed condition. In short, the static quiet stance balance assessment is an easier task and posture is more stable when the eyes are open. Conversely, with the eyes closed maintaining an upright stance becomes more difficult due in part to the increased reliance upon the vestibular and somatosensory systems and the general decrease in the amount of spatial information available to the participant. The lack of recovery of postural control, as evidence by greater Peak CoP A/P velocity, in the eyes closed conditions in the athletes in the concussion group indicates that damage to the vestibular system is one, but not the only, viable explanation of these results

The observed postural instability in athletes with concussions during recovery supports the notion of potential damage to the vestibular system (Guskiewicz, 2011; McCrea et al., 2003; Powers et al., 2013). Multiple studies have provided similar conclusions using subjective and objective measures of balance (Guskiewicz et al., 2001; Guskiewicz, 2011; McCrea et al., 2003; Powers et al., 2013; Riemann \& Guskiewicz, 2000). Powers et al. (2013) noted increased CoP velocity in athletes with concussions, when compared to baseline measures, which persisted beyond ten days of recovery. They concluded that the persisting postural instability could be related to a vestibular system dysfunction, particularly the vestibulospinal tract. The ankle extensors receive direct input from the descending lateral vestibulospinal tract (Highstein \& Holstein, 2012). If impairment were present in the descending lateral vestibulospinal tract, feedback postural control of the ankle extensors would be inaccurate and thus result in greater postural instability. Our results may also support this explanation as we observed postural instability at both testing times in the eyes closed condition in the A/P direction.

Further studies that examined postural stability in individuals with acute and chronic unilateral peripheral vestibular dysfunction (UPVD) reported results similar to the current study 
(Fujimoto et al., 2012; Fujimoto et al., 2014). Fujimoto et al. (2012) noted that individuals with UPVD had greater CoP velocity in the eyes closed condition in the A/P direction when compared to healthy controls. Further research by Fujimoto et al. (2014) observed similar findings in individuals with vestibular neuritis. The postural instability noted across similar measurement techniques and two different types of injuries, concussion and UPVD, demonstrate potential relationships between the injuries. Thus, the speculation arises that damage to the vestibular system, either direct or indirect, could result in the observed postural instability in athletes with concussions of this study. However, direct damage to the vestibular system is highly suspect in the current study because the no nystagmus was observed in the athletes with concussions during eight days of recovery. Again, nystagmus is uncontrolled or un-purposeful eye movement(s) that is directly linked to vestibular organ damage (Isaradisaikul et al., 2010). The lack of BBPV, as observed by a negative Dix-Hallpike maneuver and Supine Roll test, indicates that no otoliths were present in the vestibular organs at the time of testing. As such, vestibular organ damage was unlikely to have occurred from the concussion injury in this studies sample.

Two main theories may offer potential explanations for these abnormal postural control results: (1) Damage to the vestibulospinal tract or (2) damage to the vestibular nuclei itself or interference to the vestibular nuclei pathways. Damage to the vestibulospinal tract is plausible, but without direct measurements this speculation cannot be confirmed by the current research. Furthermore, the vestibulospinal tract receives information for motor commands that modulate muscular responses from the vestibular nuclei (Kandel, Schwartz, \& Jessell, 2000). The results indicate greater instability in the replication of quiet stance in athletes with concussions. The variable, Peak CoP Velocity, is a second order variable that approximates the control mechanisms of posture at the higher order sensory integration level (Nashner et al., 1982; Powers 
et al., 2013; Winter, 1995). It can be assumed that higher order cortical influences are interrupted as a result of a concussion injury rather than descending motor pathways such as the vestibulospinal tract. This higher order cortical interruption could be expressed as damage to the vestibular nuclei or vestibular nuclei pathways. This in turn would result in inaccurate sensory feed-back information applied to the thalamus. If an inappropriate signaling is applied to the thalamus, abnormal postural control is expected. However, without the use of brain imaging or direct measurements of the brain, this observation is speculative. Further research is needed to extrapolate where the proposed vestibular contributions to posture are disturbed in athletes with concussions. Lastly, research is needed to ascertain if and when static postural control returns to normal healthy levels.

\subsection{Dynamic BaLAnCe Assessment}

The results of the dynamic balance task observed greater instability in the A/P and M/L directions during recovery in athletes with concussions. During the WiiFit Soccer Heading Game participants must move their body in mediolateral directions in order to move the on screen avatar to interact with the soccer balls and dodge the distraction objects. Although the participants are in a stable base of support (feet spread shoulder width apart) with visual, vestibular, and somatosensory information present, greater instability was still observed in both planes of body movement. This finding is not surprising given that the WiiFit Soccer Heading Game replicates game-like demands and functional abilities more efficiently when compared to the static balance assessment.

As prior evidence has suggested, damage from a concussion injury is functional rather than structural (Lovell, 2009; Shaw, 2002). The functional deficits are expressed as a 
neurometabolic cascade that could affect feed-back or feed-forward signals to the areas of the brain responsible for postural control. During a challenging task in a rapidly changing environment, the CNS can weigh incoming sensory information and apply muscular contractions in order to regulate postural control (Guskiewicz, 2011; Klous et al., 2011; Shumway-Cook \& Horak, 1986). As the environmental context increases, the stress levels upon the visual, vestibular, and somatosensory systems increase as well (Horak, 1987). This has been demonstrated in multiple research studies that have reported lingering balance dysfunction beyond the traditional 72 hour mark (Catena et al., 2009; Parker et al., 2007; Slobounov et al., 2006; Slobounov et al., 2007; Slobounov et al., 2008). Slobounov and colleagues (2006) presented a moving visual field to athletes with concussions, where the front and back of a computer generated moving wall altered its spatial position. In the presence of the virtual front moving and roll moving room, the difference in $\mathrm{CoP}$ displacement was observed in athletes with concussions when compared to baseline measures and assessment at 10 and 30 days postconcussion. The current results support previously published findings that athletes with concussions are more unstable beyond the initial date of injury in virtual moving unanticipated dynamic balance assessment. Virtual environments place the athlete in an environmentally relevant context that increases the functional demands on all of the sensory systems. Thus, greater postural instability in multiple planes of body motion is expected.

Slobounov and colleagues (2007) observed similar results to the early work conducted in 2006. Athletes with concussions demonstrated greater instability until day 17 of recovery, as measured on the SOT, in an environmentally relevant balance task. However, a major limitation of this work and Slobounov's early work is that participants were instructed to maintain quiet stance during evaluation. The current study expanded upon previous research and provided not 
only an environmentally relevant context, but a goal oriented task which required athletes to interact with objects within the environment. Given that the aforementioned studies only reported significant results in the A/P CoP directions, it is speculated that the WiiFit Soccer Heading Game is a more sensitive tool to uncover postural control deficits. It could be that greater instability is revealed when the participant must direct their posture using feed-forward control. Feed-forward control of posture is hypothesized to be driven and regulated by higher order neural controllers (Klous et al., 2011). This unknown higher order neural control mechanism relies heavily on available sensory information and proper sensory integration. If either the flow or integration of sensory information is interrupted, abnormal postural control responses should be expected. Furthermore, the WiiFit Soccer Heading Game more accurately simulates game-like scenarios and behaviors when compared to the static balance assessment. The simple movement into multiple planes of motion as per game demands more accurately describes true human motion. Thus, the greater instability in the $\mathrm{A} / \mathrm{P}$ and $\mathrm{M} / \mathrm{L}$ is inherently expected.

A recent study by Murray and colleagues (2014) used the WiiFit Soccer Heading Game as an environmentally relevant balance task to measure, with a monocular eye tracker, the eye movements of athletes with concussions within 24-48 hours post-injury. They determined that an interruption in the vestibular-ocular reflex was present within 48 hours of recovery. Furthermore, the WiiFit Soccer Heading Game score was not significantly different over two weeks of recovery, whereas healthy individuals significantly increased. Without force platform data, the results of this study are limited. However, it can be concluded from this data that in the presence of an environmentally relevant balance task, specifically the WiiFit Soccer Heading Game, residual balance dysfunction is present at two weeks of post-concussion. The present study 
expanded on these findings by adding force platform data. As previously stated, a significant difference in the Peak CoP Velocity in $\mathrm{A} / \mathrm{P}$ and $\mathrm{M} / \mathrm{L}$ directions during recovery was observed. The previous study, along with the current study results, support the notion that environmentally relevant balance tasks are more sensitive to postural control instabilities over time when compared to traditional static balance tasks. The increased environmental context with goal specific tasks could be interpreted as a dual task. As such, examining athletes with concussions under dual task paradigms is warranted.

Previously published studies that involved dynamic motor performance tasks, such as walking (gait) coupled with a concurrent cognitive load or dual task, demonstrated residual motor dysfunctions that remained until four weeks post-concussion recovery (Catena et al., 2009; Parker, Osternig, Lee, Donkelaar, \& Chou, 2005; Parker et al., 2007). The results of the current study cannot be compared to gait analysis due to the inherent differences in evaluation techniques; however, similarities can be drawn. Parker and colleagues (2007) examined gait characteristics in athletes with concussions and matched controls (COM velocity, COM-CoP separation distance, and COM sway) while participants performed a dual task. It was concluded that athletes with concussions had residual motor dysfunction as presented by slower COM velocity, smaller COM-CoP separation distance, and greater COM sway.

Similar results were reported by Catena and colleagues (2009). Catena et al. followed analogous methods as reported in Parker et al. (2005), including the same dual task. The reported findings suggested that, during the dual task gait performance, athletes with concussions were significantly worse than matched controls until day 6 of recovery. Findings by Catena et al. (200), Parker et al. (2007) and Murray et al. (2014) suggest a dynamic movement reduction in 
motor control function in athletes with concussions. Moreover, this reduction in motor control could be primarily due to the increased cognitive load imposed by the dual task.

Although the present study did not measure or report cognition, it can be postulated that the WiiFit Soccer Heading Game introduces a significant increase in sensory demands and cognitive load when compared to the quiet standing task. The cognitive load or dual task possibilities are reflected in the requirement that athletes must intentionally direct their on screen avatar to interact with the soccer balls and dodge distraction objects. This creates an increased neurological load on the brain that has already experienced damage. As a results of this increased cognitive load we see reduced postural stability in multiple planes of motion (A/P and M/L) rather than the observed single plane motion deficits (A/P only) measured during quiet stance. Perhaps the use of both feed-back and feed-forward mechanisms to maintain an upright stance resulted in decreased postural stability, in athletes with concussions.

As previously stated, feed-forward and feed-back signals are critical for preparing and directing muscular responses in an unexpected environment. The WiiFit Soccer Heading Game is a true unexpected environment. It randomizes the objects presented to the player in terms of speed of delivery and differing objects. This randomization provides an irregular and unexpected environment during each round of play. As a direct result, the WiiFit Soccer Heading Game more accurately replicates game-like scenarios. The athletes then must properly integrate the available sensory information and disseminate it accordingly. If improper integration and weighting of sensory system cues occurs, than an inaccurate postural response is performed. The results of the current study determined that athletes with concussions were more unstable during the WiiFit Soccer Heading Game. Therefore, it can be suggested that improper integration or 
weighting of sensory cues are present when athletes with concussions play the WiiFit Soccer Heading Game.

The basal ganglia and its projections are the postulated structure responsible for sensory integration or weighting of sensory cues (Alexander, DeLong, \& Strick, 1986). However, it should be noted that any upstream or downstream mechanisms that are interrupted from a concussion injury could influence basal ganglia function. As such, multiple alternative brain structures or pathways could be the point of interruption in the afferent or efferent flow of information in the brain leading to the basal ganglia after a concussion injury. Thus, the basal ganglia can only be considered one of the many candidate mechanisms responsible for the postural instability observed in this study.

The basal ganglia are a higher order structure that projects into and passes through many cortical circuits and structures (Knierim, 2012). The direct pathway of the basal ganglia further excites the motor cortex, while the indirect pathway inhibits the cortex. Both pathways use similar structures to deliver the appropriate positive or negative feed-back to the cortex. In addition, the basal ganglia circuits involve motor and non-motor regions that extend to multiple superficial cerebral cortex areas (Alexander et al., 1986). During a concussion injury, it is unlikely that direct damage to the basal ganglia will occur. However, it is plausible that basal ganglia-thalamocortical circuits could be interrupted due to the skull and a superficial cerebral interaction. The interruption will influence basal ganglia function. Incorrect function is exhibited by a variation of concussion symptoms, but more importantly abnormal balance or postural control. This observation is further supported by abnormality in certain dynamic tasks, such as Gait Initiation and Gait Termination, in athletes with concussions (Buckley, Munkasy, TapiaLovler, \& Wikstrom, 2013; Buckley, 2014). Similar abnormal Gait Initiation and Gait 
Termination have also been observed in individuals with Parkinson's disease, where the primary brain structure responsible for the abnormal movement is postulated to be the basal ganglia (Cameron, Murphy, Morris, Raghav, \& Iansek, 2010; Fernandez et al., 2013; Sparrow \& Tirosh, 2005). However, this suggestion is highly speculative given the vast differences between Parkinson's disease and a concussion injury. In addition, comparisons between Parkinson’s disease and a concussion injury are beyond the scope of this article. Further research is needed to substantiate this unlikely relationship.

\subsection{CLINICAL IMPLICATIONS}

Given the findings reported in this study, one can postulate that as the environmental and cognitive load increases, a greater disparity in the biomechanical measures emerges in athletes with concussions. Surprisingly, these results indicate that athletes with concussions could be experiencing postural instability far beyond the proposed RTP criteria when evaluated in a gamelike scenario. To date, no known assessments of balance are currently used to monitor concussion recovery within a game-like scenario. The lack of sensitive balance assessments could lead to releasing an athlete too soon and an increased likelihood of the athlete experiencing a second concussion when their brain is not fully recovered. Moreover, this could result in the athlete incurring Second Impact Syndrome that could advance to lifelong neurological impairments or even death (Cantu, 1998). Thus, quiet standing balance tasks only expose one facet of balance impairments in athletes with concussions; whereas during an environmentally relevant balance task, balance impairments could be presented in other previously unexplored details. The coupling of a static balance task and an environmentally relevant dynamic balance task may provide a more robust diagnosis of a concussion injury and better monitor of 
concussion recovery. Lastly, the lack of a significant finding in the static quiet standing balance assessment eyes open condition suggest that simple static quiet standing balance tasks could be ineffective methods of measuring balance dysfunction post-concussion.

\subsection{STUDY LIMITATIONS}

This study had several limitations. The limitations included data collection methods, sample population, and instrumentation. These limitations could limit the generalizability and accuracy of the study.

The data collection methods of this study included a novel assessment of balance. The WiiFit has considerable support for its reliability and validity for use in clinical populations. In addition, this study used a force platform for the assessment of posture. A force platform is considered the gold standard to assess posture. However, the WiiFit Soccer Heading Game does not currently have any reliability or validity data to support its use in concussion populations. This presents a threat to the internal validity of the study.

The sample included a convenience sample that was drawn from the surrounding participating CMC physicians, athletic trainers, and athletes. The athletes who participated in this study were from a variety of sports and sample did not include matched healthy controls. When dealing with clinical populations, it is near to impossible to have a random sample population. Nonetheless, the present sample of this research presents a threat to the external validity of the study.

Lastly, the instrumentation used in this study presents a concern for the internal validity of the current research. Force platforms are considered the gold standard by which posture is evaluated. However, placing the WiiFit balance board on top of the force platform has not been 
previously done in other studies. The additionally raised platform of the WiiFit balance board could have influenced the results of the study by slightly reducing the accuracy of the data. However, all participants were assessed during all balance tasks with the WiiFit balance board placed on top of the force platforms. As such, the methods are replicable but the reduction in the accuracy of the data is a threat to the internal validity of the study.

\subsection{FUTURE RESEARCH CONSIDERATIONS}

Further investigation beyond the current study’s limited recovery period is warranted. If dynamic balance tasks are more sensitive in detecting balance impairments when compared to static balance tasks, it is of interest to determine if and when static and dynamic balance recovers. Given the presented data and prior research; it is warranted to examine the timeline of recovery beyond thirty days post-injury. In addition, comparisons between current clinical balance assessments, such as the BESS, the SOT, and the Romberg Test, and an environmentally relevant balance task are warranted. Expanded research on this topic should include exploratory evaluations of any relationship(s) to recovery as denoted on the ImPACT test. Moreover, comparisons between cognitive recovery and balance recovery (acutely and longitudinally) are of particular interest.

\subsection{SUMMARY AND CONCLUSION}

The current study investigated changes in postural control during a traditional quiet stance balance assessment and an environmentally relevant dynamic balance assessment, the WiiFit Soccer Heading Game, during one week of recovery in athletes with concussions. From the results of the study, an environmentally relevant dynamic balance task was more challenging 
and is also a more sensitive balance assessment when compared to the traditional quiet stance balance assessment. Our study revealed that both quiet standing and dynamic balance assessments show lingering balance dysfunction after approximately eight days post-concussion. In addition, this study supports the novel use of an environmentally relevant dynamic balance assessment, the WiiFit Soccer Heading Game, to evaluate a concussion and to monitor the recovery of a concussion. Potentially coupling quiet standing and an environmentally relevant dynamic balance assessment could aid clinicians in making more reliable diagnostic and recovery recommendations. Moreover, it is the opinion of the author that the lingering balance dysfunction is related to persisting higher order cortical damage or sensory integration issues. However, without imaging to support this claim, this postulation is speculative at best. Further research is needed to expand these results to include the entire recovery period from an athletic concussion. 


\section{REFERENCES}

Alexander, G. E., DeLong, M. R., \& Strick, P. L. (1986). Parallel organization of functionally segregated circuits linking basal ganglia and cortex. Annual Review of Neuroscience, 9, 357381.

Aubry, M., Cantu, R., Dvorak, J., Graf-Baumann, T., Johnston, K., Kelly, J., . . Concussion in Sport Group. (2002). Summary and agreement statement of the first international conference on concussion in sport, Vienna 2001. Recommendations for the improvement of safety and health of athletes who may suffer concussive injuries. British Journal of Sports Medicine, 36(1), 6-10.

Bell, D. R., Guskiewicz, K. M., Clark, M. A., \& Padua, D. A. (2011). Systematic review of the balance error scoring system. Sports Health, 3(3), 287-295. doi:10.1177/1941738111403122

Blennow, K., Hardy, J., \& Zetterberg, H. (2012). The neuropathology and neurobiology of traumatic brain injury. Neuron, 76(5), 886-899. doi:10.1016/j.neuron.2012.11.021; 10.1016/j.neuron.2012.11.021

Buckley, T. A. (2014). Acute and lingering impairments in post-concussion postural control. In S. M. Slobounov, \& W. J. Sebastianelli (Eds.), Concussions in athletics (pp. 139-165). New York, New York: Springer New York. doi:10.1007/978-1-4939-0295-8_8

Buckley, T. A., Munkasy, B. A., Tapia-Lovler, T. G., \& Wikstrom, E. A. (2013). Altered gait termination strategies following a concussion. Gait \& Posture, 38(3), 549-551. doi:10.1016/j.gaitpost.2013.02.008 [doi]

Cameron, D., Murphy, A., Morris, M. E., Raghav, S., \& Iansek, R. (2010). Planned stopping in people with Parkinson. Parkinsonism \& Related Disorders, 16(3), 191-196. doi:10.1016/j.parkreldis.2009.11.008 [doi]

Cantu, R. C. (1998). Second-impact syndrome. Clinics in Sports Medicine, 17(1), 37-44.

Catena, R. D., van Donkelaar, P., \& Chou, L. S. (2009). Different gait tasks distinguish immediate vs. long-term effects of concussion on balance control. Journal of NeuroEngineering and Rehabilitation, 6, 25-0003-6-25. doi:10.1186/1743-0003-6-25; 10.1186/1743-0003-6-25

Cavanaugh, J. T., Mercer, V. S., \& Stergiou, N. (2007). Approximate entropy detects the effect of a secondary cognitive task on postural control in healthy young adults: A methodological report. Journal of NeuroEngineering and Rehabilitation, 4(42), 1-7.

Cavanaugh, J. T., Guskiewicz, K. M., \& Stergiou, N. (2005). A nonlinear dynamic approach for evaluating postural control: New directions for the management of sport-related cerebral concussion. Sports Medicine (Auckland, N.Z.), 35(11), 935-950. 
Cavanaugh, J. T., Guskiewicz, K. M., Giuliani, C., Marshall, S., Mercer, V. S., \& Stergiou, N. (2006). Recovery of postural control after cerebral concussion: New insights using approximate entropy. Journal of Athletic Training, 41(3), 305-313. Retrieved from

Center for Disease Control and Prevention. Heads ups: Facts for physicians about mild traumatic brain injury. ( No. CS109152).U.S. Department of Health and Human Services.

Centers for Disease Control and Prevention (CDC). (2006). Incidence rates of hospitalization related to traumatic brain injury--12 states, 2002. Morbidity and Mortality Weekly Report, 55(8), 201-204.

Clark, R. A., Bryant, A. L., Pua, Y., McCrory, P., Bennell, K., \& Hunt, M. (2010). Validity and reliability of the Nintendo Wii balance board for assessment of standing balance. Gait \& Posture, 31(3), 307-310. doi:10.1016/j.gaitpost.2009.11.012

Covassin, T., Elbin III, J., Stiller-Ostrowski, J. L., \& Kontos, A. P. (2009). Immediate postconcussion assessment and cognitive test (ImPACT) practices of sports medicine professionals. Journal of Athletic Training, 44(6), 639-644.

Fernandez, K. M., Roemmich, R. T., Stegemoller, E. L., Amano, S., Thompson, A., Okun, M. S., \& Hass, C. J. (2013). Gait initiation impairments in both essential tremor and Parkinson's disease. Gait \& Posture, 38(4), 956-961. doi:10.1016/j.gaitpost.2013.05.001 [doi]

Fife, T. D., Iverson, D. J., Lempert, T., Furman, J. M., Baloh, R. W., Tusa, R. J., . . Quality Standards Subcommittee, American Academy of Neurology. (2008). Practice parameter: Therapies for benign paroxysmal positional vertigo (an evidence-based review): Report of the quality standards subcommittee of the American academy of neurology. Neurology, 70(22), 2067-2074. doi:10.1212/01.wnl.0000313378.77444.ac [doi]

Fujimoto, C., Egami, N., Kinoshita, M., Sugasawa, K., Yamasoba, T., \& Iwasaki, S. (2014). Postural stability in vestibular neuritis: Age, disease duration, and residual vestibular function. The Laryngoscope, 124(4), 974-979. doi:10.1002/lary.24342 [doi]

Fujimoto, C., Murofushi, T., Sugasawa, K., Chihara, Y., Ushio, M., Yamasoba, T., \& Iwasaki, S. (2012). Assessment of postural stability using foam posturography at the chronic stage after acute unilateral peripheral vestibular dysfunction. Otology \& Neurotology, 33(3), 432-436. doi:10.1097/MAO.0b013e3182487f48 [doi]

Garrick, J. G. (2005). Unreported concussion in high school football players. Clinical Journal of Sport Medicine : Official Journal of the Canadian Academy of Sport Medicine, 15(5), 385; author reply 385.

Gessel, L. M., Fields, S. K., Collins, C. L., Dick, R. W., \& Comstock, R. D. (2007). Concussions among united states high school and collegiate athletes. Journal of Athletic Training, 42(4), 495-503. 
Guskiewicz, K. M. (2011). Balance assessment in the management of sport-related concussion. Clinics in Sports Medicine, 30(1), 89-102, ix. doi:10.1016/j.csm.2010.09.004

Guskiewicz, K. M., Mihalik, J. P., Shankar, V., Marshall, S. W., Crowell, D. H., Oliaro, S. M., . . . Hooker, D. N. (2007). Measurement of head impacts in collegiate football players: Relationship between head impact biomechanics and acute clinical outcome after concussion. Neurosurgery, 61(6), 1244-52; discussion 1252-3. doi:10.1227/01.neu.0000306103.68635.1a

Guskiewicz, K. M., Ross, S. E., \& Marshall, S. W. (2001). Postural stability and neuropsychological deficits after concussion in collegiate athletes. Journal of Athletic Training, 36(3), 263-273.

Harmon, K. G., Drezner, J. A., Gammons, M., Guskiewicz, K. M., Halstead, M., Herring, S. A., . . Roberts, W. O. (2013). American medical society for sports medicine position statement: Concussion in sport. British Journal of Sports Medicine, 47(1), 15-26. doi:10.1136/bjsports2012-091941; 10.1136/bjsports-2012-091941

Highstein, S. M., \& Holstein, G. R. (2012). The anatomical and physiological framework for vestibular prostheses. Anatomical Record, 295(11), 2000-2009. doi:10.1002/ar.22582; 10.1002/ar.22582

Holmes, J. D., Jenkins, M. E., Johnson, A. M., Hunt, M. A., \& Clark, R. A. (2012). Validity of the Nintendo Wii(R) balance board for the assessment of standing balance in Parkinson's disease. Clinical Rehabilitation, 27(4) doi:10.1177/0269215512458684

Horak, F. B. (1987). Clinical measurement of postural control in adults. Physical Therapy, 67(12), 1881-1885.

Horak, F. B., Nashner, L. M., \& Diener, H. C. (1990). Postural strategies associated with somatosensory and vestibular loss. Experimental Brain Research. 82(1), 167-177.

Howley, E. T., \& Thompson, D. L. (2012). Fitness professional's handbook (6th ed.). Champaign, IL: Human Kinetics.

Ingersoll, C. D., \& Armstrong, C. W. (1992). The effects of closed-head injury on postural sway. Medicine and Science in Sports and Exercise, 24(7), 739-743.

Isaradisaikul, S., Navacharoen, N., Hanprasertpong, C., Kangsanarak, J., \& Panyathong, R. (2010). Causes and time-course of vertigo in an ear, nose, and throat clinic. European Archives of Oto-Rhino-Laryngology : Official Journal of the European Federation of OtoRhino-Laryngological Societies (EUFOS) : Affiliated with the German Society for OtoRhino-Laryngology - Head and Neck Surgery, 267(12), 1837-1841. doi:10.1007/s00405010-1309-9; 10.1007/s00405-010-1309-9 
Iverson, G. (2007). Predicting slow recovery from sport-related concussion: The new simplecomplex distinction. Clinical Journal of Sport Medicine : Official Journal of the Canadian Academy of Sport Medicine, 17(1), 31-37. doi:10.1097/JSM.0b013e3180305e4d

Jacobson, G. P., McCaslin, D. L., Piker, E. G., Gruenwald, J., Grantham, S., \& Tegel, L. (2011). Insensitivity of the "Romberg test of standing balance on firm and compliant support surfaces" to the results of caloric and VEMP tests. Ear and Hearing, 32(6), e1-5. doi:10.1097/AUD.0b013e31822802bb

Kandel, E. R., Schwartz, J. H., \& Jessel, T. M. (2000). Principles of neural science. (4th ed., pp. 810-814). New York: McGraw-Hill.

Kandel, E. R., Schwartz, J. H., \& Jessell, T. M. (2000). The basal ganglia. In J. Butler, \& H. Lebowitz (Eds.), Principles of neural science (4th ed., pp. 853-866). New York, NY: McGraw-Hill.

Khasnis, A., \& Gokula, R. M. (2003). Romberg's test. Journal of Postgraduate Medicine, 49(2), 169-172.

Kiely, J. (2011). TBI is not just concussion. US Naval Institute Proceedings Magazine, 78-81.

Klous, M., Mikulic, P., \& Latash, M. L. (2011). Two aspects of feed-forward postural control: Anticipatory postural adjustments and anticipatory synergy adjustments. Journal of Neurophysiology, 105(5), 2275-2288. doi:10.1152/jn.00665.2010; 10.1152/jn.00665.2010

Knierim, J. (2012). Chapter 4: Basal ganglia. Neuroscience online: An electronic textbook for the neurosciences.

Kontos, A. P., Elbin, R. J., Schatz, P., Covassin, T., Henry, L., Pardini, J., \& Collins, M. W. (2012). A revised factor structure for the post-concussion symptom scale: Baseline and postconcussion factors. The American Journal of Sports Medicine, 40(10), 2375-2384. doi:10.1177/0363546512455400

Lanska, D. J., \& Goetz, C. G. (2000). Romberg's sign: Development, adoption, and adaptation in the 19th century. Neurology, (55), 1201-1206.

Lau, B. C., Collins, M. W., \& Lovell, M. R. (2011). Sensitivity and specificity of subacute computerized neurocognitive testing and symptom evaluation in predicting outcomes after sports-related concussion. The American Journal of Sports Medicine, 39(6), 1209-1216. doi:10.1177/0363546510392016

Lau, B. C., Collins, M. W., \& Lovell, M. R. (2012). Cutoff scores in neurocognitive testing and symptom clusters that predict protracted recovery from concussions in high school athletes. Neurosurgery, 70(2), 371-379. doi:10.1227/NEU.0b013e31823150f0; 10.1227/NEU.0b013e31823150f0 
Leddy, J. J., Sandhu, H., Sodhi, V., Baker, J. G., \& Willer, B. (2012). Rehabilitation of concussion and post-concussion syndrome. Sports Health, 4(2), 147-154. doi:10.1177/1941738111433673

Looney, M. A. (2006). Measurement issues in the clinical setting. In T. M. Wood, \& W. Zhu (Eds.), Measurement theory and practice in kinesiology (pp. 297-303). Champaign, IL: Human Kinetics.

Lovell, M. (2006). The ImPACT neuropsychological test battery. in R.J. echemendia (ed.), sports neuropsychology: Assessment and management of traumatic brain injury. NYC, NY: The Guilford Press.

Lovell, M. (2009). The neurophysiology and assessment of sports-related head injuries. Physical Medicine and Rehabilitation Clinics of North America, 20(1), 39-53, vii-viii. doi:10.1016/j.pmr.2008.10.003; 10.1016/j.pmr.2008.10.003

Marar, M., McIlvain, N. M., Fields, S. K., \& Comstock, R. D. (2012). Epidemiology of concussions among United States high school athletes in 20 sports. The American Journal of Sports Medicine, 40(4), 747-755. doi:10.1177/0363546511435626

Masani, K., Popovic, M. R., Nakazawa, K., Kouzaki, M., \& Nozaki, D. (2003). Importance of body sway velocity information in controlling ankle extensor activities during quiet stance. Journal of Neurophysiology, 90(6), 3774-3782. doi:10.1152/jn.00730.2002

Massion, J. (1992). Movement, posture and equilibrium: Interaction and coordination. Progress in Neurobiology, 38(1), 35-56.

McCrea, M., Barr, W. B., Guskiewicz, K., Randolph, C., Marshall, S. W., Cantu, R., ... Kelly, J. P. (2005). Standard regression-based methods for measuring recovery after sport-related concussion. Journal of the International Neuropsychological Society, 11(1), 58-69. doi:10.1017/S1355617705050083

McCrea, M., Guskiewicz, K. M., Marshall, S. W., Barr, W., Randolph, C., Cantu, R. C., . . . Kelly, J. P. (2003). Acute effects and recovery time following concussion in collegiate football players: The NCAA concussion study. The Journal of the American Medical Association, 290(19), 2556-2563. doi:10.1001/jama.290.19.2556

McCrea, M., Hammeke, T., Olsen, G., Leo, P., \& Guskiewicz, K. (2004). Unreported concussion in high school football players: Implications for prevention. Clinical Journal of Sport Medicine: Official Journal of the Canadian Academy of Sport Medicine, 14(1), 13-17.

McCrory, P., Davis, G., \& Makdissi, M. (2012). Second impact syndrome or cerebral swelling after sporting head injury. Current Sports Medicine Reports, 11(1), 21-23.

McCrory, P., Johnston, K., Meeuwisse, W., Aubry, M., Cantu, R., Dvorak, J., . . Schamasch, P. (2005). Summary and agreement statement of the second international conference on 
concussion in sport, Prague 2004. The Physician and Sportsmedicine, 33(4), 29-44.

doi:10.3810/psm.2005.04.76

McCrory, P., Meeuwisse, W., Aubry, M., Cantu, B., Dvorak, J., Echemendia, R., . . Turner, M. (2013). Consensus statement on concussion in sport-the 4th international conference on concussion in sport held in Zurich, November 2012. Physical Therapy in Sport, 14(2) doi:10.1016/j.jsams.2013.02.009; 10.1016/j.jsams.2013.02.009

McReynolds, L. V., \& Kearns, K. P. (1983). Reliability and observational codes. Single-subject experimental designs in communicative disorders (pp. 149-153). Baltimore, Maryland: University Park Press.

Menegoni, F., Tacchini, E., Bigoni, M., Vismara, L., Priano, L., Galli, M., \& Capodaglio, P. (2011). Mechanisms underlying center of pressure displacements in obese subjects during quiet stance. Journal of NeuroEngineering and Rehabilitation, 8(20), 20-0003-8-20. doi:10.1186/1743-0003-8-20; 10.1186/1743-0003-8-20

Michalski, A., Glazebrook, C. M., Martin, A. J., Wong, W. W., Kim, A. J., Moody, K. D., . . . Zabjek, K. F. (2012). Assessment of the postural control strategies used to play two Wii Fit videogames. Gait \& Posture, 36(3), 449-453. doi:10.1016/j.gaitpost.2012.04.005

Mucha, A., Collins, M., \& French, J. (2012). Augmenting neurocognitive assessment in the evaluation of sports concussion: How vestibular and ocular issues impact recovery. Brain Injury Professional, 9(1) , 12-16.

Mulligan, I., Boland, M., \& Payette, J. (2012). Prevalence of neurocognitive and balance deficits in collegiate aged football players without clinically diagnosed concussion. The Journal of Orthopaedic and Sports Physical Therapy, 42(7), 625-632. doi:10.2519/jospt.2012.3798; 10.2519/jospt.2012.3798

Murray, N. G., Pradeep Ambati, V. N., Salvatore, A. P., \& Reed-Jones, R. J. (2014). Assessment of oculomotor control and balance post-concussion: A preliminary study for a novel approach to concussion management. Brain Injury, 28(4) , 496-503. doi:10.3109/02699052.2014.887144

Nashner, L. M., Black, F. O., \& Wall, C., 3rd. (1982). Adaptation to altered support and visual conditions during stance: Patients with vestibular deficits. The Journal of Neuroscience: The Official Journal of the Society for Neuroscience, 2(5), 536-544.

NCAA sports medicine handbook. (2009-2010). Retrieved

Park, I., \& Schutz, R. W. (2006). Repeated measures and longitudinal data analysis. In T. M. Wood, \& W. Zhu (Eds.), Measurement theory and practice in kinesiology (1st ed., pp. 169197). Champaign, IL: Human Kinetics. 
Parker, T. M., Osternig, L. R., Lee, H. J., Donkelaar, P., \& Chou, L. S. (2005). The effect of divided attention on gait stability following concussion. Clinical Biomechanics (Bristol, Avon), 20(4), 389-395. doi:10.1016/j.clinbiomech.2004.12.004

Parker, T. M., Osternig, L. R., van Donkelaar, P., \& Chou, L. S. (2007). Recovery of cognitive and dynamic motor function following concussion. British Journal of Sports Medicine, 41(12), 868-73; discussion 873. doi:10.1136/bjsm.2006.033761

Peterka, R. J. (2002). Sensorimotor integration in human postural control. Journal of Neurophysiology, 88, 1097-1118.

Powers, K. C., Kalmar, J. M., \& Cinelli, M. E. (2013). Recovery of static stability following a concussion. Gait \& Posture, doi:10.1016/j.gaitpost.2013.05.026;

10.1016/j.gaitpost.2013.05.026

Prieto, T. E., Myklebust, J. B., Hoffmann, R. G., Lovett, E. G., \& Myklebust, B. M. (1996). Measures of postural steadiness: Differences between healthy young and elderly adults. IEEE Transactions on Bio-Medical Engineering, 43(9), 956-966. doi:10.1109/10.532130

Reed-Jones, R. J., Dorgo, S., Hitchings, M. K., \& Bader, J. O. (2012). WiiFit plus balance test scores for the assessment of balance and mobility in older adults. Gait \& Posture, 36(3), 430-433. doi:10.1016/j.gaitpost.2012.03.027; 10.1016/j.gaitpost.2012.03.027

Riemann, B. L., \& Guskiewicz, K. M. (2000). Effects of mild head injury on postural stability as measured through clinical balance testing. Journal of Athletic Training, 35(1), 19-25.

Riley, P. O., Mann, R. W., \& Hodge, W. A. (1990). Modelling of the biomechanics of posture and balance. Journal of Biomechanics, 23(5), 503-506.

Ruhe, A., Fejer, R., \& Walker, B. (2011). Center of pressure excursion as a measure of balance performance in patients with non-specific low back pain compared to healthy controls: A systematic review of the literature. European Spine Journal: Official Publication of the European Spine Society, the European Spinal Deformity Society, and the European Section of the Cervical Spine Research Society, 20(3), 358-368. doi:10.1007/s00586-010-1543-2; 10.1007/s00586-010-1543-2

Ryan, L. M., Warden, D. L. (2003). Post concussion syndrome. International Review of Psychiatry, 15(4), 310-316.

Salvatore, A. P., \& Fjordbak, B. S. (2011). Concussion management: The speech-language pathologist's role. Journal of Medical Speech-Language Pathology, 19(1), 1-12.

Saunders, R. L., \& Harbaugh, R. E. (1984). The second impact in catastrophic contact-sports head trauma. The Journal of the American Medical Association, 252(4), 538-539. 
Shadish, W. R., Cook, T. D., \& Campbell, D. T. (2002). Randomized experiments: Rational, designs, and conditions conducive to doing them. longitudinal designs. In K. Prancan (Ed.), Experimental and quasi-experimental designs (pp. 266-268). Boston, MA: Houghton Mifflin Company.

Shaw, N. A. (2002). The neurophysiology of concussion. Progress in Neurobiology, 67(4), 281344.

Shumway-Cook, A., \& Horak, F. B. (1986). Assessing the influence of sensory interaction of balance. suggestion from the field.66, 1548-1550.

Shumway-Cook, A., \& Horak, F. B. (2012). Normal Postural Control. In E. Lupash (Ed.), Motor control: Translating research into clinical practice (pp. 161-167). Philadelphia, PA: Lippincott Williams \& Wilkins.

Slobounov, S., Cao, C., Sebastianelli, W., Slobounov, E., \& Newell, K. (2008). Residual deficits from concussion as revealed by virtual time-to-contact measures of postural stability. Clinical Neurophysiology : Official Journal of the International Federation of Clinical Neurophysiology, 119(2), 281-289. doi:10.1016/j.clinph.2007.10.006

Slobounov, S., Gay, M., Johnson, B., \& Zhang, K. (2012). Concussion in athletics: Ongoing clinical and brain imaging research controversies. Brain Imaging and Behavior, 6(2), 224243. doi:10.1007/s11682-012-9167-2; 10.1007/s11682-012-9167-2

Slobounov, S., Slobounov, E., Sebastianelli, W., Cao, C., \& Newell, K. (2007). Differential rate of recovery in athletes after first and second concussion episodes. Neurosurgery, 61(2), 33844; discussion 344. doi:10.1227/01.NEU.0000280001.03578.FF

Slobounov, S., Tutwiler, R., Sebastianelli, W., \& Slobounov, E. (2006). Alteration of postural responses to visual field motion in mild traumatic brain injury. Neurosurgery, 59(1), 134-9; discussion 134-9. doi:10.1227/01.NEU.0000219197.33182.3F

Sparrow, W. A., \& Tirosh, O. (2005). Gait termination: A review of experimental methods and the effects of ageing and gait pathologies. Gait \& Posture, 22(4), 362-371.

Steffen, T., \& Seney, M. (2008). Test-retest reliability and minimal detectable change on balance and ambulation tests, the 36-item short-form health survey, and the unified Parkinson disease rating scale in people with Parkinsonism. Physical Therapy, 88(6), 733-746. doi:10.2522/ptj.20070214

Stewart, A. L., Hays, R. D., \& Ware, J. E. (1988). The MOS short-form general health survey: Reliability and validity in patient population. Medical Care, 26(7), 724-735.

Sullivan, L. M. (2012). Observational study designs: The cohort study. In M. Gartside (Ed.), Essentials of biostatistics in public health (pp. 9-12). Sudbury, MA: Jones \& Bartlett Learning. 
Weinstein, E., Turner, M., Kuzma, B. B., \& Feuer, H. (2013). Second impact syndrome in football: New imaging and insights into a rare and devastating condition. Journal of Neurosurgery: Pediatrics, 11(3), 331-334. doi:10.3171/2012.11.PEDS12343; 10.3171/2012.11.PEDS12343

Wikstrom, E. A. (2012). Validity and reliability of Nintendo Wii fit balance scores. Journal of Athletic Training, 47(3), 306-313. doi:10.4085/1062-6050-47.3.16

Winter, D. A. (1995). Human balance and posture control during standing and walking. Gait \& Posture, 3, 193-214.

Winter, D. A., Patla, A. E., Ishac, M., \& Gage, W. H. (2003). Motor mechanisms of balance during quiet standing. Journal of Electromyography and Kinesiology : Official Journal of the International Society of Electrophysiological Kinesiology, 13(1), 49-56.

Yamada, M., Aoyama, T., Nakamura, M., Tanaka, B., Nagai, K., Tatematsu, N., ... Ichihashi, N. (2011). The reliability and preliminary validity of game-based fall risk assessment in community-dwelling older adults. Geriatric Nursing (New York, N.Y.), 32(3), 188-194. doi:10.1016/j.gerinurse.2011.02.002; 10.1016/j.gerinurse.2011.02.002

Zatsiorsky, V. M., \& Duarte, M. (2000). Rambling and trembling in quiet standing. Motor Control, 4(2), 185-200. 


\title{
Appendix A: Informed Consent
}

\section{University of Texas at EI Paso (UTEP) Institutional Review Board Informed Consent Form for Research Involving Human Subjects}

\author{
Protocol Title: Balance and gaze assessment in concussion management \\ Principal Investigator: Anthony Salvatore, $\mathrm{PhD}$ \\ UTEP: Concussion Management Clinic
}

\begin{abstract}
In this consent form, "you" always means the study subject. If you are a legally authorized representative (such as a parent or guardian), please remember that "you" refers to the study subject.
\end{abstract}

\section{Introduction}

You are being asked to take part voluntarily in the research project described below. Please take your time making a decision and feel free to discuss it with your friends and family. Before agreeing to take part in this research study, it is important that you read the consent form that describes the study. Please ask the study researcher or the study staff to explain any words or information that you do not clearly understand.

\section{Why is this study being done?}

You have been asked to take part in a research study that provides a post-concussion management program to athletes, and others who have suffered a concussion, to help with their recovery from a concussion. Both college and high school level athletes from the EI Paso area will be participating in this program at UTEP. You are being asked to be in the study because you are an athlete.

We are excited to implement this exciting program here at UTEP. This program will assist our team physicians/athletic trainers in evaluating and treating head injuries (e.g., concussion). We will be first collecting baseline measures of cognitive and language function, auditory processing, balance, and gaze (eye movement) behavior. If you decide to enroll in this study, your involvement will be a part of this baseline assessment and will 
last approximately one hour. If at a later time a concussion is suspected, you will be required to re-take the tests. If you have already suffered an injury to your head, you will be asked to repeat these activities several times over the next month or two. Collecting both preseasons and post-injury data will allow us to examine the changes that occur due to the concussion.

\section{What is involved in the study?}

If you agree to take part in this study, the research team will collect baseline data on your balance, gaze (eye movements), language and cognitive functions. The assessments of these factors will be done using video games on a computer and Nintendo Wii system.

You will begin by taking a 15-20 minute ImPACT test. This test tracks information such as memory, reaction time, speed, and concentration. It is not an IQ test. To do this test you will sit in front of a computer monitor. There is a separate information sheet regarding this test that you will read and sign following this form.

You will then take an auditory comprehensions test. This test involves playing another game on the computer that involves a visual array of symbols that you must move around. This test will take about 5-10 minutes.

Following these computer based games you will then be asked to do some balance exercises on the Nintendo Wii system using the WiiFit Plus and balance board. During this time you will be wearing a head band that has a camera attached to it that will be monitoring the movements of your eye. We will use this to assess your gaze behavior during the games. The camera is directed at your left eye only and does not pick up any other facial information.

After the WiiFit tests, you will perform a series of clinical balance measures that involved maintaining upright stance for a short amount of time. This section should take about 5-10 minutes to complete.

You will then be assessed for a test of benign paroxysmal positional vertigo (BBPV). This test involves lying on your back while a researcher slightly manipulates your head and observes your eye reactions. This test should take about 5 minutes to complete. 
There are no known risks associated with this research

5. What will happen if I am injured in this study?

The University of Texas at El Paso and its affiliates do not offer to pay for or cover the cost of medical treatment for research related illness or injury. No funds have been set aside to pay or reimburse you in the event of such injury or illness. You will not give up any of your legal rights by signing this consent form. You should report any such injury to Anthony Salvatore at (915-747-7265) and to the UTEP Institutional Review Board (IRB) at (915-747-8841) or irb.orsp@utep.edu.

\section{Are there benefits to taking part in this study?}

There will be no direct benefits to you for taking part in this study. This research may help us to understand the nature and severity of concussion injuries. This will lead to better concussion management and prevention training for athletes, coaches, athletic trainers, administrators, parents, physicians and civic leaders.

\section{What other options are there?}

You have the option not to take part in this study. There will be no penalties involved if you choose not to take part in this study.

\section{Who is paying for this study?}

Internal Funding:

Funding for this study is provided by UTEP's graduate enhancement funds of the college of health sciences.

9. What are my costs?

There are no direct costs. You will be responsible for travel to and from the research site and any other incidental expenses.

10. Will I be paid to participate in this study? 
You are being paid to take part in this study. A $\$ 40$ gift card to either BestBuy, Target, or Applebee's will be supplied to you at the successful conclusion of this study.

\section{What if I want to withdraw, or am asked to withdraw from this study?}

Taking part in this study is voluntary. You have the right to choose not to take part in this study. If you do not take part in the study, there will be no penalty.

If you choose to take part, you have the right to stop at any time. However, we encourage you to talk to a member of the research group so that they know why you are leaving the study. If there are any new findings during the study that may affect whether you want to continue to take part, you will be told about them. The researcher may decide to stop your participation without your permission, if he or she thinks that being in the study may cause you harm.

\section{Who do I call if I have questions or problems?}

You may ask any questions you have now. If you have questions later, you may call:

Anthony P. Salvatore, Ph.D., CCC-SLP

Professor, Board Certified-ANCDS

Chair, Department of Rehabilitation Sciences

Director, Speech Language Pathology Program Director, Voice and Brain Lab

College of Health Sciences

The University of Texas at El Paso

(915)747-7239

If you have questions or concerns about your participation as a research subject, please

contact the UTEP Institutional Review Board (IRB) at (915-747-8841) or

irb.orsp@utep.edu.

\section{What about confidentiality?}

Your part in this study is confidential. None of the information will identify you by name. All records will be kept in a locked cabinet in a locked office.

\section{Authorization Statement}

I have read each page of this paper about the study (or it was read to me). I know that being in this study is voluntary and I choose to be in this study. I know I can stop being 
in this study without penalty. I will get a copy of this consent form now and can get information on results of the study later if I wish.

Participant Name:

Date:

Participant Signature:

Time:

Participant or Parent/Guardian Signature:

Consent form explained/witnessed by:

Signature

Printed name:

Date:

Time: 


\section{Appendix B: Health Status Questionnaire}

Subject \#

Health Status Questionnaire

Please complete the following questions as accurately as possible.

Date of Birth:

11

Age: yr.

Weight:

Height:

Average number of hours worked per week:
$\square$ Less than 20
$\square 20-40$
41-60
over 60

More than 25\% of time spent at work/school is: (mark all that apply)

$\begin{array}{ll}\square \text { Sitting at a desk } & \square \text { Lifting or carrying loads } \square \text { Standing } \\ \square \text { Walking } & \square \text { Driving }\end{array}$

\section{Medical History}

Please mark any who have died of heart attack before age 50 years:

$\square$ Father $\square$ Mother $\square$ Grandparent $\square$ Brother $\square$ sister

Date of last physical exam:

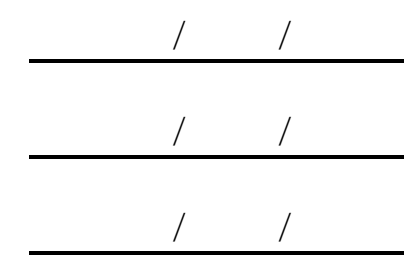

Date of last menstrual cycle:

Is there any possibility that you are pregnant? Yes

No

Please mark and date all surgeries you have had: 


\begin{tabular}{|c|c|c|c|}
\hline Back & / & $\square$ Heart & / \\
\hline Kidney & 1 & Eyes & 7 \\
\hline Joint & 1 & Neck & 7 \\
\hline Ears & 1 & Hernia & 7 \\
\hline Lung & 1 & Hysterectomy & 1 \\
\hline Other & & & 1 \\
\hline
\end{tabular}

Please mark all of the following for which you have been diagnosed or treated by a physician or health professional:

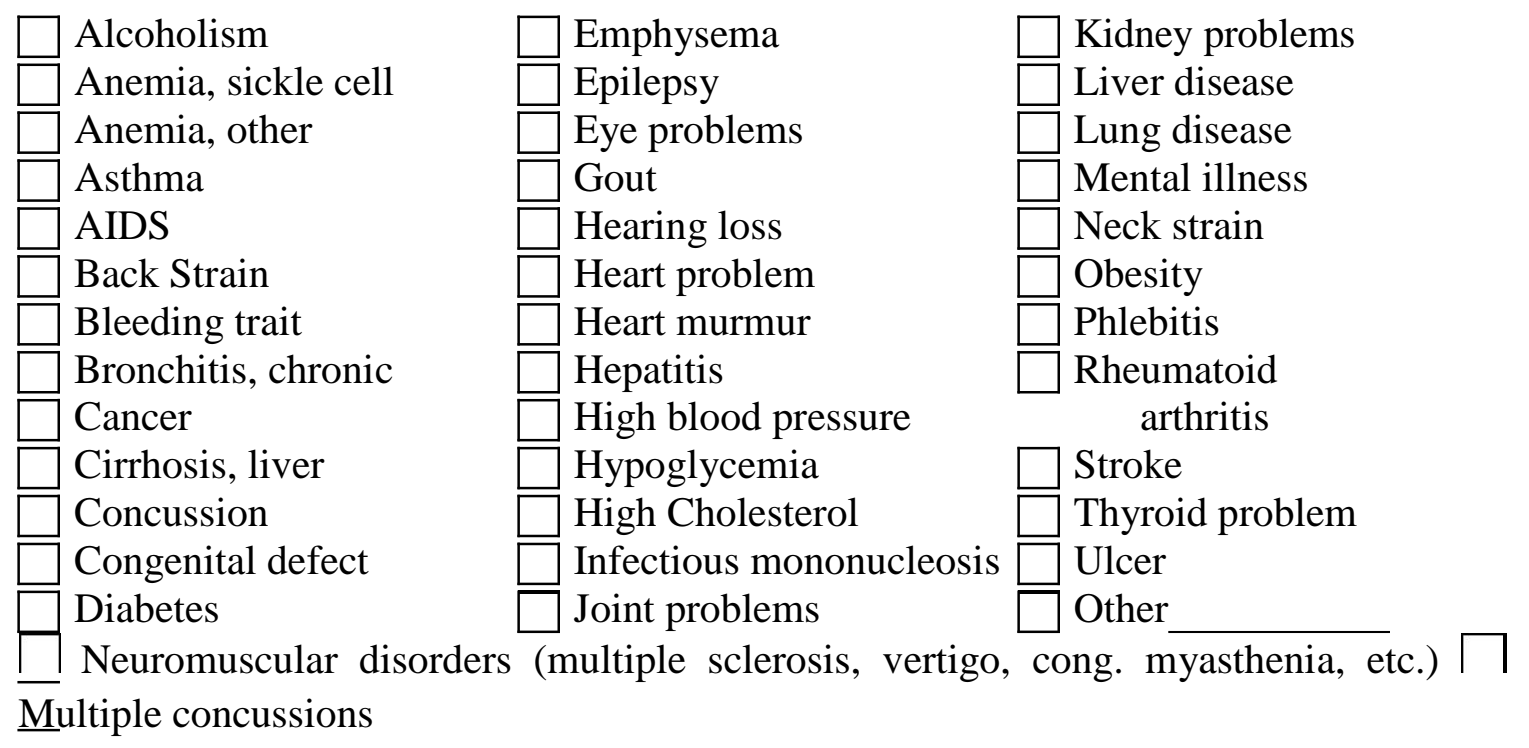

Please mark all medications/supplements taken during the past 6 months:

\begin{tabular}{lll}
$\square$ Blood thinner & $\square$ Epilepsy medication & $\square$ Other \\
Diabetic & $\square$ Heart medication & $\square$ Other \\
\hline$\square$ Diuretic & $\square$ High blood pressure medication & $\square$ Other \\
\hline Insulin & $\square$ Hormones & $\square$ Other
\end{tabular}


Please mark any of the following symptoms you have had recently:

$\square$ Abdominal pain
$\square$ Arm or shoulder pain
$\square$ Breathless with slight exertion
$\square$ Blurred vision
$\square$ Blood in urine
$\square$ Burning sensations
$\square$ Chest pain
$\square$ Cough up blood
$\square$ Difficulty walking
$\square$ Dizziness
$\square$ Feel faint

\begin{tabular}{ll}
$\square$ & Frequent urination \\
\hline$\square$ & Leg pain/numbness \\
$\square$ & Low blood sugar \\
$\square$ & Low-back pain \\
\hline$\square$ & Palpitation or fast heart beat \\
\hline$\square$ & Shortness of breath \\
\hline$\square$ & Significant emotional problem \\
$\square$ & Swollen joints \\
$\square$ & Unusual fatigue with normal \\
$\quad$ activity \\
$\square$ Weakness in arms
\end{tabular}

Have you experienced any bouts of vertigo, dizziness, or false sense of motion?

If so, how recent?

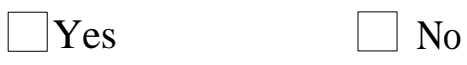

Do you ever feel lightheaded or feel the sensation of being disconnected from the physical environment?

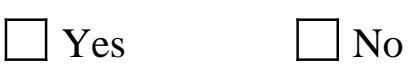

If so, how often?

Do you ever feel off-balance or wobbly while standing or walking?

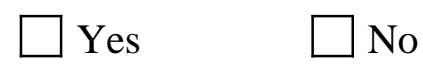

Do you or have you ever experienced motion sickness? $\quad \square$ Yes $\quad \square$ No 
Have you recently suffered from any severe head trauma/injuries (i.e. concussions, inner ear damage)

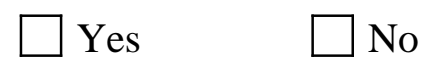

Do you experience regular migraines? $\square$ Yes $\square$ No

If so, how often and how severe?

Have you recently suffered from an ear infection of a cold? $\quad \square$ Yes $\quad \square$ No

If so, how recent? 


\section{Appendix C: History Form}

\section{Post-injury form}

\section{ALWAYS CHECK:}

- If they are under 18 the consent form must be signed by the parent/guardian

- consent forms include: observation form, confidentiality form, release of information

- pt. keeps the letter of introduction

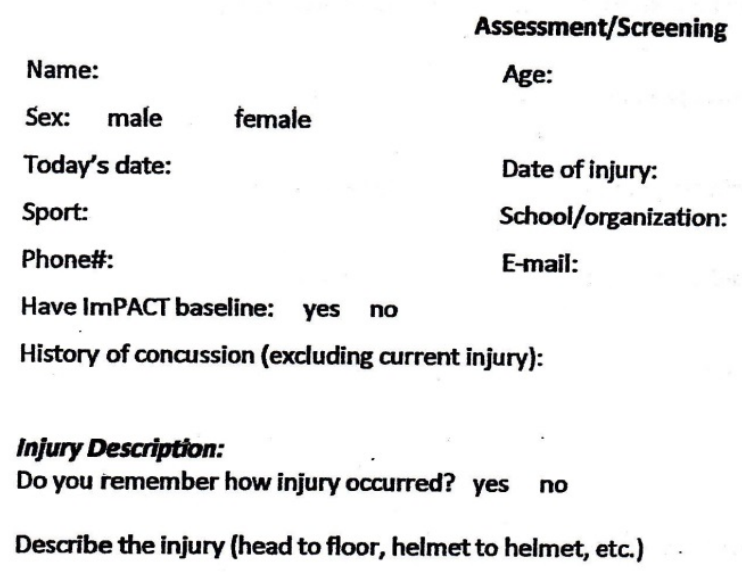

Indicate site of injury:

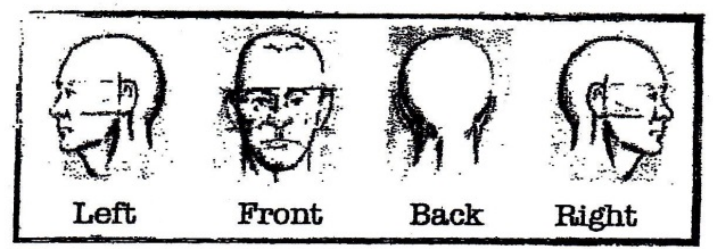

LOC? yes no

What do you remember before the injury?

What do you remember after the injury? 
Symptoms after the injury:

Ask the patient if s/he expexieristo any-of the following symptoms?

\begin{tabular}{|c|c|c|}
\hline O Headache & O Difficulty concentrating & O Nervousness \\
\hline O Dizzininéss's & O Visual problems & O Mëntälly foggy \\
\hline O Sleepiness & O Balance problems & O Nausea \\
\hline O Irritability & O Sensitivity to noise & O Fatigue \\
\hline O Emiotional & O Sensitivity to light & O Drowsiness \\
\hline O Slowed down & OVomiting & O Sadness \\
\hline
\end{tabular}

Are you having any difficulties ät school?

O Paying attention

O Difficulty concentrating

O Following instructions

O Learning new material

Last time pt. ate (diet pattern):

Romberg's Test (Balance): positive/negative

Additional notes/obsenvations during testing: 


\section{VITA}

Nicholas Gerald Murray was born and raised in Rough and Ready, California. The third child of Scott and Jennifer Murray, he graduated from Nevada Union High School, Grass Valley, California, in the spring of 2005. He entered into San Diego State University in the fall of 2005, where he graduated from San Diego State University with a bachelor's degree in Kinesiology in the spring of 2009 and a Master's degree in Kinesiology in the fall of 2010. During his graduate studies at San Diego State University, he was the assistant director for the San Diego State University Fitness Clinic for Individuals with Disabilities and taught many courses on musculoskeletal and neurological disabilities. In the summer of 2011, he entered into the Interdisciplinary Health Sciences Ph.D. Program where he studied the motor control and biomechanical deficits exhibited by individuals with concussions. While pursuing his Doctoral degree, he received a Graduate Research Associate Scholarship, published over six first author publications in international peer reviewed journals, taught courses in biomechanics, and presented his research at multiple national and international conferences.

Permanent address: 351 Thunderbird Dr. Apt 22

El Paso, Texas, 79912

This thesis/dissertation was typed by Nicholas Gerald Murray. 